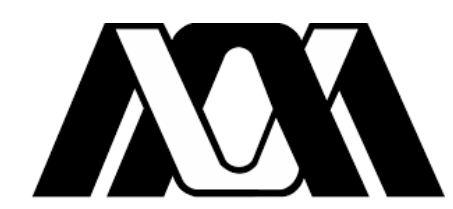

Casa abierta al tiempo
Universidad Autónoma Metropolitana

División de Ciencias Básicas e Ingeniería

Departamento de Matemáticas

Unidad Iztapalapa

\title{
ESTADOS ESTACIONARIOS DE GENERADORES DE MARKOV DEL TIPO LÍMITE DE ACOPLAMIENTO DÉBIL Y MODELOS DE TRANSPORTE CUÁNTICO
}

\author{
Tesis que presenta \\ Álvaro Hernández Cervantes \\ Para obtener el grado de \\ Doctor en Ciencias (Matemáticas)
}

\author{
Asesores: Dr. Roberto Quezada Batalla \\ Dr. Franco Fagnola
}

Jurado:

Presidente:

Dr. Octavio Arizmendi Echegaray

Secretario: Dr. Julio César García Corte

Vocal:

Dr. Franco Fagnola

Vocal:

Dr. Jorge Ricardo Bolaños Servín

Vocal:

Dr. Jesús Adrián Espínola Rocha

Ciudad de México, México

Enero 2021 



\section{Agradecimientos}

Nuestros sueños, metas y objetivos siempre van acompañados de personas especiales que nos apoyan, motivan, creen y confían en nosotros, brindándonos, de diferentes maneras, su solidaridad. Es por ello que agradezco profundamente a mi familia, quienes siempre me apoyan y me motivan a dar lo mejor de mi cada día. Agradezco infinitamente a Emilene Pliego Pliego quien ha sido y será mi motivación para seguir adelante, gracias Emi.

Quiero expresar mi más sincero agradecimiento al Dr. Roberto Quezada Batalla quien ha sido mi asesor de tesis y una persona clave en este viaje. Agradezco por sus enseñanzas, su tiempo, su dedicación, la paciencia que me tiene, la motivación de ir más allá, por todos sus muy buenos consejos y su sencillez. Es una gran persona y a quien admiro, gracias Dr. Quezada.

Quiero agradecer al Dr. Franco Fagnola, por las discusiones, comentarios, sugerencias y observaciones para mejorar y fortalecer nuestro trabajo.

Quiero agradecer al Dr. Moisés Martínez Mares, quien me ayudó a comprender un poquito del grandioso mundo de la mecánica cuántica.

Agradezco a mis sinodales por sus valiosos comentarios, sugerencias y observaciones, cuya finalidad fue hacer de este un mejor trabajo.

Agradezco a mis amigos y compañeros.

Finalmente, quiero agradecer al CONACyT, a la Universidad Autónoma Metropolitana y al Departamento de Matemáticas de la Unidad Iztapalapa, por el apoyo que me han brindado a lo largo de mis estudios doctorales. ¡Gracias!! 



\section{Índice general}

Introducción . . . . . . . . . . . . . . . . . . . . . . . . . 9

1. Preliminares 11

1.1. Semigrupos cuánticos de Markov . . . . . . . . . . . . . . . 11

1.2. Semigrupos del tipo límite de acoplamiento débil . . . . . . . . . 14

2. Estados estacionarios de generadores de Markov del tipo límite de acoplamiento débil en dimensiones bajas 19

2.1. Los estados estacionarios de generadores de Markov de WCLT tienen microcorrientes constantes en ciclos . . . . . . . . . . . 20

2.2. Estados estacionarios de generadores de Markov de WCLT en di-

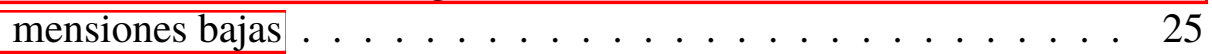

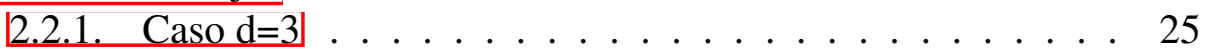

2.2 .2$. Caso $d=4 \ldots \ldots \ldots \ldots \ldots$. . . . . . . . . 26

2.3. Existencia de estados estacionarios de balance detallado local . . 28

3. Estados estacionarios de generadores de Markov de WCLT y modelos

de transporte cuántico 33

3.1. Estados estacionarios en el aniquilador de todos los operadores de

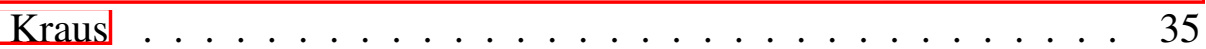

3.2. Estados estacionarios con soporte en el subespacio libre de inte-

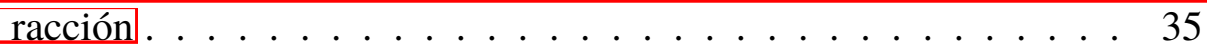

3.3. Estados estacionarios de balance detallado local . . . . . . . . . 41

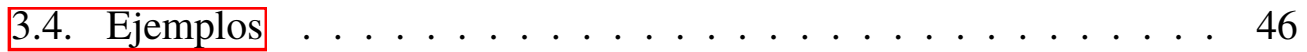

3.5. Estados estacionarios del modelo de transporte cuántico de [28] . 48

3.5.1. El modelo de transporte cuántico. . . . . . . . . . . . . 49

3.5.2. Proyecciones subarmónicas y estructura de estados esta-

3.5.3. Estados invariantes . . . . . . . . . . . . . . 53 
ÍNDICE GENERAL

3.5.4. Subespacio de recurrencia rápida . . . . . . . . . 65

3.5.5. Aproximación al equilibrio y dominios de atracción. . . . 68

Conclusiones y perspectivas . . . . . . . . . . . . 77

Apéndice A . . . . . . . . . . . . . . . . . . . 79

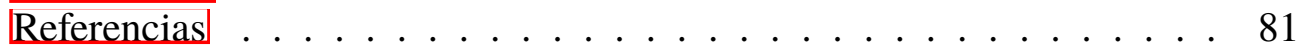




\section{Resumen}

Los semigrupos cuánticos de Markov son la herramienta matemática que permite describir la evolución de sistemas cuánticos abiertos, es decir, que interaccionan con su entorno o medio ambiente. En el presente trabajo, se estudia la estructura de los estados estacionarios de semigrupos cuánticos de Markov del tipo límite de acoplamiento débil. Más concretamente, se demuestra que cualquier estado estacionario se escribe como una combinación convexa de dos estados, el primero con soporte en el subespacio libre de interacción $W_{D}$ y el segundo soportado en el complemento ortogonal del primero. Además, como aplicación de los resultados previos, se describe en detalle la estructura de los estados estacionarios de un modelo de transporte cuántico motivado por el trabajo de Aref'eva, Kozyrev y Volovich [6], en términos de operadores de aniquilación y creación generalizados. Este modelo se estudió por primera vez en [28].

Palabras Claves: Semigrupos cuánticos de Markov, Límite de acoplamiento débil, Estados estacionarios, Generadores de Markov, Transporte cuántico. 



\section{Introducción}

Los Semigrupos Cuánticos de Markov (QMS por sus siglas en inglés) -conocidos también como Semigrupos Dinámicos Cuánticos- son la herramienta fundamental para el modelado matemático de sistemas cuánticos abiertos que interactúan con entornos externos (medio ambiente). El creciente interés en fenómenos como la decoherencia [12, 17], ruido blanco e información cuántica [39], computación cuántica y control [2], convergencia al equilibrio [10, 15, 21, 27], producción de entropía [13, 23, 24, 32, 33], entre otros, motivan la investigación sobre los QMS y sus propiedades. En 1976, Gorini, Kossakowski, Sudharshan [31] y Lindblad [38] caracterizaron la estructura del generador infinitesimal de un semigrupo cuántico de Markov uniformemente continuo. Sin embargo, esta estructura es bastante general y necesita una mayor especialización si se requiere estudiar ciertos fenómenos y/o problemas físicos concretos como el transporte cuántico.

Los semigrupos cuánticos de Markov del tipo límite de acoplamiento débil (QMS-WCLT por sus siglas en inglés) (ver [1]), son una clase especial de QMS uniformemente continuos, para ser más precisos, son semigrupos de transformaciones completamente positivas, estrechamente relacionados a un Hamiltoniano de referencia $H$ con espectro discreto y notables propiedades estructurales. Los generadores de estos semigrupos se escriben como una suma de otros generadores, uno para cada frecuencia de Bohr. Su estructura es lo suficientemente simple que permite el cálculo explícito de sus estados estacionarios (invariantes), pero lo suficientemente rica como para mostrar estados de balance detallado (estados de equilibrio), así como estados invariantes sin balance detallado pero de equilibrio local.

En [1], los autores introducen la estructura de generadores de Markov del tipo límite de acoplamiento débil mediante técnicas de límite estocástico y comienzan a estudiar la estructura de los estados estacionarios de estos generadores, clasifi- 
Introducción

cando subclases especiales de estados estacionarios con propiedades que sean lo suficientemente ricas como para ir más allá de la situación de equilibrio.

Una caracterización de esta clase de semigrupos con Hamiltoniano de referencia no degenerado, en términos de un álgebra maximal puramente atómica y ciertos subespacios de operadores asociados de manera natural e invariantes bajo la acción del semigrupo, se realizó en [20]. Por otra parte, en [17] se caracteriza la estructura de los estados estacionarios de semigrupos cuánticos de Markov uniformemente continuos con una subálgebra atómica libre de decoherencia y un estado invariante fiel.

En [9], se caracteriza la estructura de los estados invariantes de semigrupos cuánticos de Markov del tipo límite de acoplamiento débil cuando estos admiten una única frecuencia de Bohr.

En [28], los autores estudian un modelo de transporte cuántico motivado por el trabajo de Aref'eva, Kozyrev y Volovich [6]. En particular, caracterizan los estados estacionarios que pertenecen al conmutante del Hamiltoniano $\{H\}^{\prime}$.

Con esta motivación, el enfoque del presente trabajo es estudiar la estructura de todos los estados estacionarios de semigrupos cuánticos de Markov del tipo límite de acoplamiento débil, propiedades específicas y relevantes de los mismos, por ejemplo, distinguir los estados estacionarios extremos. Finalmente, aplicar los resultados previos para estudiar los estados estacionarios del modelo de transporte cuántico estudiado en [28].

El contenido del presente trabajo se ha organizado de la manera siguiente. El capítulo 1 contiene los preliminares de la teoría de semigrupos cuánticos de Markov uniformemente continuos y del tipo límite de acoplamiento débil. El capítulo 2 está dedicado al estudio de los estados estacionarios de generadores de Markov del tipo límite de acoplamiento débil en dimensiones bajas y existencia de estados estacionarios de balance detallado local. En el capítulo 3 se describe la estructura de los estados estacionarios de generadores de Markov del tipo límite de acoplamiento débil. En particular, se describe detalladamente la estructura de los estados estacionarios del modelo de transporte cuántico estudiado en [28], en términos de operadores de aniquilación y creación generalizados. Finalmente, se presentan las conclusiones y perspectivas del trabajo. 


\section{Capítulo 1}

\section{Preliminares}

En el presente capítulo se estudian los elementos necesarios sobre la teoría de semigrupos cuánticos de Markov uniformemente continuos y del tipo límite de acoplamiento débil, la estructura de sus generadores y otras herramientas necesarias para el desarrollo de este trabajo.

\subsection{Semigrupos cuánticos de Markov}

Sea $\mathrm{h}$ un espacio de Hilbert complejo separable con producto interno $\langle\cdot, \cdot\rangle$. $\mathcal{B}(\mathrm{h})$ denota el álgebra de von Neumann de operadores lineales acotados actuando sobre $\mathrm{h} ;\left(L_{1}(\mathrm{~h}),\|\rho\|_{1}=\operatorname{tr}|\rho|\right)$ denota el espacio de Banach de operadores lineales acotados actuando sobre $h$ cuya traza es finita.

Definición 1.1. Una transformación lineal acotada $T_{t}: \mathcal{B}(\mathrm{h}) \rightarrow \mathcal{B}(\mathrm{h})$ es completamente positiva, para cada $t \geq 0$ si y sólo si para cualquier par de sucesiones finitas $\left\{x_{i}\right\}_{i=1}^{n},\left\{y_{j}\right\}_{j=1}^{n} \in \mathcal{B}(\mathrm{h})$,

$$
\sum_{i, j=1}^{n} y_{i}^{*} T_{t}\left(x_{i}^{*} x_{j}\right) y_{j} \geq 0 .
$$

Definición 1.2. Un semigrupo cuántico de Markov uniformemente continuo sobre $\mathcal{B}(\mathrm{h})$, es una familia $\left\{T_{t}\right\}_{t \geq 0}$ de transformaciones (operadores) lineales acotadas actuando sobre $\mathcal{B}(\mathrm{h})$ que satisface las propiedades siguientes:

- $T_{0}=I$.

- $T_{s+t}=T_{s} T_{t}$, para todo $s, t \geq 0$. (Propiedad de Semigrupo) 
- $\lim _{t \rightarrow 0^{+}}\left\|T_{t}-I\right\|_{\mathcal{B}(\mathcal{B}(\mathrm{h}))}=0$. (Continuidad Uniforme)

- $T_{t}(\mathbb{1})=\mathbb{1}$, para todo $t \geq 0$. (Propiedad de Markov o Conservatividad $)$

- $T_{t}$ es una transformación completamente positiva, para cada $t \geq 0$.

- $T_{t}$ es una transformación continua con respecto a la topología $\sigma$-débil sobre $\mathcal{B}(\mathrm{h})$, para cada $t \geq 0$.

Definición 1.3. El operador lineal $\mathcal{L}: \mathcal{D}(\mathcal{L}) \subset \mathcal{B}(\mathrm{h}) \rightarrow \mathcal{B}(\mathrm{h})$ definido por:

$$
\mathcal{D}(\mathcal{L}):=\left\{x \in \mathcal{B}(\mathrm{h}): \lim _{t \rightarrow 0^{+}} \frac{T_{t}(x)-x}{t} \text { existe }\right\}
$$

$y$

$$
\mathcal{L}(x)=\lim _{t \rightarrow 0^{+}} \frac{T_{t}(x)-x}{t}=\left.\frac{d^{+} T_{t}(x)}{d t}\right|_{t=0}
$$

para $x \in \mathcal{D}(\mathcal{L})$, es el generador infinitesimal del semigrupo $\left(T_{t}, 0 \leq t<\infty\right)$, $\mathcal{D}(\mathcal{L})$ es el dominio de $\mathcal{L}$ y un subespacio vectorial de $\mathcal{B}(\mathrm{h})$.

Teorema 1.1. Un operador $\mathcal{L}$ es el generador infinitesimal de un semigrupo uniformemente continuo si y sólo si $\mathcal{L}$ es un operador lineal acotado (ver [30 41]]).

Observación 1.1. Si $\mathcal{L}$ es el generador infinitesimal de un semigrupo uniformemente continuo, el semigrupo $\left\{T_{t}\right\}_{t \geq 0}$ tiene la expresión explícita

$$
T_{t}=\sum_{n=0}^{\infty} \frac{t^{n}}{n !} \mathcal{L}^{n},
$$

donde para cada $t$, la serie converge en la norma de operadores sobre $\mathcal{B}(\mathrm{h})$.

Ejemplo 1.1. Sea $H$ un operador autoadjunto en $\mathcal{B}(\mathrm{h})$. El semigrupo $\left\{T_{t}\right\}_{t \in \mathbb{R}}$ asociado al grupo unitario uniparamétrico $\left\{e^{i t H}\right\}_{t \in \mathbb{R}} y$ definido mediante $T_{t}(x)=$ $e^{-i t H} x e^{i t H}$, es un $Q M S$ uniformemente continuo con generador infinitesimal $\mathcal{L}$ definido como:

$$
\mathcal{L}(x)=-i H x+i x H=-i[H, x], \quad \text { para cada } x \in \mathcal{B}(\mathrm{h}),
$$

donde $[\cdot, \cdot]$ denota al conmutador, definido como $[x, y]:=x y-y x$, para todo $x, y \in \mathcal{B}(\mathrm{h})$. 
Más ejemplos de QMS uniformemente continuos pueden encontrarse en los capítulos 2 y 3 del presente trabajo, y en [14, 19]. Además, en [3] se encuentran ejemplos provenientes de ciertos modelos fenomenológicos de óptica cuántica, estudiados a partir de técnicas del límite estocástico. El resultado siguiente es una caracterización del generador infinitesimal de un semigrupo cuántico de Markov uniformemente continuo (ver [31, 35, 38]).

Teorema 1.2. Un operador lineal acotado $\mathcal{L}$ actuando sobre $\mathcal{B}(\mathrm{h})$ es el generador infinitesimal de un semigrupo cuántico de Markov uniformemente continuo si y sólo si existe un operador completamente positivo $\Phi$ definido sobre $\mathcal{B}(\mathrm{h})$ y un operador $G \in \mathcal{B}(\mathrm{h})$ tal que:

- $\mathcal{L}(x)=\Phi(x)-G^{*} x-x G, \quad$ para todo $x \in \mathcal{B}(\mathrm{h})$.

- $G+G^{*} \leq \mathcal{L}(\mathbb{1})$.

Al generador infinitesimal de un semigrupo cuántico de Markov uniformemente continuo se le conoce como generador GKSL, por sus creadores Gorini, Kossakowski, Sudarshan [31] y Lindblad [38].

Definición 1.4. Un estado $\varrho$ sobre $\mathcal{B}(\mathrm{h})$ es un funcional lineal continuo, positivo y de norma uno. Además, @ es normal si y sólo si $\left(\varrho\left(a_{\alpha}\right)\right)_{\alpha}$ converge a $\varrho(a)$, para cada red creciente de operadores $\left(a_{\alpha}\right)_{\alpha}$ con supremo a.

Observación 1.2. Cada estado normal se identifica con un operador $\rho$ definido sobre h, positivo y de traza uno, llamado densidad en analogía con las funciones de densidad en probabilidad clásica, que satisface $\varrho(x)=\operatorname{tr}(\rho x)$ para todo $x \in$ $\mathcal{B}(\mathrm{h})$. Por esta razón, denominaremos estados a los operadores $\rho$ sobre h, positivos $y$ de traza uno (ver [44]).

Definición 1.5. Sea $\rho$ un estado, se dice que $\rho$ es fiel si $\operatorname{ker} \rho=\{0\}$.

Definición 1.6. $\mathcal{B}(\mathrm{h})_{*}$ es el espacio de funcionales lineales $\sigma$-débilmente continuos sobre $\mathcal{B}(\mathrm{h})$ y se llama espacio predual de $\mathcal{B}(\mathrm{h})$.

Observación 1.3. El teorema de Schatten [42] establece la existencia de dos isomorfismos isométricos, el primero entre $\mathcal{B}(\mathrm{h})$ y $L_{1}(\mathrm{~h})^{*}$ y el otro entre $\mathcal{B}(\mathrm{h})_{*} y$ $L_{1}(\mathrm{~h})$, por tal motivo, para cada $t$, al operador $T_{t}: \mathcal{B}(\mathrm{h}) \mapsto \mathcal{B}(\mathrm{h})$ le corresponde un único operador $T_{* t}: \mathcal{B}(\mathrm{h})_{*} \mapsto \mathcal{B}(\mathrm{h})_{*}$ definido mediante la relación de dualidad

$$
\operatorname{tr}\left(x T_{* t}(\sigma)\right)=\operatorname{tr}\left(T_{t}(x) \sigma\right), \quad x \in \mathcal{B}(\mathrm{h}), \quad \sigma \in L_{1}(\mathrm{~h}) .
$$


Con base en lo anterior, tenemos que $\left\{T_{* t}\right\}_{t \geq 0}$ es a su vez un QMS uniformemente continuo sobre $L_{1}(\mathrm{~h})$, llamado semigrupo predual asociado a $\left\{T_{t}\right\}_{t \geq 0}$. $\mathcal{L}_{*}$ es el generador del semigrupo predual y la relación entre éste y $\mathcal{L}$, el generador del semigrupo directo, está dada por la relación de dualidad

$$
\operatorname{tr}\left(x \mathcal{L}_{*}(\sigma)\right)=\operatorname{tr}(\mathcal{L}(x) \sigma), \quad x \in \mathcal{B}(\mathrm{h}), \quad \sigma \in L_{1}(\mathrm{~h}) .
$$

Definición 1.7. Un estado $\rho$ se dice estacionario (invariante) respecto a un $Q M S$ $\left\{T_{t}\right\}_{t \geq 0}$ con generador predual asociado $\mathcal{L}_{*}$ si y sólo si $\mathcal{L}_{*}(\rho)=0$.

Definición 1.8. Un estado estacionario $\rho$ es extremo si no puede escribirse como una combinación convexa de dos estados estacionarios, es decir, si no puede escribirse como $\rho=\lambda \rho_{1}+(1-\lambda) \rho_{2}$, con $\rho_{1}, \rho_{2}$ estados estacionarios y $0<\lambda<1$.

Definición 1.9. Un observable $x \in \mathcal{B}(\mathrm{h})$ es un punto fijo de un $Q M S\left\{T_{t}\right\}_{t \geq 0}$ si $y$ sólo si $\mathcal{L}(x)=0$.

Definición 1.10. Sea $\mathcal{M}$ un subconjunto no vacío de $\mathcal{B}(\mathrm{h})$. Se define el conmutante de $\mathcal{M}$ por $\mathcal{M}^{\prime}:=\{x \in \mathcal{B}(\mathrm{h}): m x=x m$ para todo $m \in \mathcal{M}\}$. El doble conmutante $\mathcal{M}^{\prime \prime}$ de $\mathcal{M}$ se define de forma recursiva como $\mathcal{M}^{\prime \prime}:=\left(\mathcal{M}^{\prime}\right)^{\prime}$.

\subsection{Semigrupos del tipo límite de acoplamiento débil}

Consideremos generadores de Markov del tipo límite de acoplamiento débil $\mathcal{L}$, asociados con un operador positivo autoadjunto $H$ (llamado Hamiltoniano de referencia), con descomposición espectral discreta

$$
H=\sum_{\varepsilon_{m} \in \operatorname{Sp}(H)} \varepsilon_{m} P_{\varepsilon_{m}}
$$

donde $\varepsilon_{m}$ son los valores propios y $P_{\varepsilon_{m}}$ es la proyección espectral sobre el subespacio propio asociado a $\varepsilon_{m}$.

El subconjunto de pares ordenados de valores propios

$$
B_{+}:=\left\{\left(\varepsilon_{n}, \varepsilon_{m}\right): \omega:=\varepsilon_{n}-\varepsilon_{m}>0\right\}
$$

es el conjunto de frecuencias de Bohr y

$$
B_{+, \omega}:=\left\{\left(\varepsilon_{n}, \varepsilon_{m}\right) \in B_{+}: \varepsilon_{n}-\varepsilon_{m}=\omega\right\}
$$


es el conjunto de pares de valores propios asociados con la frecuencia $\omega$.

Un generador de Markov del tipo límite de acoplamiento débil (abreviado por generador de Markov de WCLT) tiene la estructura

$$
\mathcal{L}=\sum_{\omega \in B_{+}} \mathcal{L}_{\omega}
$$

donde para cada frecuencia de Bohr $\omega$ y para cada $x \in \mathcal{B}(\mathrm{h})$ el generador directo $\mathcal{L}_{\omega}$ tiene la estructura canónica de Gorini-Kossakowski-Sudarchan-Lindbland (estructura GKSL), definida mediante

$$
\begin{aligned}
\mathcal{L}_{\omega}(x): & =i\left[\Delta_{\omega}, x\right] \\
& -\Gamma_{-, \omega}\left(\frac{1}{2}\left\{D_{\omega}^{*} D_{\omega}, x\right\}-D_{\omega}^{*} x D_{\omega}\right)-\Gamma_{+, \omega}\left(\frac{1}{2}\left\{D_{\omega} D_{\omega}^{*}, x\right\}-D_{\omega} x D_{\omega}^{*}\right)
\end{aligned}
$$

con operadores de interacción parcial (u operadores de Kraus) $D_{\omega}$ definidos como

$$
D_{\omega}:=\sum_{\left(\varepsilon_{n}, \varepsilon_{m}\right) \in B_{+, \omega}} P_{\varepsilon_{m}} D P_{\varepsilon_{n}}
$$

donde $D \in \mathcal{B}(\mathrm{h})$ y es llamado operador de interacción. El Hamiltoniano efectivo es el operador $\Delta_{\omega}$ que satisface

$$
\Delta_{\omega}=\Delta_{\omega}^{*} \in\{H\}^{\prime}
$$

donde $\{H\}^{\prime}$ es el conmutante de $H$ y los coeficientes se definen de la misma forma que en [1], es decir, por

$$
\Gamma_{+, \omega}:=b_{\omega} \frac{1}{e^{\beta(\omega) \omega}-1} ; \quad \Gamma_{-, \omega}:=b_{\omega} \frac{e^{\beta(\omega) \omega}}{e^{\beta(\omega) \omega}-1} ; \quad b_{\omega} \geq 0
$$

donde $\beta$ es una función suficientemente regular (temperatura inversa) con

$$
\beta(\omega)>0 ; \quad \forall \omega \in B_{+} .
$$

El generador de Markov de WCLT predual tiene la forma

$$
\mathcal{L}_{*}=\mathcal{L}_{* 0}-i \delta_{\Delta} \sqrt{1}
$$

\footnotetext{
${ }^{1} \mathcal{L}_{* 0}$ se conoce como la parte disipativa y $\delta_{\Delta}$ se conoce como la parte hamiltoniana.
} 
con

$$
\begin{aligned}
\mathcal{L}_{* 0}(\rho) & =\sum_{\omega \in B_{+}}\left(-\frac{\Gamma_{-, \omega}}{2}\left\{D_{\omega}^{*} D_{\omega}, \rho\right\}+\Gamma_{+, \omega} D_{\omega}^{*} \rho D_{\omega}\right) \\
& +\left(-\frac{\Gamma_{+, \omega}}{2}\left\{D_{\omega} D_{\omega}^{*}, \rho\right\}+\Gamma_{-, \omega} D_{\omega} \rho D_{\omega}^{*}\right)
\end{aligned}
$$

$\mathrm{y}$

$$
\delta_{\Delta}(\rho)=\sum_{\omega \in B_{+}} \delta_{\Delta_{\omega}}(\rho), \quad \delta_{\Delta_{\omega}}(\rho)=\left[\Delta_{\omega}, \rho\right] .
$$

En el caso cuando el conjunto de las frecuencias de Bohr es infinito, para que $\mathcal{L}$ sea el generador de un QMS uniformemente continuo, la serie

$$
\sum_{\omega \in B_{+}}\left(\Gamma_{-\omega} D_{\omega}^{*} D_{\omega}+\Gamma_{+\omega} D_{\omega} D_{\omega}^{*}\right)
$$

debe ser fuertemente convergente en $\mathcal{B}(\mathrm{h})$ (ver corolario 30.13 p. 268 y teorema 30.16 p. 271 en [40]).

Definición 1.11. Sea $\mathcal{L}$ un generador de Markov, se dice que $\mathcal{L}$ es genérico si

(1) el Hamiltoniano de referencia $H$ es no degenerado (equivalentemente, todas las proyecciones ortogonales en la descomposición espectral de $H$ son de rango uno).

(2) para cada frecuencia de Bohr $\omega$ existe un único par de valores propios $\left(\epsilon_{n}, \epsilon_{m}\right), m<n$ tal que $\omega=\epsilon_{n}-\epsilon_{m}$.

Consideraciones 1. a) A partir de ahora vamos a escribir simplemente $P_{n}$ en lugar de $P_{\varepsilon_{n}}$ siempre que no exista confusión.

b) Dado un estado $\rho$ y un subespacio $V$, " $\rho$ está soportado en $V$ ” significa que "el soporte de $\rho$ es un subespacio de $V$ ".

c) Sea $T \in \mathcal{B}(\mathrm{h})$ un operador arbitrario, el soporte de $T$ (denotado por supp $T$ ) se define como "la cerradura de su rango", es decir, supp $T=\overline{\operatorname{Ran} T}$.

d) Denotaremos por $\left\{e_{n}\right\}_{n \geq 0}$ una base ortonormal de $\mathrm{h}$ de vectores propios de $H$ (i.e. una base ortonormal del Hamiltoniano de referencia $H$ ), siempre que no exista confusión. 
e) Consideraremos $\mathrm{h}=\mathbb{C}^{d}$ cuando el espacio de Hilbert $\mathrm{h}$ sea de dimensión finita. En este caso, $\mathcal{B}\left(\mathbb{C}^{d}\right)=\mathcal{M}_{d \times d}(\mathbb{C})$ es el álgebra de matrices cuadradas con entradas complejas.

Ahora poseemos los elementos necesarios para comenzar el estudio de la estructura de los estados estacionarios de generadores de Markov del tipo límite de acoplamiento débil. El capítulo siguiente esta dedicado al estudio de los estados estacionarios en dimensiones bajas. 



\section{Capítulo 2}

\section{Estados estacionarios de generadores de Markov del tipo límite de acoplamiento débil en dimensiones bajas}

En este capítulo se presentan algunos resultados acerca de la estructura de estados estacionarios de generadores de Markov del tipo límite de acoplamiento débil en dimensiones bajas, y la existencia de estados estacionarios que satisfacen la condición de balance detallado local. En la sección 2.1] se estudian las microcorrientes en los estados estacionarios de QMS-WCLT. En la sección 2.2, se exhibe la estructura explícita de estados estacionarios de QMS-WCLT en dimensiones bajas. Finalmente, en la sección 2.3 se demuestra la existencia de estados estacionarios que satisfacen una condición de equilibrio local, pero que no satisfacen la condición de balance detallado. A continuación, se enuncian algunas consideraciones necesarias para el desarrollo del capítulo, mismas que pueden consultarse en [1] .

Consideraciones 2. a) $V_{\mathcal{L}}:=\operatorname{Sp}(H)^{1} y E_{\mathcal{L}}:=\left.\bigcup_{\left\{\omega \in B_{+}: \Gamma_{ \pm, \omega}>0\right\}} B_{+, \omega}\right|^{2}$

b) Un estado $\rho \in\{H\}^{\prime \prime}$, satisface la condición de balance detallado (equilibrio) si las ecuaciones siguientes se satisfacen:

$$
\rho_{\epsilon_{m}} \Gamma_{+, \omega}=\rho_{\epsilon_{n}} \Gamma_{-, \omega} ; \quad \forall \omega \in B_{+}, \forall\left(\epsilon_{n}, \epsilon_{m}\right) \in B_{+, \omega} .
$$

\footnotetext{
${ }^{1} \mathrm{Sp}(H)$ denota el espectro del Hamiltoniano de referencia $H$.

${ }^{2} B_{+, \omega}$ denota el conjunto de aristas (con salida en $\epsilon_{n}$ y llegada en $\epsilon_{m}$ ) de longitud $\omega$.
} 
c) Un estado $\rho \in\{H\}^{\prime \prime}$, satisface la condición de balance detallado local si se satisfacen algunas de las ecuaciones anteriores, pero no todas.

\subsection{Los estados estacionarios de generadores de Mar- kov de WCLT tienen microcorrientes constantes en ciclos}

El caso cuando $H$ tiene espectro no degenerado se caracteriza por la condición $\{H\}^{\prime}=\{H\}^{\prime \prime}$ o equivalentemente, todas las proyecciones ortogonales en la descomposición espectral de $H$ son proyecciones de rango uno. En este caso, la proposición siguiente fue demostrada en [1].

Proposición 2.1. Con las notaciones e hipótesis del teorema 4.1 en [I]], sea H un Hamiltoniano de referencia no degenerado. Si

$$
\rho=\sum_{\epsilon_{m} \in \operatorname{Sp}(H)} \rho_{\epsilon_{m}}\left|e_{m}\right\rangle\left\langle e_{m}\right|
$$

entonces,

$$
\mathcal{L}_{\omega *}(\rho)=\sum_{\left(\epsilon_{n}, \epsilon_{m}\right) \in \hat{B}_{+, \omega}}\left(\rho_{\epsilon_{m}} \Gamma_{+, \omega}-\rho_{\epsilon_{n}} \Gamma_{-, \omega}\right)\left|\left\langle e_{m}, D e_{n}\right\rangle\right|^{2}\left(\left|e_{n}\right\rangle\left\langle e_{n}|-| e_{m}\right\rangle\left\langle e_{m}\right|\right) .
$$

Algunos de los resultados siguientes fueron demostrados en [1].

Teorema 2.1. Asuma que h tiene dimensión finita. Sea $H$ un Hamiltoniano de referencia no degenerado y $\rho=\sum_{\epsilon_{n} \in S p(H)} \rho_{\epsilon_{n}}\left|e_{n}\right\rangle\left\langle e_{n}\right|$ una función del Hamiltoniano.

Si $\mathcal{L}$ es un generador de Markov de WCLT asociado con H, entonces

(i) La ecuación $\mathcal{L}_{*}(\rho)=0$ tiene la forma

$$
\mathcal{Q} \mathbb{V}=0,
$$

donde $\mathcal{Q}$ es la matriz de incidencia de la correspondiente gráfica de interacción $\mathcal{G}(\mathcal{L})($ ver $[\mathbb{Z}])$ y $\mathbb{J}=\left(J_{\epsilon_{m_{j}} \epsilon_{j}}^{\left(\omega_{i}\right)}\right)_{1 \leq i \leq d, 1 \leq j \leq l_{i}}$ es el vector de corriente (o simplemente corriente) que satisface el sistema $\Gamma \rho=\sqrt{3}$ explícitamente,

$$
J_{\epsilon_{m_{j}} \epsilon_{n_{j}}}^{\left(\omega_{i}\right)}=\left(\rho_{\epsilon_{m_{j}}} \Gamma_{+, \omega_{i}}-\rho_{\epsilon_{n_{j}}} \Gamma_{-, \omega_{i}}\right)\left|\left\langle e_{m_{j}}, D e_{n_{j}}\right\rangle\right|^{2},
$$

\footnotetext{
${ }^{3} \Gamma$ es una matriz que tiene como entradas los coeficientes o tasas de transición $\Gamma_{ \pm, \omega}$ 's.
} 
para $\left(\epsilon_{n_{j}}, \epsilon_{m_{j}}\right) \in B_{+, \omega_{i}}$. Por lo tanto, cualquier solución positiva y de traza

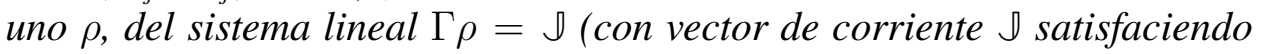
(2.2) en el rango de la matriz $\Gamma$ del sistema $\Gamma \rho=\sqrt{ })$ ), es un estado invariante.

(ii) La ecuación (2.2) tiene como máximo $\left|E_{\mathcal{L}}\right|-\left|V_{\mathcal{L}}\right|+k$ soluciones linealmente independientes, donde $k$ es el número de componentes conexas de la correspondiente gráfica de interacción $\mathcal{G}(\mathcal{L})$ (ver [1]]). Además, cada ciclo $c=\left(\epsilon_{n_{j}}\right)_{1 \leq j \leq l}$ de $\mathcal{G}$ tiene asociada una solución $\mathbb{J}_{c}$ de (2.2), siempre que el operador de interacción no se anule en los vértices del ciclo, es decir, $\left|\left\langle e_{m_{j}}, D e_{n_{j}}\right\rangle\right|^{2} \neq 0, \quad \forall 0 \leq j \leq l$.

(iii) Bajo las hipótesis del inciso (i), la condición

$$
\left|E_{\mathcal{L}}\right|-\left|V_{\mathcal{L}}\right|+k \geq 0
$$

se satisface. Si $\left|V_{\mathcal{L}}\right|=\left|E_{\mathcal{L}}\right|+k$, cualquier estado de la clase descrita en $(i)$ satisface una condición de balance detallado.

Demostración. (i) y (iii) se probaron en [1]. Para probar (ii), sea $\mathcal{Q}$ la matriz de incidencia de la correspondiente gráfica de interacción $\mathcal{G}(\mathcal{L})$. Por el teorema del rango-nulidad, se tiene que $\operatorname{rango}(\mathcal{Q})+\operatorname{nulidad}(\mathcal{Q})=\left|E_{\mathcal{L}}\right|$. Basta demostrar que cada ciclo $c=\left(\epsilon_{n_{j}}\right)_{1 \leq j \leq l}$ en la gráfica de interacción $\mathcal{G}$ tal que $\left|\left\langle e_{m_{j}}, D e_{n_{j}}\right\rangle\right|^{2} \neq$ $0, \quad \forall 0 \leq j \leq l$, corresponde a un elemento en el $\operatorname{ker}(\mathcal{Q})$. En efecto, sea $\mathbb{J}_{c}=\left(J_{\epsilon_{m_{j}} \epsilon_{n_{j}}}^{\left(\omega_{i}\right)}\right)_{1 \leq i \leq d, 1 \leq j \leq l_{i}}$ el vector de corriente asociado con $c$ definido de la manera siguiente: $J_{\epsilon_{m_{j}} \epsilon_{n_{j}}}^{\left(\omega_{i}\right)}=0$ si la arista $\left(\epsilon_{n_{j}}, \epsilon_{m_{j}}\right) \notin E_{c} ; J_{\epsilon_{m_{j}} \epsilon_{n_{j}}}^{\left(\omega_{i}\right)}=1$ si la arista $\left(\epsilon_{n_{j}}, \epsilon_{m_{j}}\right) \in E_{c}$ y tienen la misma orientación; y $J_{\epsilon_{m_{j}} \epsilon_{n_{j}}}^{\left(\omega_{i}\right)}=-1$ si la arista $\left(\epsilon_{n_{j}}, \epsilon_{m_{j}}\right) \in E_{c}$ y las orientaciones son opuestas (se puede elegir $\mathbb{J}_{c}$ de esta manera, puesto que $\left|\left\langle e_{m_{j}}, D e_{n_{j}}\right\rangle\right|^{2} \neq 0, \forall 0 \leq j \leq l$ ). Por lo tanto,

$$
\sum_{\left(\epsilon_{n_{j}}, \epsilon_{m_{j}}\right) \in \hat{B}_{+}\left(\omega_{i}\right) ; 1 \leq i \leq d} J_{\epsilon_{m_{j}} \epsilon_{n_{j}}}^{\left(\omega_{i}\right)}\left(e_{n_{j}}-e_{m_{j}}\right)=\sum_{\left(\epsilon_{n_{j}}, \epsilon_{m_{j}}\right) \in E_{c}} J_{\epsilon_{m_{j}} \epsilon_{n_{j}}}^{\left(\omega_{i}\right)}\left(e_{n_{j}}-e_{m_{j}}\right)=0 .
$$

Es bien sabido que el rango de una matriz de incidencia $\mathcal{Q}$ con $\left|V_{\mathcal{L}}\right|$ vértices es igual a $\left|V_{\mathcal{L}}\right|-k$, donde $k$ es el número de componentes conexas de la correspondiente gráfica de interacción $\mathcal{G}(\mathcal{L})$. Por lo tanto, se obtiene que $\operatorname{nulidad}(\mathcal{Q})=\left|E_{\mathcal{L}}\right|-$ $\left|V_{\mathcal{L}}\right|+k$. El número máximo de soluciones $\left|E_{\mathcal{L}}\right|-\left|V_{\mathcal{L}}\right|+k$ se alcanza cuando el operador de interacción no se anula en cada vértice de un conjunto completo de ciclos. Esto termina la prueba. 
Los elementos del $\operatorname{ker}(\mathcal{Q})$ serán llamados vectores de corrientes admisibles.

Corolario 2.1. Asuma que para cada vértice de un conjunto completo de ciclos de longitud mínima (con sólo tres vértices), los correspondientes elementos de matriz del operador de interacción no se anulan. Entonces, existe una base $\left\{C_{i j}\right\}_{1 \leq j<i \leq d-1}$ del $\operatorname{ker}(\mathcal{Q})$ asociada con ese conjunto de ciclos. Consecuentemente, cada solución $\sqrt{d e}$ (2.2) puede ser escrita en la forma

$$
\rrbracket=\sum_{1 \leq j<i \leq d-1} J_{i j} C_{i j}
$$

con $J_{i j} \in \mathbb{R}$ para cada $i, j \in\{1,2, . ., d-1\} e i>j$.

Demostración. Bajo las hipótesis, para cada $i, j \in\{1,2, \ldots, d-1\}$ con $i>j$ se definen las componentes $c_{\epsilon_{k} \epsilon_{l}}^{(i, j)}$ del vector $C_{i, j}=\left(c_{\epsilon_{k}, \epsilon_{l}}^{(i, j)}\right)_{0 \leq k, l \leq d-1}$ como sigue:

$$
c_{\epsilon_{k}, \epsilon_{l}}^{(i, j)}=\left\{\begin{array}{cl}
1 & \text { si }\left(\epsilon_{k}, \epsilon_{l}\right)=\left(\epsilon_{i}, \epsilon_{j}\right) \\
1 & \text { si }\left(\epsilon_{k}, \epsilon_{l}\right)=\left(\epsilon_{j}, \epsilon_{0}\right) \\
-1 & \text { si }\left(\epsilon_{k}, \epsilon_{l}\right)=\left(\epsilon_{i}, \epsilon_{0}\right) \\
0 & \text { en otro caso }
\end{array}\right.
$$

Cada $C_{i, j}$ es un vector asociado al ciclo $c=\left(\epsilon_{0} \epsilon_{i} \epsilon_{j}\right)$. Por lo tanto, por $(i)$ del teorema anterior, $C_{i, j}$ es una solución de 2 2.2. . Asimismo, $\operatorname{nulidad}(\mathcal{Q})=\frac{(d-1)(d-2)}{2}$ (donde $d=\operatorname{dim}(\mathrm{h})$ ) y el conjunto $\mathcal{C}=\left\{C_{i, j}: 1 \leq j<i \leq d-1\right\}$ es linealmente independiente, pues para cada $(k, l), \quad 1 \leq k, l \leq d-1$ se tiene que

$$
0=\left(\sum_{1 \leq j<i \leq d-1} a_{i, j} C_{i, j}\right)_{\epsilon_{k}, \epsilon_{l}}=\sum_{1 \leq j<i \leq d-1} a_{i, j} c_{\epsilon_{k}, \epsilon_{l}}^{(i, j)}=a_{k, l}
$$

Además, $|\mathcal{C}|=\frac{(d-1)(d-2)}{2}$. Esto finaliza la prueba.

Bajo las hipótesis del corolario 2.1, fijando el orden lexicográfico en $\mathcal{C}$ y después de reenumerar, podemos escribir cada vector de corriente $\mathbb{l}$ en la forma

$$
\mathbb{J}=\sum_{1 \leq k \leq \mathrm{d}} t_{k} C_{k}
$$


con $\mathrm{d}:=|\mathcal{C}|$. Identificando cada vector $C_{k}=C_{i, j}$ con el correspondiente ciclo y nombrando al número real $t_{k}$ la corriente (constante) o flujo a lo largo del ciclo $C_{k}$. Por lo tanto, en el caso cuando todas las componentes del operador de interacción coinciden con una constante diferente de cero, el resultado $(i)$ del teorema2.1 puede ser reescrito como: Los generadores de Markov del tipo límite de acoplamiento débil tienen estados estacionarios con corrientes constantes en ciclos básicos.

Suponga que un vector de corriente $\sqrt{ }$ es asociado con un estado estacionario de $\mathcal{L}_{*}$, así que las coordenadas de $\sqrt{ }$ y los valores propios de $\rho$ satisfacen el sistema lineal $\Gamma \rho=\sqrt[J]{ }$. Por lo tanto, bajo las hipótesis del corolario 2.1. si

$$
\mathbb{J}=\left(J_{\epsilon_{m_{j}} \epsilon_{n_{j}}}^{\left(\omega_{i}\right)}\right)_{1 \leq i \leq d, 1 \leq j \leq l_{i}}
$$

entonces, para cada $1 \leq k \leq \mathrm{d}$ se tiene que $J_{\epsilon_{m_{j}} \epsilon_{n_{j}}}^{\left(\omega_{i}\right)}=t_{k}$ si $C_{k}$ es el vector asociado con el ciclo $\left(\epsilon_{0} \epsilon_{n_{j}} \epsilon_{m_{j}}\right)$ y, $J_{\epsilon_{m_{j}} \epsilon_{n_{j}}}^{\left(\omega_{i}\right)}$ es una combinación lineal de los vectores en $\mathcal{C}$ en otro caso. De hecho, si $J_{\epsilon_{m_{j}} \epsilon_{n_{j}}}^{\left(\omega_{i}\right)}$ no corresponde con un ciclo básico, entonces

$$
J_{\epsilon_{m_{j}} \epsilon_{n_{j}}}^{\left(\omega_{i}\right)}=\left(\sum_{1 \leq k \leq \mathrm{d}} t_{k} C_{k}\right)_{\epsilon_{m_{j} \epsilon_{n_{j}}}}=\sum_{1 \leq k \leq \mathrm{d}} \zeta_{k}^{(i, j)} t_{k}
$$

donde $\zeta_{k}^{(i, j)} \in\{1,-1\}$

Nótese que el sistema $\Gamma \rho=\rrbracket$ es un sistema de ecuaciones lineales de dimensión $\frac{d(d-1)}{2} \times d$, que está sobredeterminado cuando $d>3$. Sin embargo, bajo condiciones naturales sobre el operador de interacción $D$ y un vector de corriente admisible $\mathbb{J}$, se demuestra la existencia de estados invariantes para cada $d \geq 3$.

Teorema 2.2. Sean $d \geq 3$ y $H \in \mathcal{B}\left(\mathbb{C}^{d}\right)$ un Hamiltoniano de referencia no degenerado, entonces:

(i) Si las hipótesis del corolario 2.1 se verifican y $d=3$, existe un estado invariante $\rho$ para cualquier vector de corriente admisible $\sqrt{ }$ si $\operatorname{det}(\Gamma) \neq 0$, donde $\Gamma$ es la matriz del sistema $\Gamma \rho=\sqrt{ }$.

(ii) Si $d>3$, podemos elegir un operador de interacción $D \in \mathcal{B}\left(\mathbb{C}^{d}\right)$ (por lo tanto, un generador de Markov de $W C L T \mathcal{L}_{D}$ ) y un vector de corriente admisible $\mathbb{J}$, de tal forma que exista un estado $\mathcal{L}_{D}$-invariante $\rho$, que no está en equilibrio. 
Demostración. En el caso $d=3$, bajo las hipótesis, el sistema $\Gamma \rho=\rrbracket$ es un sistema cuadrado que tiene una única solución para cualquier vector de corriente admisible $\sqrt{ }$, si la condición (violación de la condición de reversibilidad de Kolmogorov) $\operatorname{det}(\Gamma) \neq 0$ es válida. Esto prueba $(i)$.

Si $d>3$, el sistema $\Gamma \rho=\sqrt{ }$ está sobredeterminado. Tómese cualquier ciclo mínimo, digamos $c=\left(\epsilon_{0} \epsilon_{1} \epsilon_{2}\right)$, elija un operador de interacción $D$ tal que $\left\langle e_{0}, D e_{l}\right\rangle=1, \quad 1 \leq l \leq d-1,\left\langle e_{1}, D e_{2}\right\rangle=1, \mathrm{y}\left\langle e_{i}, D e_{j}\right\rangle=0$ de otra manera. Sea $\rrbracket_{c}$ el correspondiente vector de corriente solución de (2.2). Por lo tanto, se sigue de (2.4) que existen únicamente tres componentes distintas de cero del correspondiente vector de corriente $\mathbb{J}_{c}$, de hecho, todas las componentes de $\mathbb{J}_{c}$ son cero con excepción de $J_{\epsilon_{1} \epsilon_{2}}, J_{\epsilon_{0} \epsilon_{1}}$, y $J_{\epsilon_{0} \epsilon_{2}}$, las cuales son de la forma $\zeta \tau$ con $\zeta= \pm 1$ y $\tau \in \mathbb{R} \backslash\{0\}$. Por lo tanto, estableciendo el orden lexicográfico, todas las ecuaciones en el sistema $\Gamma \rho=\rrbracket$ son cero con excepción de las primeras $d$ ecuaciones. Queda por demostrar que el sistema cuadrado restante tiene una solución. Claramente, se tiene que

$$
\begin{gathered}
\rho_{j}=\frac{\Gamma_{+, \omega_{j}}}{\Gamma_{-, \omega_{j}}} \rho_{0}, \quad \forall 3 \leq j \leq d-1, \\
\rho_{1}=\frac{\Gamma_{+, \omega_{1}}}{\Gamma_{-, \omega_{1}}} \rho_{0}-\frac{\zeta_{1}}{\Gamma_{-, \omega_{1}}} \tau, \\
\rho_{2}=\frac{\Gamma_{+, \omega_{2}}}{\Gamma_{-, \omega_{2}}} \rho_{0}-\frac{\zeta_{2}}{\Gamma_{-, \omega_{2}}} \tau,
\end{gathered}
$$

$\mathrm{y}$

$$
\rho_{1} \Gamma_{+, \omega_{21}}-\rho_{2} \Gamma_{-, \omega_{21}}=\zeta_{21} \tau
$$

$\operatorname{con} \zeta_{1}, \zeta_{2}, \zeta_{21}= \pm 1$. Por lo tanto,

$$
\rho_{0}=\left(-\Gamma_{+, \omega_{21}} \frac{\Gamma_{+, \omega_{1}}}{\Gamma_{-, \omega_{1}}}+\Gamma_{-, \omega_{21}} \frac{\Gamma_{+, \omega_{2}}}{\Gamma_{-, \omega_{2}}}\right)^{-1}\left(\zeta_{21}+\zeta_{1} \Gamma_{+, \omega_{21}}+\zeta_{2} \Gamma_{-, \omega_{21}}\right) \tau .
$$

La condición de traza permite fijar el valor de la corriente (o flujo) $\tau$ de modo que $\rho$ es un estado. Esto finaliza la prueba.

Observación 2.1. Si $\tilde{\Gamma}$ es la matriz del subsistema (2.5)-(2.7) en la demostración del teorema anterior, la condición $\operatorname{det}(\tilde{\Gamma}) \neq 0$, corresponde con la violación de la condición de reversibilidad de Kolmogorov, consecuentemente, el estado invariante no está en equilibrio. Además, de las ecuaciones (2.6)-(2.7) se verifica inmediatamente que el estado invariante no está en equilibrio, ya que $\tau \neq 0$. 


\subsection{Estados estacionarios de generadores de Mar- kov de WCLT en dimensiones bajas}

\subsubsection{Caso $\mathrm{d}=3$}

Sean $d=3, H$ un Hamiltoniano de referencia no degenerado y $\rho$ una función del Hamiltoniano, por la proposición 2.1, $\rho$ es invariante si para cada frecuencia de Bohr $\omega$,

$$
\rho_{\epsilon_{m}} \Gamma_{+, \omega}-\rho_{\epsilon_{n}} \Gamma_{-, \omega}=0, \quad \forall\left(\epsilon_{n}, \epsilon_{m}\right) \in B_{+, \omega} .
$$

Por lo tanto,

$$
\begin{aligned}
& \Gamma_{+, \omega_{1,0}} \rho_{\epsilon_{0}}-\Gamma_{-, \omega_{1,0}} \rho_{\epsilon_{1}}=0 \\
& \Gamma_{+, \omega_{2,0}} \rho_{\epsilon_{0}}-\Gamma_{-, \omega_{2,0}} \rho_{\epsilon_{2}}=0 \\
& \Gamma_{+, \omega_{2,1}} \rho_{\epsilon_{1}}-\Gamma_{-, \omega_{2,1}} \rho_{\epsilon_{2}}=0
\end{aligned}
$$

con coeficientes $\Gamma_{ \pm, \omega}$ 's descritos en (1.3), para cada $\omega \in B_{+}$. Por lo tanto, se tiene que

$$
\begin{aligned}
& \rho_{\epsilon_{1}}=e^{-\beta\left(\omega_{1,0}\right) \omega_{1,0}} \rho_{\epsilon_{0}} \\
& \rho_{\epsilon_{2}}=e^{-\beta\left(\omega_{2,0}\right) \omega_{2,0}} \rho_{\epsilon_{0}}
\end{aligned}
$$

es una solución no trivial de (2.8) si la condición de reversibilidad de Kolmogorov $e^{-\beta\left(\omega_{2,0}\right) \omega_{2,0}}=e^{-\beta\left(\omega_{2,1}\right) \omega_{2,1}} e^{-\beta\left(\omega_{1,0}\right) \omega_{1,0}}$ se satisface. Consecuentemente,

$$
\rho=\rho_{\epsilon_{0}}\left(\left|e_{0}\right\rangle\left\langle e_{0}\left|+e^{-\beta\left(\omega_{1,0}\right) \omega_{1,0}}\right| e_{1}\right\rangle\left\langle e_{1}\left|+e^{-\beta\left(\omega_{2,0}\right) \omega_{2,0}}\right| e_{2}\right\rangle\left\langle e_{2}\right|\right) .
$$

La condición $1=\operatorname{tr}(\rho)=\rho_{\epsilon_{0}}+\rho_{\epsilon_{1}}+\rho_{\epsilon_{2}}$, permite fijar el valor de $\rho_{\epsilon_{0}}$, de hecho, esta condición implica que

$$
\rho_{\epsilon_{0}}=\left(1+e^{-\beta\left(\omega_{1,0}\right) \omega_{1,0}}+e^{-\beta\left(\omega_{2,0}\right) \omega_{2,0}}\right)^{-1} .
$$

Reescribiendo el Hamiltoniano en términos de las frecuencias, se obtiene que

$$
{ }^{\natural} H=0\left|e_{0}\right\rangle\left\langle e_{0}\left|+\omega_{1,0}\right| e_{1}\right\rangle\left\langle e_{1}\left|+\omega_{2,0}\right| e_{2}\right\rangle\left\langle e_{2}\right| .
$$

Entonces,

$$
e^{-\beta(H) H}=\left|e_{0}\right\rangle\left\langle e_{0}\left|+e^{-\beta\left(\omega_{1,0}\right) \omega_{1,0}}\right| e_{1}\right\rangle\left\langle e_{1}\left|+e^{-\beta\left(\omega_{2,0}\right) \omega_{2,0}}\right| e_{2}\right\rangle\left\langle e_{2}||^{5}\right.
$$

${ }^{4}$ La ecuación 2.9 es una traslación del Hamiltoniano de referencia por $-\epsilon_{0} \mathbb{1}$, es decir, $H-\epsilon_{0} \mathbb{1}$.

${ }^{5} \mathrm{La}$ función $\beta(H) H=\beta(0) 0\left|e_{0}\right\rangle\left\langle e_{0}\left|+\beta\left(\omega_{1,0}\right) \omega_{1,0}\right| e_{1}\right\rangle\left\langle e_{1}\left|+\beta\left(\omega_{2,0}\right) \omega_{2,0}\right| e_{2}\right\rangle\left\langle e_{2}\right|$. 
Consecuentemente,

$$
\rho=\frac{1}{Z} e^{-\beta(H) H},
$$

donde

$$
Z=1+e^{-\beta\left(\omega_{1,0}\right) \omega_{1,0}}+e^{-\beta\left(\omega_{2,0}\right) \omega_{2,0}}=\operatorname{tr}\left(e^{-\beta(H) H}\right) .
$$

Por lo tanto, se concluye que $\rho$ es un estado de Gibbs.

\subsubsection{Caso $d=4$}

Sea $d=4$ y $H \in \mathcal{B}\left(\mathbb{C}^{4}\right)$ un Hamiltoniano de referencia no degenerado, es decir,

$$
H=\sum_{\epsilon_{i} \in S p(H)} \epsilon_{i} P_{\epsilon_{i}}=\sum_{i=0}^{3} \epsilon_{i}\left|e_{i}\right\rangle\left\langle e_{i}\right|,
$$

donde $0 \leq \epsilon_{0}<\epsilon_{1}<\epsilon_{2}<\epsilon_{3}$ son los cuatro valores propios de $H$ y $\left\{\left|e_{i}\right\rangle\right\}_{0 \leq i \leq 3}$ es la base canónica de $\mathbb{C}^{4}$. En este caso, hay a lo más seis frecuencias de Bohr y se asume que todas las frecuencias de Bohr son diferentes, así que

$$
B_{+}=\left\{\omega_{1,0}, \omega_{2,0}, \omega_{3,0}, \omega_{2,1}, \omega_{3,1}, \omega_{3,2}\right\},
$$

donde $\omega_{i j}=\epsilon_{i}-\epsilon_{j}, \quad 0 \leq j<i \leq d-1$. Sea $\mathcal{L}$ un generador de Markov de WCLT asociado con $H$ y $\rho$ una función del Hamiltoniano. $\mathcal{L}_{*}(\rho)=0$ es equivalente al sistema $\mathcal{Q} \mathbb{J}=0$, donde $\mathcal{Q}$ es la matriz de incidencia de la correspondiente gráfica de interacción $\mathcal{G}(\mathcal{L})$ y $\mathbb{J}=\left(J_{\epsilon_{m_{j}} \epsilon_{n_{j}}}^{\left(\omega_{i}\right)}\right)_{1 \leq i \leq d, 1 \leq j \leq l_{i}}$ es el vector de corriente que satisface el sistema $\Gamma \rho=\sqrt{ }$, explícitamente,

$$
J_{\epsilon_{m_{j}} \epsilon_{n_{j}}}^{\left(\omega_{i}\right)}=\left(\rho_{\epsilon_{m_{j}}} \Gamma_{+, \omega_{i}}-\rho_{\epsilon_{n_{j}}} \Gamma_{-, \omega_{i}}\right)\left|\left\langle e_{m_{j}}, D e_{n_{j}}\right\rangle\right|^{2}
$$

para $\left(\epsilon_{m_{j}}, \epsilon_{n_{j}}\right) \in B_{+, \omega_{i}}$. Además, suponga que todas las componentes del operador de interacción $D$ coinciden, digamos $\left|\left\langle e_{m_{j}}, D e_{n_{j}}\right\rangle\right|^{2}=1$ para todo $0 \leq j \leq d-1$. Por lo tanto, el conjunto solución de $\mathcal{Q} \rrbracket=0$ es

$$
\left\{\mathbb{l}=r C_{21}+s C_{31}+t C_{32}: r, s, t \in \mathbb{R}\right\},
$$

donde el conjunto

$\mathcal{C}=\left\{C_{21}=(1,-1,0,1,0,0), C_{31}=(1,0,-1,0,1,0), C_{32}=(0,1,-1,0,0,1)\right\}$

es la base $\operatorname{del} \operatorname{ker}(\mathcal{Q})$. Equivalentemente, cada solución $\rrbracket$ de $\mathcal{Q} \rrbracket=0$ es de la forma 


$$
\mathbb{J}=(r+s, t-r,-s-t, r, s, t)
$$

con $r, s, t \in \mathbb{R}$. Además, los ciclos básicos asociados a la base $\mathcal{C}=\left\{C_{21}, C_{31}, C_{32}\right\}$ son: $c_{21}=\left(\epsilon_{0} \epsilon_{2} \epsilon_{1}\right), c_{31}=\left(\epsilon_{0} \epsilon_{3} \epsilon_{1}\right)$ y $c_{32}=\left(\epsilon_{0} \epsilon_{3} \epsilon_{2}\right)$. Posteriormente, fijando el orden lexicográfico en el sistema (2.10), para cada vector de corriente admisible $\sqrt{ }$ asociado con $\rho$ y la matriz $\Gamma$ satisfaciendo la ecuación (2.10), el sistema no homogéneo $\Gamma \rho=\sqrt{ }$ está sobredeterminado porque tiene seis ecuaciones y cuatro incógnitas, explícitamente,

$$
\left(\begin{array}{cccc}
-\Gamma_{+, \omega_{1,0}} & \Gamma_{-, \omega_{1,0}} & 0 & 0 \\
-\Gamma_{+, \omega_{2,0}} & 0 & \Gamma_{-, \omega_{2,0}} & 0 \\
-\Gamma_{+, \omega_{3,0}} & 0 & 0 & \Gamma_{-, \omega_{3,0}} \\
0 & -\Gamma_{+, \omega_{2,1}} & \Gamma_{-, \omega_{2,1}} & 0 \\
0 & -\Gamma_{+, \omega_{3,1}} & 0 & \Gamma_{-, \omega_{3,1}} \\
0 & 0 & -\Gamma_{+, \omega_{3,2}} & \Gamma_{-, \omega_{3,2}}
\end{array}\right)\left(\begin{array}{c}
\rho_{0} \\
\rho_{1} \\
\rho_{2} \\
\rho_{3}
\end{array}\right)=\left(\begin{array}{c}
J_{\epsilon_{1} \epsilon_{0}} \\
J_{\epsilon_{2} \epsilon_{0}} \\
J_{\epsilon_{3} \epsilon_{0}} \\
J_{\epsilon_{2} \epsilon_{1}} \\
J_{\epsilon_{3} \epsilon_{1}} \\
J_{\epsilon_{3} \epsilon_{2}}
\end{array}\right)
$$

Considere la matriz $\bar{\Gamma}=[\Gamma \mid \mathbb{J}]$. Después de aplicar eliminación Gaussiana a la matriz $\bar{\Gamma}$ se obtiene

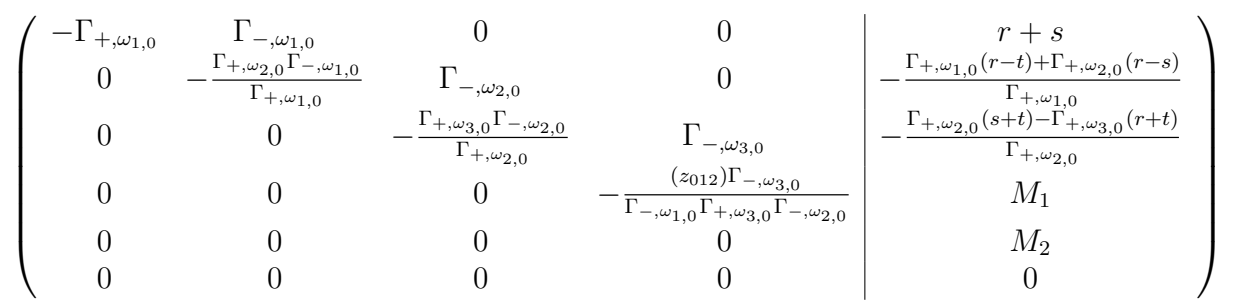

donde

$$
\begin{aligned}
z_{012} & :=\Gamma_{+, \omega_{1,0}} \Gamma_{-, \omega_{2,0}} \Gamma_{+, \omega_{2,1}}-\Gamma_{-, \omega_{1,0}} \Gamma_{+, \omega_{2,0}} \Gamma_{-, \omega_{2,1}} \\
M_{1} & :=\frac{\sigma_{1} r+\sigma_{2} s+\sigma_{3} t}{\Gamma_{-, \omega_{1,0}} \Gamma_{+, \omega_{3,0}} \Gamma_{-, \omega_{2,0}}} \\
M_{2} & :=\frac{\theta_{1} r+\theta_{2} s+\theta_{3} t}{\left(z_{012}\right) \Gamma_{-, \omega_{3,0}}}
\end{aligned}
$$

$\mathrm{y}$

$$
\begin{aligned}
\sigma_{1} & =\Gamma_{-, \omega_{1,0}} \Gamma_{+, \omega_{3,0}} \Gamma_{-, \omega_{2,0}}+\Gamma_{-, \omega_{1,0}} \Gamma_{+, \omega_{3,0}} \Gamma_{-, \omega_{2,1}}-\Gamma_{-, \omega_{2,0}} \Gamma_{+, \omega_{3,0}} \Gamma_{+, \omega_{2,1}} \\
\sigma_{2} & =z_{012}+\Gamma_{-, \omega_{2,0}} \Gamma_{+, \omega_{3,0}} \Gamma_{+, \omega_{2,1}} \\
\sigma_{3} & =z_{012}-\Gamma_{-, \omega_{1,0}} \Gamma_{+, \omega_{3,0}} \Gamma_{-, \omega_{2,1}}
\end{aligned}
$$




$$
\begin{gathered}
\theta_{1}=z_{031}\left(\Gamma_{-, \omega_{2,0}}+\Gamma_{-, \omega_{2,1}}\right)+z_{0312} \\
\theta_{2}=z_{012}\left(\Gamma_{-, \omega_{3,0}}+\Gamma_{-, \omega_{3,1}}\right)+z_{0312} \\
\theta_{3}=z_{012} \Gamma_{-, \omega_{3,1}}-z_{031} \Gamma_{-, \omega_{2,1}} \\
z_{0312}=\Gamma_{-, \omega_{2,0}} \Gamma_{+, \omega_{3,0}} \Gamma_{+, \omega_{2,1}} \Gamma_{-, \omega_{3,1}}-\Gamma_{+, \omega_{2,0}} \Gamma_{-, \omega_{3,0}} \Gamma_{-, \omega_{2,1}} \Gamma_{+, \omega_{3,1}} \\
z_{031}=\Gamma_{-, \omega_{1,0}} \Gamma_{+, \omega_{3,0}} \Gamma_{-, \omega_{3,1}}-\Gamma_{+, \omega_{1,0}} \Gamma_{-, \omega_{3,0}} \Gamma_{+, \omega_{3,1}}
\end{gathered}
$$

Por lo tanto, el sistema de ecuaciones lineales no homogéneo $\Gamma \rho=\sqrt{ }$ está determinado (y es un sistema cuadrado) si y sólo si se satisfacen las condiciones siguientes:

$$
\begin{array}{r}
z_{012}:=\Gamma_{+, \omega_{1,0}} \Gamma_{-, \omega_{2,0}} \Gamma_{+, \omega_{2,1}}-\Gamma_{-, \omega_{1,0}} \Gamma_{+, \omega_{2,0}} \Gamma_{-, \omega_{2,1}} \neq 0 \\
\Gamma_{-, \omega_{3,0}} \neq 0 \\
\theta_{1} r+\theta_{2} s+\theta_{3} t=0
\end{array}
$$

Bajo las condiciones 2.11, el sistema restante es un sistema cuadrado $\widetilde{\Gamma} \rho=\sqrt{ }$ que tiene una única solución si y sólo si $\operatorname{det}(\widetilde{\Gamma}) \neq 0$. Mediante cálculos directos se obtiene que

$$
\rho_{i}=E_{i}+F_{i} r
$$

para todo $0 \leq i \leq 3$, donde $E_{i}, F_{i}, 0 \leq i \leq 3$ son funciones de las $\theta$ 's y de los coeficientes $\Gamma_{ \pm, \omega_{k, l}}$ 's. La positividad de los valores propios de $\rho$ implica que

$$
r>\frac{-E_{i}}{F_{i}}=: K_{i}
$$

para todo $i=0,1,2,3$. La condición $1=\operatorname{tr}(\rho)$ fija el valor de $r=(1-$ $\left.\sum_{j=0}^{3} E_{j}\right)\left(\sum_{j=0}^{3} F_{j}\right)^{-1}$. El parámetro $r$ corresponde al valor de la microcorriente (o flujo).

La condición $\operatorname{det}(\widetilde{\Gamma})=z_{012} \neq 0$ corresponde con la violación de la condición de reversibilidad de Kolmogorov. Por lo tanto, el estado no satisface una condición de balance detallado, pero si de equilibrio local. A este tipo de estados se les llamará (de ahora en adelante), estados localmente de Gibbs.

\subsection{Existencia de estados estacionarios de balance detallado local}

Existen generadores de Markov del tipo límite de acoplamiento débil con estados estacionarios que no satisfacen la condición de balance detallado, pero son 
de equilibrio local. En esta sección, se muestra como construir tales generadores eligiendo operadores de interacción $D$ apropiados y coeficientes $\Gamma_{ \pm, \omega}$ 's.

Considere estados que satisfagan la condición $\rho D_{\omega}=c_{\omega} D_{\omega} \rho$ con $c_{\omega}=\frac{\Gamma_{-, \omega}}{\Gamma_{+, \omega}}$, para casi todo $\omega$ con excepción de algunos $\omega$ 's. Se dice que los estados de esta clase satisfacen una condición de balance detallado local o que son estados de balance detallado local. De hecho, la terminología anterior está motivada por elegir $c_{\omega}=$ $\frac{\Gamma_{-, \omega}}{\Gamma_{+, \omega}}$ para casi todo $\omega$ con la excepción de las frecuencias asociadas a un ciclo $\left(\epsilon_{0} \epsilon_{j} \epsilon_{i}\right), j<i$. Más precisamente, el resultado se prueba en el teorema siguiente.

Teorema 2.3. Sea $\mathcal{L}$ un generador de Markov de WCLT con Hamiltoniano de referencia $H=\sum_{n} \epsilon_{n} P_{n}$ y denote por $r_{n}=r\left(\epsilon_{n}\right)<\infty$ el rango de $P_{n}$. Elija el ciclo $\left(\epsilon_{0} \epsilon_{1} \epsilon_{2}\right)$, sea $D=|\chi\rangle\langle\chi|$ con $\chi$ el vector de entrelazamiento máximo (maximally entangled) $\chi=\sum_{l} e_{l}$, donde $\left\{e_{0}, \cdots, e_{n}, \cdots\right\}$ es la base ortonormal del Hamiltoniano $H$. Entonces, existe $\mathcal{L}_{*}$ un generador de Markov de WCLT con un estado estacionario de balance detallado local

$$
\rho=\sum_{n \geq 0} \rho_{n} P_{n} \in\{H\}^{\prime \prime}
$$

satisfaciendo $\rho D_{\omega}=c_{\omega} D_{\omega} \rho$, con $c_{\omega}=\frac{\Gamma_{-, \omega}}{\Gamma_{+, \omega}}$ para casi todo $\omega$ con la excepción de $\omega_{10}=\epsilon_{1}-\epsilon_{0}, \omega_{20}=\epsilon_{2}-\epsilon_{0} y \omega_{21}=\epsilon_{2}-\epsilon_{1}, \quad \epsilon_{0}<\epsilon_{1}<\epsilon_{2}$.

Demostración. Asuma que la condición $\rho D_{\omega}=c_{\omega} D_{\omega} \rho \operatorname{con} c_{\omega}=\frac{\Gamma_{-, \omega}}{\Gamma_{+, \omega}}$, es válida para todas las frecuencias $\omega \neq \omega_{i j}$, con $i>j, i, j=0,1,2$. Se verifica inmediatamente que

$$
\mathcal{L}_{\omega *}(\rho)=0, \quad \forall \omega \neq \omega_{i j}, \quad i>j, i, j=0,1,2 .
$$

Además, cálculos simples muestran que la condición $\rho D_{\omega}=c_{\omega} D_{\omega} \rho$ implica que $\rho_{n}=\frac{\Gamma_{+, \omega_{n 0}}}{\Gamma_{-, \omega_{n 0}}} \rho_{0}$ para $n \geq 3$, consecuentemente, todos estos valores propios de $\rho$ se pueden calcular en términos de $\rho_{0}$.

Por lo tanto, resta calcular los primeros tres valores propios de $\rho$ tal que

$$
\mathcal{L}_{*}(\rho)=\mathcal{L}_{\omega_{10 *}}(\rho)+\mathcal{L}_{\omega_{20} *}(\rho)+\mathcal{L}_{\omega_{21} *}(\rho)=0
$$

con

$$
\begin{aligned}
\mathcal{L}_{\omega *}(\rho)= & \left(-\frac{\Gamma_{-, \omega}}{2}\left\{D_{\omega}^{*} D_{\omega}, \rho\right\}+\Gamma_{+, \omega} D_{\omega}^{*} \rho D_{\omega}\right) \\
& +\left(-\frac{\Gamma_{+, \omega}}{2}\left\{D_{\omega} D_{\omega}^{*}, \rho\right\}+\Gamma_{-, \omega} D_{\omega} \rho D_{\omega}^{*}\right)-i\left[\Delta_{\omega}, \rho\right]
\end{aligned}
$$


para $\omega=\omega_{i j}, \quad i>j, i, j=0,1,2$.

La condición $\rho D_{\omega}=c_{\omega} D_{\omega} \rho$ implica que $\left[\rho, D_{\omega} D_{\omega}^{*}\right]=0=\left[\rho, D_{\omega}^{*} D_{\omega}\right]$, consecuentemente $\left[\Delta_{\omega}, \rho\right]=0 \mathrm{y}$

$$
\begin{aligned}
\mathcal{L}_{\omega *}(\rho)= & -\Gamma_{-, \omega} D_{\omega}^{*} D_{\omega} \rho+c_{\omega} \Gamma_{+, \omega} D_{\omega}^{*} D_{\omega} \rho \\
& -\Gamma_{+, \omega} D_{\omega} D_{\omega}^{*} \rho+c_{\omega}^{-1} \Gamma_{-, \omega} D_{\omega} D_{\omega}^{*} \rho .
\end{aligned}
$$

Ahora, para cada $\omega=\omega_{i j}=\epsilon_{i}-\epsilon_{j}, \quad i>j, i, j=0,1,2$ se tiene que

$$
\begin{aligned}
& D_{\omega}^{*} D_{\omega}=P_{i} D^{*} P_{j} D P_{i}=P_{i}|\chi\rangle\left\langle\chi\left|P_{j}\right| \chi\right\rangle\left\langle\chi\left|P_{i}=\left\|P_{j} \chi\right\|^{2}\right| P_{i} \chi\right\rangle\left\langle P_{i} \chi\right| \\
& D_{\omega} D_{\omega}^{*}=P_{j} D P_{i} D^{*} P_{j}=P_{j}|\chi\rangle\left\langle\chi\left|P_{i}\right| \chi\right\rangle\left\langle\chi\left|P_{j}=\left\|P_{i} \chi\right\|^{2}\right| P_{j} \chi\right\rangle\left\langle P_{j} \chi\right| .
\end{aligned}
$$

Por lo tanto, mediante cálculos directos y utilizando que $\rho P_{n} \chi=\rho_{n} P_{n} \chi$, se obtiene que $\mathcal{L}_{*}(\rho)=0$ si y sólo si

$$
\begin{aligned}
0= & \left(-\Gamma_{-, \omega_{10}}+c_{\omega_{10}} \Gamma_{+, \omega_{10}}\right)\left\|P_{1} \chi\right\|^{2} \rho_{0}\left|P_{0} \chi\right\rangle\left\langle P_{0} \chi\right| \\
& +\left(-\Gamma_{+, \omega_{10}}+c_{\omega_{10}}^{-1} \Gamma_{-, \omega_{10}}\right)\left\|P_{0} \chi\right\|^{2} \rho_{1}\left|P_{1} \chi\right\rangle\left\langle P_{1} \chi\right| \\
& +\left(-\Gamma_{-, \omega_{20}}+c_{\omega_{20}} \Gamma_{+, \omega_{20}}\right)\left\|P_{2} \chi\right\|^{2} \rho_{0}\left|P_{0} \chi\right\rangle\left\langle P_{0} \chi\right| \\
& +\left(-\Gamma_{+, \omega_{20}}+c_{\omega_{20}}^{-1} \Gamma_{-, \omega_{20}}\right)\left\|P_{0} \chi\right\|^{2} \rho_{2}\left|P_{2} \chi\right\rangle\left\langle P_{2} \chi\right| \\
& +\left(-\Gamma_{-, \omega_{21}}+c_{\omega_{21}} \Gamma_{+, \omega_{21}}\right)\left\|P_{2} \chi\right\|^{2} \rho_{1}\left|P_{1} \chi\right\rangle\left\langle P_{1} \chi\right| \\
& +\left(-\Gamma_{+, \omega_{21}}+c_{\omega_{21}}^{-1} \Gamma_{-, \omega_{21}}\right)\left\|P_{1} \chi\right\|^{2} \rho_{2}\left|P_{2} \chi\right\rangle\left\langle P_{2} \chi\right|
\end{aligned}
$$

Nótese que $\left\|P_{n} \chi\right\|=r\left(\epsilon_{n}\right)=r_{n}$ es el rango de $P_{n}$. Entonces, agrupando términos y de la ortogonalidad de los rangos, se verifica que la ecuación anterior es equivalente al sistema

$$
\begin{aligned}
& r_{1}^{2} c_{\omega_{10}} \Gamma_{+, \omega_{10}}+r_{2}^{2} c_{\omega_{20}} \Gamma_{+, \omega_{20}}=r_{1}^{2} \Gamma_{-, \omega_{10}}+r_{2}^{2} \Gamma_{-, \omega_{20}} \\
& r_{0}^{2} c_{\omega_{10}}^{-1} \Gamma_{-, \omega_{10}}+r_{2}^{2} c_{\omega_{21}} \Gamma_{+, \omega_{21}}=r_{0}^{2} \Gamma_{+, \omega_{10}}+r_{2}^{2} \Gamma_{-, \omega_{21}} \\
& r_{0}^{2} c_{\omega_{20}}^{-1} \Gamma_{-, \omega_{20}}+r_{1}^{2} c_{\omega_{21}}^{-1} \Gamma_{-, \omega_{21}}=r_{0}^{2} \Gamma_{+, \omega_{20}}+r_{1}^{2} \Gamma_{+, \omega_{21}}
\end{aligned}
$$

Además,

$$
\begin{aligned}
& \rho_{0} D_{\omega_{10}}=\rho D_{\omega_{10}}=c_{\omega_{10}} D_{\omega_{10}} \rho=\rho_{1} c_{\omega_{10}} D_{\omega_{10}} \Longrightarrow \rho_{1}=c_{\omega_{10}}^{-1} \rho_{0} \\
& \rho_{0} D_{\omega_{20}}=\rho D_{\omega_{20}}=c_{\omega_{20}} D_{\omega_{20}} \rho=\rho_{2} c_{\omega_{20}} D_{\omega_{20}} \Longrightarrow \rho_{2}=c_{\omega_{20}}^{-1} \rho_{0} \\
& \rho_{1} D_{\omega_{21}}=\rho D_{\omega_{21}}=c_{\omega_{21}} D_{\omega_{21}} \rho=\rho_{2} c_{\omega_{21}} D_{\omega_{21}} \Longrightarrow \rho_{1}=c_{\omega_{21}} \rho_{2}
\end{aligned}
$$


Consecuentemente,

$$
c_{\omega_{21}}=\frac{\rho_{1}}{\rho_{2}}=\frac{c_{\omega_{20}}}{c_{\omega_{10}}}
$$

De la primera ecuación en el sistema (2.13) se obtiene que

$$
c_{\omega_{20}}=\left(\left(r_{1} / r_{2}\right)^{2}\left(\Gamma_{-, \omega_{10}}-c_{\omega_{10}} \Gamma_{+, \omega_{10}}\right)+\Gamma_{-, \omega_{20}}\right) \Gamma_{+, \omega_{20}}^{-1}
$$

y de la tercera ecuación, usando la relación (2.14, se obtiene que

$$
c_{\omega_{20}}=\left(r_{0}^{2} \Gamma_{-, \omega_{20}}+r_{1}^{2} c_{\omega_{10}} \Gamma_{-, \omega_{21}}\right)\left(r_{0}^{2} \Gamma_{+, \omega_{20}}+r_{1}^{2} \Gamma_{+, \omega_{21}}\right)^{-1} .
$$

Las últimas dos ecuaciones implican que

$$
c_{\omega_{10}}=\frac{r_{0}^{2} \Gamma_{-, \omega_{10}} \Gamma_{+, \omega_{20}}+\left(r_{1}^{2} \Gamma_{-, \omega_{10}}+r_{2}^{2} \Gamma_{-, \omega_{20}}\right) \Gamma_{+, \omega_{21}}}{\left(r_{0}^{2} \Gamma_{+, \omega_{20}}+r_{1}^{2} \Gamma_{+, \omega_{21}}\right) \Gamma_{+, \omega_{10}}+r_{2}^{2} \Gamma_{-, \omega_{21}} \Gamma_{+, \omega_{20}}} .
$$

Por lo tanto, de este valor de $c_{\omega_{10}}$ y de la primera ecuación en 2.13, se obtiene que

$$
\begin{aligned}
c_{\omega_{20}} & =\left(r_{1}^{2} \Gamma_{-, \omega_{10}}+r_{2}^{2} \Gamma_{-, \omega_{20}}\right)\left(r_{2}^{2} \Gamma_{+, \omega_{20}}\right)^{-1} \\
& -\left(r_{1}^{2}\left(\frac{r_{0}^{2} \Gamma_{-, \omega_{10}} \Gamma_{+, \omega_{20}}+\left(r_{1}^{2} \Gamma_{-, \omega_{10}}+r_{2}^{2} \Gamma_{-, \omega_{20}}\right) \Gamma_{+, \omega_{21}}}{\left(r_{0}^{2} \Gamma_{+, \omega_{20}}+r_{1}^{2} \Gamma_{+, \omega_{21}}\right) \Gamma_{+, \omega_{10}}+r_{2}^{2} \Gamma_{-, \omega_{21}} \Gamma_{+, \omega_{20}}}\right) \Gamma_{+, \omega_{10}}\right)\left(r_{2}^{2} \Gamma_{+, \omega_{20}}\right)^{-1} .
\end{aligned}
$$

El valor restante $c_{\omega_{21}}$ se puede calcular a partir de (2.14). La segunda ecuación en (2.13) produce una condición de compatibilidad que deben satisfacer los coeficientes $\Gamma_{ \pm, \omega_{i j}}$ 's. Esta condición de compatibilidad distingue aquellos generadores de Markov de WCLT que tienen estados de balance detallado local de la forma (2.12).

Por lo tanto, si se cumplen las condiciones anteriores, se obtiene un estado invariante $\rho$ de la forma (2.12), donde los valores propios $\rho_{n}$ para $n \geq 3$ están dados por las relaciones de balance detallado $\rho_{n}=\frac{\Gamma_{+, \omega_{n 0}}}{\Gamma_{-, \omega_{n 0}}} \rho_{0}$ y los valores propios restantes satisfacen $\rho_{1}=c_{\omega_{10}}^{-1} \rho_{0}$ y $\rho_{2}=c_{\omega_{20}}^{-1} \rho_{0}$, así que

$$
\begin{aligned}
\rho & =\rho_{0}\left(P_{0}+c_{\omega_{10}}^{-1} P_{1}+c_{\omega_{20}}^{-1} P_{2}+\sum_{n \geq 3} \frac{\Gamma_{+, \omega_{n 0}}}{\Gamma_{-, \omega_{n 0}}} P_{n}\right) \\
& =\rho_{0}\left(P_{0}+c_{\omega_{10}}^{-1} P_{1}+c_{\omega_{20}}^{-1} P_{2}+\sum_{n \geq 3} e^{-\beta\left(\omega_{n 0}\right) \omega_{n o}} P_{n}\right) .
\end{aligned}
$$


El primer valor propio $\rho_{0}$ se determina a partir de la condición de normalización $\operatorname{tr}(\rho)=1$. En efecto,

$$
\rho_{0}=\left(r_{0}+r_{1} c_{\omega_{10}}^{-1}+r_{2} c_{\omega_{20}}^{-1}+\sum_{n \geq 3} r_{n} e^{-\beta\left(\omega_{n 0}\right) \omega_{n o}}\right)^{-1} .
$$

Claramente el operador $\rho$ es positivo. Esto termina la prueba.

El teorema anterior es una versión del teorema 3.1 en [16] para la clase de todos los generadores de Markov de WCLT genéricos. Para aquellos estados estacionarios que no satisfacen la condición de balance detallado (pero de equilibrio local), la pregunta sobre los valores asociados a los flujos (o corrientes) de energía surge naturalmente. Por definición, el flujo de energía (o corriente) entre los niveles de energía $\epsilon_{m}$ y $\epsilon_{n}$ será dado por

$$
J_{m n}=\rho_{m} \Gamma_{+, \omega}-\rho_{n} \Gamma_{-, \omega}
$$

para $\omega=\epsilon_{n}-\epsilon_{m}>0$. Por lo tanto, $J_{m n}=J_{0 n}=0$ para $n \geq 3$ y $m \geq 0$. Pero en los tres primeros niveles de energía, es decir, en el ciclo $c=\left(\epsilon_{0} \epsilon_{1} \epsilon_{2}\right)$ se tienen flujos distintos de cero:

$$
\begin{aligned}
& J_{01}=\rho_{0} \Gamma_{+, \omega_{10}}-\rho_{1} \Gamma_{-, \omega_{10}}=\rho_{0}\left(\Gamma_{+, \omega_{10}}-c_{\omega_{10}}^{-1} \Gamma_{-, \omega_{10}}\right), \\
& J_{02}=\rho_{0} \Gamma_{+, \omega_{20}}-\rho_{2} \Gamma_{-, \omega_{20}}=\rho_{0}\left(\Gamma_{+, \omega_{20}}-c_{\omega_{20}}^{-1} \Gamma_{-, \omega_{20}}\right), \\
& J_{12}=\rho_{1} \Gamma_{+, \omega_{21}}-\rho_{2} \Gamma_{-, \omega_{21}}=\rho_{0}\left(c_{\omega_{10}}^{-1} \Gamma_{+, \omega_{21}}-c_{\omega_{20}}^{-1} \Gamma_{-, \omega_{21}}\right) .
\end{aligned}
$$

En el capítulo siguiente, se presenta nuestra principal contribución sobre la estructura de los estados estacionarios del tipo límite de acoplamiento débil y modelos de transporte cuántico. 


\section{Capítulo 3}

\section{Estados estacionarios de generadores de Markov de WCLT y modelos de transporte cuántico}

En este capítulo se describe la estructura de los estados estacionarios en el aniquilador de todos los operadores de Kraus (u operadores de ruido), de la clase de generadores de Markov del tipo límite de acoplamiento débil introducidos en [1]. En particular, se describe en detalle la estructura de los estados estacionarios de un modelo de transporte cuántico estudiado en [28], en términos de operadores de aniquilación y creación generalizados.

Un espacio natural para buscar estados estacionarios de generadores de Markov de WCLT es el aniquilador de todos los operadores de Kraus u operadores de ruido, definido mediante

$$
\operatorname{Ann}(D):=\left\{\rho: \operatorname{tr}\left(\rho D_{\omega}\right)=0, \text { para cada frecuencia de Bohr } \omega\right\},
$$

que contiene (algunas veces propiamente) al conmutante del Hamiltoniano de referencia $\{H\}^{\prime}$. Se demuestra que los estados estacionarios en $\operatorname{Ann}(D)$ consisten de dos partes, una parte soportada en la intersección de los núcleos de todos los operadores de Kraus y sus adjuntos, denotado por $W_{D}$ y llamado subespacio libre de interacción y, otra parte soportada en $W_{D}^{\perp}$. La parte soportada en $W_{D}$ pertenece a la subálgebra de puntos fijos del semigrupo, la otra parte es mucho más interesante e incluye estados estacionarios de balance detallado, así como también sin balance detallado, pero de balance detallado local. 
Para el modelo de transporte cuántico, los estados estacionarios soportados en $W_{D}^{\perp}$, poseen una estructura muy interesante descrita explícitamente mediante el uso de un operador $Z$, llamado operador de interferencia [28], y su adjunto $Z^{*}$. El operador $Z$ envía el subespacio soporte $P_{2}(\mathrm{~h})$ de la segunda proyección espectral del Hamiltoniano de referencia degenerado $H$, en el subespacio soporte $P_{3}(\mathrm{~h})$ de la tercera proyección espectral. Los operadores $Z$ y $Z^{*}$ realizan transiciones entre $P_{2}(h)$ y $P_{3}(h)$, similar a las transiciones de nacimiento y muerte en los procesos estocásticos clásicos u operadores de creación y aniquilación en el escenario cuántico. Se demuestra en el teorema 3.5 que cualquier estado estacionario soportado en $W_{D}^{\perp} \cap V$, es una combinación convexa de un estado $\sigma$ soportado en un subespacio de $P_{2}(\mathrm{~h})$ y su conjugación por $Z, Z \sigma Z^{*}$, que está soportada en un subespacio de $P_{3}(\mathrm{~h})$. Resulta ser que en el modelo de transporte cuántico de [28], la masa total de probabilidad de cualquier estado inicial puede ser redistribuida cuando $t \rightarrow \infty$, eligiendo valores apropiados de los parámetros del modelo (los coeficientes $\Gamma$ 's), de modo que la mayor parte de la probabilidad total del estado final se concentra en la parte soportada en $P_{3}(\mathrm{~h})$, a pesar de que el soporte del estado inicial no este contenido en $P_{3}(\mathrm{~h})$-hay una "huella" no nula en $P_{2}(\mathrm{~h})$ - (ver observación 3.18 y figura 3.1. Además, la energía del sistema pequeño se incrementa durante la evolución: cuando está inicialmente en un estado soportado en un subespacio distinguido de $P_{2}(\mathrm{~h})$, hay una ganancia de energía cuando $t \rightarrow \infty$ proporcional a $(N-1)=\operatorname{dim} P_{2}(\mathrm{~h})$, véase la observación 3.19 .

La estructura del presente capítulo es la siguiente. En la sección 3.1 se define el aniquilador de todos los operadores de Kraus y se verifica que es un conjunto más grande que $\{H\}^{\prime}$. Posteriormente, en la sección 3.2 se describe a los estados estacionarios con soporte en el subespacio libre de interacción $W_{D}$. En la sección 3.3 se demuestra que la estructura de los estados estacionarios de generadores de Markov del tipo límite de acoplamiento débil, es una combinación convexa de un estado con soporte en el subespacio libre de interacción $W_{D}$ y otro con soporte en el complemento ortogonal $W_{D}^{\perp}$. En la sección 3.4 se muestran algunos ejemplos. Finalmente, en la sección 3.5 se aplican los resultados de la sección 3.3 para estudiar los estados estacionarios del modelo de transporte cuántico, mediante un método considerablemente más corto que el utilizado en [28]. En contraposición con la referencia anterior, donde se asume que cualquier estado estacionario pertenece a $\{H\}^{\prime}$, se comienza con el cálculo de proyecciones subarmónicas y se demuestra que cualquier estado estacionario soportado en $W_{D}^{\perp} \cap V$ pertenece a $\{H\}^{\prime}$, no obstante, existen estados estacionarios en $A n n(D) \backslash\{H\}^{\prime}$ (ver corolario 3.3 y observación 3.15). Además, se identifica el subespacio de recurrencia rápi- 
da, se estudia la propiedad de aproximación al equilibrio para este semigrupo, se caracteriza el dominio de atracción de cualquier estado estacionario, se estudia el transporte de estados y la ganancia de energía.

\subsection{Estados estacionarios en el aniquilador de todos los operadores de Kraus}

En esta sección se estudian los estados estacionarios de generadores de Markov de WCLT que pertenecen al aniquilador de todos los operadores de Kraus, definido como

$$
\operatorname{Ann}(D):=\left\{\rho: \operatorname{tr}\left(\rho D_{\omega}\right)=0, \text { para cada frecuencia de Bohr } \omega\right\} .
$$

Observe que

$$
\{H\}^{\prime \prime} \subseteq\{H\}^{\prime}
$$

con igualdad si $H$ es no degenerado. Además, para la clase de generadores de Markov de WCLT la inclusión

$$
\{H\}^{\prime} \subset \operatorname{Ann}(D)
$$

se verifica. En efecto, sea $\omega \in B_{+}$, para cualquier $\rho \in\{H\}^{\prime}$,

$$
\operatorname{tr}\left(\rho D_{\omega}\right)=\operatorname{tr}\left(\rho \sum_{\left(\varepsilon_{n}, \varepsilon_{m}\right) \in B_{+, \omega}} P_{m} D P_{n}\right)=\sum_{\left(\varepsilon_{n}, \varepsilon_{m}\right) \in B_{+, \omega}} \operatorname{tr}\left(P_{n} P_{m} \rho D\right)=0,
$$

pues $m \neq n$. Sin embargo, existen generadores de Markov de WCLT con estados estacionarios $\rho \in A n n(D) \backslash\{H\}^{\prime}$ como se muestra en el corolario 3.3, observación 3.15 y ejemplo 3.3 al final del capítulo.

Observación 3.1. Nótese que en la definición del Ann (D) 3.1), $\rho \in A n n(D)$ es equivalente a que $\rho \perp D_{\omega}$, para cada frecuencia de Bohr $\omega$, con respecto al producto interno Hilbert-Schmidt.

\subsection{Estados estacionarios con soporte en el subes- pacio libre de interacción}

A continuación, se describe la clase más simple de los estados estacionarios en el aniquilador de todos los operadores de Kraus, dichos estados estacionarios se 
caracterizan por la condición $\rho D_{\omega}=0=D_{\omega} \rho$, para todo $\omega$. El subespacio soporte de estos estados estacionarios está contenido en la intersección de los núcleos de todos los operadores de Kraus; además, estos estados estacionarios no dependen de los coeficientes $\Gamma_{ \pm, \omega}$ 's.

Definición 3.1. (i) Cualquier subconjunto $\Theta \subseteq \mathcal{B}(\mathrm{h})$ es singular si y sólo si

$$
\operatorname{ker} \Theta:=\bigcap_{\theta \in \Theta} \operatorname{ker} \theta \neq\{0\}
$$

(ii) El subespacio libre de interacción (también llamado subespacio libre de decoherencia [36 [37]), se define mediante

$$
W_{D}:=\bigcap_{\omega \in \mathbf{B}_{+}}\left(\operatorname{ker} D_{\omega} \cap \operatorname{ker} D_{\omega}^{*}\right)
$$

Proposición 3.1. Sea $\mathcal{L}$ un generador de Markov de WCLT y $D \in \mathcal{B}(\mathrm{h})$ un operador de interacción. Entonces,

(i) $W_{D} \neq\{0\}$ si y sólo si el subconjunto

$$
\Theta:=\left\{P_{l} D P_{k}, P_{k} D^{*} P_{l}: l<k\right\}
$$

es singular.

(ii) $0 \neq u \in W_{D}$ si y sólo si $u=\sum_{l} P_{l} u=\sum_{l} u_{l}$, con

$$
u_{l} \in V_{l}:=\left(\bigcap_{k<l} \operatorname{ker}\left(P_{k} D P_{l}\right) \bigcap_{k>l} \operatorname{ker}\left(P_{k} D^{*} P_{l}\right)\right) \cap P_{l}(\mathbf{h})
$$

para cada l.

(iii) $V_{k} \perp V_{l}$ si $k \neq l$ y $W_{D}=\bigoplus_{l} V_{l}$.

Demostración. Basta probar que

$$
\operatorname{ker} D_{\omega}=\bigcap_{\left(\epsilon_{n}, \epsilon_{m}\right) \in B_{+, \omega}} \operatorname{ker}\left(P_{m} D P_{n}\right)
$$

para cada $\omega \in B_{+}$. 
Por definición

$$
D_{\omega}=\sum_{\left(\epsilon_{n}, \epsilon_{m}\right) \in B_{+, \omega}} P_{m} D P_{n}
$$

así que es suficiente probar la inclusión $\subset$. Por el lema 3.1 en [1], la condición $\left(\epsilon_{n}, \epsilon_{m}\right),\left(\epsilon_{n^{\prime}}, \epsilon_{m}\right) \in B_{+, \omega}$ implica que $\epsilon_{n}=\epsilon_{n^{\prime}}$. Por lo tanto, para cada $m$ tal que $\left(\epsilon_{n}, \epsilon_{m}\right) \in B_{+, \omega}$, la proyección espectral $P_{m}$ aparece en (3.3) a la izquierda de un sólo sumando. Esto implica que los operadores $P_{m} D P_{n}, \quad\left(\epsilon_{n}, \epsilon_{m}\right) \in B_{+, \omega}$ tienen rangos mutuamente ortogonales. Por consiguiente,

$$
0=D_{\omega} u=\sum_{\left(\epsilon_{n}, \epsilon_{m}\right) \in B_{+, \omega}} P_{m} D P_{n} u
$$

implica que $P_{m} D P_{n} u=0$ para cada par $\left(\epsilon_{n}, \epsilon_{m}\right) \in B_{+, \omega}$. La identidad (3.2) se sigue inmediatamente. De manera similar, usando el mismo lema, se verifica la identidad $\operatorname{ker} D_{\omega}^{*}=\bigcap_{\left(\epsilon_{n}, \epsilon_{m}\right) \in B_{+, \omega}} \operatorname{ker}\left(P_{n} D^{*} P_{m}\right)$. Esto prueba $(i)$. Ahora, cada $u \neq 0$ puede ser escrito en la forma $u=\sum_{l} u_{l}$, con $u_{l}=P_{l} u$. Supongamos que $u_{l} \in V_{l}=\cap_{k<l} \operatorname{ker}\left(P_{k} D P_{l}\right) \cap_{k>l} \operatorname{ker}\left(P_{k} D^{*} P_{l}\right) \cap P_{l}(\mathrm{~h})$, para cada $l \geq 0$. Entonces, si $m \neq l$ se obtiene que $P_{k} D P_{m} u_{l}=0$ y $P_{k} D^{*} P_{m} u_{l}=0$, puesto que $P_{m} u_{l}=0$ para cada $m \neq l$. Consecuentemente, $u_{l} \in W_{D}$ para cada $u_{l} \in V_{l}$. Por lo tanto, $u=\sum_{l} u_{l} \in W_{D}$. Recíprocamente, si $u \in W_{D}$, entonces $u_{l}=P_{l} u \in V_{l}$ para cada $l \geq 0$ dado que $u \in W_{D} \subset \cap_{k<l} \operatorname{ker}\left(P_{k} D P_{l}\right) \cap_{k>l} \operatorname{ker}\left(P_{k} D^{*} P_{l}\right)$. Por lo tanto, $u=$ $\sum_{l} u_{l}$ con cada $u_{l} \in V_{l}$. Esto prueba $(i i)$. El inciso $(i i i)$ se sigue inmediatamente de $(i i)$ y del hecho que las proyecciones espectrales $P_{l}$ tienen rangos mutuamente ortogonales. Esto termina la prueba.

Observación 3.2. Identificando cada sumando de los operadores de Kraus como la matriz $P_{m} D P_{n}, m<n$, con su único bloque no trivial de tamaño $r_{m} \times r_{n}$, donde $r_{l}=\operatorname{rango}\left(P_{l}\right)$, se obtiene la matriz autoadjunta

$$
\mathcal{D}_{B_{+}}:=\sum_{m<n}\left(P_{m} D P_{n}+P_{n} D^{*} P_{m}\right)
$$

como una matriz de bloques iguales a $P_{m} D P_{n}, m \neq n$ : 


$\left(\begin{array}{c|c|c|c|c|c|c}0 & P_{0} D P_{1} & P_{0} D P_{2} & P_{0} D P_{3} & \ldots & P_{0} D P_{k} & \cdots \\ \hline P_{1} D^{*} P_{0} & 0 & P_{1} D P_{2} & P_{1} D P_{3} & \ldots & P_{1} D P_{k} & \ldots \\ \hline P_{2} D^{*} P_{0} & P_{2} D^{*} P_{1} & 0 & P_{2} D P_{3} & \ldots & P_{2} D P_{k} & \ldots \\ \hline P_{3} D^{*} P_{0} & P_{3} D^{*} P_{1} & P_{3} D^{*} P_{2} & 0 & \ldots & P_{3} D P_{k} & \ldots \\ \hline \vdots & \vdots & \vdots & \vdots & 0 & \vdots & \ldots \\ \hline P_{k} D^{*} P_{0} & P_{k} D^{*} P_{1} & P_{k} D^{*} P_{2} & P_{k} D^{*} P_{3} & \ldots & 0 & \cdots \\ \hline \vdots & \vdots & \vdots & \vdots & \vdots & \vdots & \ddots\end{array}\right)$

Observación 3.3. Nótese que la matriz $\mathcal{D}_{B_{+}}$es autoadjunta, consecuentemente, los subespacios $V_{l}, \forall l \geq 0$, definidos en el inciso (ii) en la proposición 3.1] también pueden ser definidos como

$$
V_{l}:=\left(\bigcap_{k<l} \operatorname{ker}\left(P_{l} D^{*} P_{k}\right) \bigcap_{k>l} \operatorname{ker}\left(P_{l} D P_{k}\right)\right) \cap P_{l}(\mathrm{~h}) ; \quad \forall l \geq 0 .
$$

Teorema 3.1. Con las notaciones de la proposición anterior, sea $\mathcal{L}_{*}:=\mathcal{L}_{* 0}-i \delta_{\Delta}$ un generador de Markov de WCLT asociado con un Hamiltoniano de referencia $H \in \mathcal{B}(\mathrm{h})$ y Hamiltoniano efectivo $\Delta=\sum_{\omega} \Delta_{\omega}$. Sea $D \in \mathcal{B}(\mathrm{h})$ un operador de interacción satisfaciendo que $W_{D} \neq\{0\}$. Entonces, para cada estado

$$
\rho:=\sum_{j} \lambda_{j}\left|u_{j}\right\rangle\left\langle u_{j}\right|
$$

con $u_{j} \in V_{j} \subset W_{D},\left\|u_{j}\right\|=1$ y $\sum_{j} \lambda_{j}=1$, se satisface $\mathcal{L}_{* 0}(\rho)=0$. Además, si $\delta_{\Delta_{\omega}}(\rho)=\left[\Delta_{\omega}, \rho\right]$, donde

$$
\Delta_{\omega}=\zeta_{-, \omega} D_{\omega}^{*} D_{\omega}+\zeta_{+, \omega} D_{\omega} D_{\omega}^{*}
$$

$y \zeta_{-, \omega}, \zeta_{+, \omega} \in \mathbb{R}$, entonces $\rho$ es un estado estacionario.

Demostración. Para cada $u_{j} \in V_{j}$, el operador $\left|u_{j}\right\rangle\left\langle u_{j}\right|$ satisface $\mathcal{L}_{* 0}\left(\left|u_{j}\right\rangle\left\langle u_{j}\right|\right)=$ 0 , es decir, para cada $u_{j} \in V_{j}$, el operador $\left|u_{j}\right\rangle\left\langle u_{j}\right| \in \operatorname{ker}\left(\mathcal{L}_{* 0}\right)$. El generador $\mathcal{L}_{* 0}$ es un operador cerrado en el espacio de Banach de los operadores de traza finita en h. Siendo $\rho$ el límite de una sucesión de aproximaciones de rango finito $\rho_{n} \in \operatorname{dom}\left(\mathcal{L}_{* 0}\right)$ y la identidad $\mathcal{L}_{* 0}\left(\rho_{n}\right)=0$ para todo $n \geq 0$, implican que $\rho \in$ $\operatorname{dom}\left(\mathcal{L}_{* 0}\right)$ y $\mathcal{L}_{* 0}(\rho)=0$. Además, la condición $u_{j} \in W_{D}$ para cada $j$, implica que $\Delta_{\omega}$ conmuta con $\rho$. Esto termina la prueba. 
Observación 3.4. Para cada $0 \neq u_{j} \in V_{j}$, el operador $\rho_{u_{j}}:=P_{j} \rho P_{j}=\lambda_{j}\left|u_{j}\right\rangle\left\langle u_{j}\right|$ actúa de manera no trivial sólo sobre el subespacio soporte $P_{j}(\mathrm{~h})$ de la proyección ortogonal $P_{j}$. Por lo tanto, se puede identificar $\rho_{u_{j}}$ con su único bloque no trivial. Además, dado que $\mathrm{h}=\bigoplus_{j} P_{j}(\mathrm{~h})$, la identidad $\rho=\bigoplus_{j} \rho_{u_{j}}$ se satisface. Por lo tanto, el estado dado por (3.5) en el teorema 3.1 tiene la siguiente estructura matricial explícita,

$\rho=\frac{1}{\sum_{u_{j} \neq 0} \lambda_{j} \rho P_{0}}\left(\begin{array}{l|l|l|l|l|l} & & & & & \\ \hline & P_{1} \rho P_{1} & & & & \\ \hline & & P_{2} \rho P_{2} & & & \\ \hline & & & \ddots & P_{j} \rho P_{j} & \\ \hline & & & & & \ddots\end{array}\right)$

con cada $P_{j} \rho P_{j}=\lambda_{j}\left|u_{j}\right\rangle\left\langle u_{j}\right|$ un bloque de dimensión $r_{j} \times r_{j}$.

Observación 3.5. (i) Nótese que la condición $u \in W_{D}$, implica que el estado puro $|u\rangle\langle u|$ es un estado estacionario extremo. Además, este operador pertenece a la subálgebra de puntos fijos $\mathcal{F}(\mathcal{T})$, del semigrupo directo $\left(\mathcal{T}_{t}\right)_{t \geq 0}$.

(ii) En el caso cuando $\mathrm{h}$ tiene dimensión finita, se puede escribir $\mathcal{B}\left(\mathbb{C}^{d}\right)=$ $\{H\}^{\prime} \oplus\left(\{H\}^{\prime}\right)^{\perp}$, con respecto al producto interno Hilbert-Schmidt. Entonces, para cada $D \in \mathcal{B}\left(\mathbb{C}^{d}\right)$, existen únicos $D_{1} \in\{H\}^{\prime}$ y $D_{2} \in\left(\{H\}^{\prime}\right)^{\perp}$ tal que $D=D_{1}+D_{2}$. Por lo tanto, para cada frecuencia de Bohr $\omega:=$ $\epsilon_{n}-\epsilon_{m}>0$ se tiene que

$$
\begin{aligned}
D_{\omega} & :=\sum_{\left(\epsilon_{n}, \epsilon_{m}\right) \in \mathbf{B}_{+, \omega}} P_{m} D P_{n}=\sum_{\left(\epsilon_{n}, \epsilon_{m}\right) \in \mathbf{B}_{+, \omega}} P_{m}\left(D_{1}+D_{2}\right) P_{n} \\
& =\sum_{\left(\epsilon_{n}, \epsilon_{m}\right) \in \mathbf{B}_{+, \omega}} P_{m} D_{1} P_{n}+\sum_{\left(\epsilon_{n}, \epsilon_{m}\right) \in \mathbf{B}_{+, \omega}} P_{m} D_{2} P_{n} \\
& =\sum_{\left(\epsilon_{n}, \epsilon_{m}\right) \in \mathbf{B}_{+, \omega}} P_{m} D_{2} P_{n} .
\end{aligned}
$$


Esto implica que los operadores de interacción relevantes son aquellos que pertenecen a $\left(\{H\}^{\prime}\right)^{\perp}$. Más aún, la definición de los operadores de Kraus (1.2), implica que basta considerar a las matrices triangulares superiores (o triangulares inferiores) como operadores de interacción.

Observación 3.6. Obsérvese que $\left(\{H\}^{\prime}\right)^{\perp}$ es el aniquilador de $\{H\}^{\prime}$, dado que $\left(\{H\}^{\prime}\right)^{\perp}:=\left\{A \in \mathcal{B}\left(\mathbb{C}^{d}\right): \operatorname{tr}\left(B^{*} A\right)=0, \forall B \in\{H\}^{\prime}\right\}$.

El teorema siguiente caracteriza a los estados estacionarios soportados en $W_{D}$ en términos de su producto con los operadores de Kraus.

Teorema 3.2. Un estado estacionario $\rho$ tiene la forma (3.5) para algunos $u_{j} \in W_{D}$ y $\lambda_{j}>0$ si y sólo si

$$
\rho D_{\omega}=0=D_{\omega} \rho
$$

para cada frecuencia de Bohr $\omega$.

Demostración. Si $\rho$ tiene la forma (3.5) entonces inmediatamente se sigue que $\rho D_{\omega}=0=D_{\omega} \rho$, dado que el soporte de $\rho$ es un subconjunto de $W_{D}$.

Recíprocamente, sea $\rho$ un estado, entonces $\rho=\sum_{k} \rho_{k}\left|u_{k}\right\rangle\left\langle u_{k}\right|$ para algún sistema ortonormal completo $\left(u_{k}\right)$ de h y $\rho_{k}>0$. Además, si para cada frecuencia de Bohr $\rho D_{\omega}=0=D_{\omega} \rho$ entonces

$$
\sum_{k} \rho_{k}\left|D_{\omega} u_{k}\right\rangle\left\langle u_{k}\right|=D_{\omega} \rho=0 .
$$

Por lo tanto, para cada $u_{l}$ se tiene que

$$
0=\sum_{k} \rho_{k}\left|D_{\omega} u_{k}\right\rangle\left\langle u_{k}\right| u_{l}=\rho_{l} D_{\omega} u_{l}
$$

lo cual implica que $u_{l} \in \operatorname{ker} D_{\omega}$ para cada frecuencia de Bohr $\omega$. Igualmente, de $\rho D_{\omega}=0=D_{\omega}^{*} \rho$ se obtiene que

$$
0=D_{\omega}^{*} \rho=\sum_{k} \rho_{k}\left|D_{\omega}^{*} u_{k}\right\rangle\left\langle u_{k}\right|
$$

y para cada $u_{l}$, se implica que

$$
0=\sum_{k} \rho_{k}\left\langle u_{k}, u_{l}\right\rangle D_{\omega}^{*} u_{k}=\rho_{l} D_{\omega}^{*} u_{l} .
$$

Consecuentemente, $u_{l} \in \operatorname{ker} D_{\omega}^{*}$ para cada frecuencia de Bohr $\omega$. Se concluye que $u_{l} \in W_{D}$ para cada $l$. Por lo tanto, $\rho$ tiene la estructura (3.5). Esto termina la prueba. 


\subsection{Estados estacionarios de balance detallado local}

Los estados estacionarios de la forma (3.5) en el teorema 3.1, son los estados estacionarios más simples de generadores de Markov de WCLT, pero no todos los estados estacionaros pertenecen a esta clase. Por ejemplo, si el Hamiltoniano de referencia es no degenerado, existen estados de Gibbs o estados estacionarios localmente de Gibbs, que dependen de los coeficientes $\Gamma_{ \pm, \omega}$ 's (ver las secciones 2.2 y 2.3. Por otra parte, en el caso de un Hamiltoniano de referencia degenerado, los estados estacionarios exhiben una estructura matricial a bloques, dichos bloques pueden depender o no de los coeficientes $\Gamma_{ \pm, \omega}$ 's (ver sección 3.5 y ejemplos en la sección 3.4.

A continuación, se generaliza el teorema de la sección previa.

Teorema 3.3. Con las notaciones del teorema 3.1 sea $\mathcal{L}_{*}=\mathcal{L}_{* 0}-i \delta_{\Delta}$ un generador de Markov de WCLT asociado con H. Entonces,

(i) un estado $\rho$ conmuta con la proyección ortogonal sobre el subespacio $W_{D}$, $P_{W_{D}}$, si y sólo si $\rho$ tiene la estructura

$$
\rho=P_{W_{D}} \rho P_{W_{D}}+P_{W_{D}^{\perp}} \rho P_{W_{D}^{\perp}}
$$

donde $P_{W_{D}^{\perp}}$ es la proyección ortogonal sobre $W_{D}^{\perp}$.

(ii) Si además $\rho$ es estacionario, entonces

$$
\rho=\theta \rho_{W_{D}}+(1-\theta) \rho_{W_{D}^{\perp}}, \quad \theta \in[0,1]
$$

con $\rho_{W_{D}}$ un estado estacionario soportado en $W_{D}$ y $\rho_{W_{D}^{\perp}}$ un estado estacionario soportado en $W_{D}^{\perp}$.

Demostración. Dado que $P_{W_{D}}+P_{W_{D}^{\perp}}=1$, entonces para cualquier estado $\rho$,

$$
P_{W_{D}} \rho+P_{W_{D}^{\perp}} \rho=\rho=\rho P_{W_{D}}+\rho P_{W_{D}^{\perp}} .
$$

Por consiguiente, $\rho$ conmuta con $P_{W_{D}}$ si y sólo si conmuta con $P_{W_{D}^{\perp}}$.

La hipótesis $\rho$ conmuta con $P_{W_{D}}$ y cálculos simples muestran que

$$
\rho=\left(P_{W_{D}}+P_{W_{D}^{\perp}}\right) \rho\left(P_{W_{D}}+P_{W_{D}^{\perp}}\right)=P_{W_{D}} \rho P_{W_{D}}+P_{W_{D}^{\perp}} \rho P_{W_{D}^{\perp}} .
$$

Recíprocamente, si $\rho$ tiene la estructura 3.6, entonces los subespacios $W_{D}$ y $W_{D}^{\perp}$ son invariantes bajo la acción de $\rho$, consecuentemente, $\rho$ conmuta con la proyección ortogonal $P_{W_{D}}$. Esto prueba $(i)$. 
Si $\rho$ conmuta con $P_{W_{D}}$, donde $\rho=\sum_{j} \rho_{j}\left|u_{j}\right\rangle\left\langle u_{j}\right|$ para algunos $u_{j} \in \mathrm{h}, \rho_{j}>0$, entonces

$$
P_{W_{D}} \rho=P_{W_{D}} \rho P_{W_{D}}=\sum_{j} \rho_{j}\left|P_{W_{D}} u_{j}\right\rangle\left\langle P_{W_{D}} u_{j}\right|
$$

es invariante, dado que $W_{D} \subset \operatorname{ker} D_{\omega} \cap \operatorname{ker} D_{\omega}^{*}$, para cada frecuencia de Bohr $\omega$ y $\Delta_{\omega}=\zeta_{-, \omega} D_{\omega}^{*} D_{\omega}+\zeta_{+, \omega} D_{\omega} D_{\omega}^{*} \operatorname{con} \zeta_{-, \omega}, \zeta_{+, \omega} \in \mathbb{R}$, para cada frecuencia de Bohr $\omega$. Consecuentemente, si $\rho$ es invariante entonces

$$
P_{W_{D}^{\perp}} \rho P_{W_{D}^{\perp}}=\rho-P_{W_{D}} \rho P_{W_{D}}
$$

es invariante debido a la linealidad del generador infinitesimal $\mathcal{L}_{*}$. Además, el soporte de $P_{W_{D}} \rho P_{W_{D}}=P_{W_{D}} \rho$ es un subconjunto de $W_{D}$ y el soporte de $P_{W_{D}^{\perp}} \rho P_{W_{D}^{\perp}}=$ $P_{W_{D}^{\perp}} \rho$ es ortogonal al primero. Para completar la prueba de $(i i)$, sea

$$
\rho_{W_{D}}=\left(\sum_{j} \rho_{j}\left\|P_{W_{D}} u_{j}\right\|^{2}\right)^{-1} P_{W_{D}} \rho P_{W_{D}}
$$

$\mathrm{y}$

$$
\rho_{W_{D}^{\perp}}=\left(\sum_{j} \rho_{j}\left\|P_{W_{D}^{\perp}} u_{j}\right\|^{2}\right)^{-1} P_{W_{D}^{\perp}} \rho P_{W_{D}^{\perp}}
$$

Claramente estos operadores son estados estacionarios y mediante la normalización $\theta=\sum_{j} \rho_{j}\left\|P_{W_{D}} u_{j}\right\|^{2}$ se obtiene que

$$
\rho=\theta \rho_{W_{D}}+(1-\theta) \rho_{W_{D}^{\perp}}
$$

Esto termina la prueba.

Lema 3.1. $W_{D}=\bigcap_{\omega \in B_{+}} \operatorname{ker}\left(D_{\omega} D_{\omega}^{*}+D_{\omega}^{*} D_{\omega}\right)$.

Demostración. La inclusión $W_{D} \subset \bigcap_{\omega \in B_{+}} \operatorname{ker}\left(D_{\omega} D_{\omega}^{*}+D_{\omega}^{*} D_{\omega}\right)$ es inmediata. Ahora, si $D_{\omega} D_{\omega}^{*} u+D_{\omega}^{*} D_{\omega} u=0$, entonces la igualdad

$$
0=\left\langle u, D_{\omega} D_{\omega}^{*} u\right\rangle+\left\langle u, D_{\omega}^{*} D_{\omega} u\right\rangle=\left\|D_{\omega}^{*} u\right\|^{2}+\left\|D_{\omega} u\right\|^{2}
$$

implica que $\left\|D_{\omega}^{*} u\right\|^{2}=0$ y $\left\|D_{\omega} u\right\|^{2}=0$. Por lo tanto, $u \in \operatorname{ker} D_{\omega} \cap \operatorname{ker} D_{\omega}^{*}$. La inclusión $W_{D} \supset \bigcap_{\omega \in B_{+}} \operatorname{ker}\left(D_{\omega} D_{\omega}^{*}+D_{\omega}^{*} D_{\omega}\right)$ se sigue inmediatamente. Esto termina la prueba. 
Teorema 3.4. Sea $\mathcal{L}_{*}$ un generador de Markov de WCLT asociado con un Hamiltoniano de referencia $H$ y sea $\rho$ un estado. Si para cada frecuencia de Bohr $\omega$ se satisface la condición

$$
\rho D_{\omega}=c_{\omega} D_{\omega} \rho
$$

para alguna constante real $c_{\omega} \neq 0$ que puede depender de las frecuencias de Bohr, entonces,

(i) $\rho$ conmuta con $D_{\omega} D_{\omega}^{*} y D_{\omega}^{*} D_{\omega}$, para cada $\omega$,

(ii) $\rho$ tiene la estructura (3.6) en el teorema anterior,

(iii) si además, $c_{\omega}=\frac{\Gamma_{-, \omega}}{\Gamma_{+, \omega}}, \forall \omega$, entonces $\rho$ es un estado estacionario.

En particular, cualquier estado $\rho$ que satisfaga la condición 3.8$)$ con $c_{\omega}=\frac{\Gamma_{-, \omega}}{\Gamma_{+, \omega}}$, $\forall \omega$, pertenece al aniquilador de todos los operadores de Kraus.

Demostración. De (3.8), al tomar adjuntos se obtiene $\rho D_{\omega}^{*}=c_{\omega}^{-1} D_{\omega}^{*} \rho$, entonces

$$
\rho D_{\omega} D_{\omega}^{*}=c_{\omega} D_{\omega} \rho D_{\omega}^{*}=c_{\omega} D_{\omega} c_{\omega}^{-1} D_{\omega}^{*} \rho=D_{\omega} D_{\omega}^{*} \rho .
$$

Análogamente se prueba que $\rho D_{\omega}^{*} D_{\omega}=D_{\omega}^{*} D_{\omega} \rho$. Esto prueba $(i)$.

El inciso $(i)$ implica que $\rho$ conmuta con $D_{\omega} D_{\omega}^{*}+D_{\omega}^{*} D_{\omega}$ para cada frecuencia de Bohr $\omega$, entonces,

$$
\left(D_{\omega} D_{\omega}^{*}+D_{\omega}^{*} D_{\omega}\right) \rho u=\rho\left(D_{\omega} D_{\omega}^{*}+D_{\omega}^{*} D_{\omega}\right) u=0
$$

si $u \in \operatorname{ker}\left(D_{\omega} D_{\omega}^{*}+D_{\omega}^{*} D_{\omega}\right)$, probando que $\operatorname{ker}\left(D_{\omega} D_{\omega}^{*}+D_{\omega}^{*} D_{\omega}\right)$ es invariante bajo la acción de $\rho$ para cada frecuencia de Bohr $\omega$. Consecuentemente, del lema 3.1 se obtiene que $W_{D}$ es invariante bajo la acción de $\rho$. Además, puesto que $\rho$ es autoadjunto, se implica que $W_{D}^{\perp}$ también es invariante bajo la acción de $\rho$. En efecto, si $u \in W_{D}^{\perp}$, entonces

$$
\langle\rho u, v\rangle=\langle u, \rho v\rangle=0, \quad \forall v \in W_{D}
$$

prueba que $\rho u \in W_{D}^{\perp}$. Por lo tanto, se concluye que $\rho$ conmuta con la proyección ortogonal $P_{W_{D}}$, consecuentemente, del inciso $(i)$ en el teorema 3.3, $\rho$ tiene la estructura (3.6). Esto prueba $(i i)$. 
Para probar $(i i i)$, observe que para cualquier estado que satisface (3.8) con $c_{\omega}=$ $\frac{\Gamma_{-, \omega}}{\Gamma_{+, \omega}}$ para todo $\omega$, se verifica que

$$
\begin{aligned}
\mathcal{L}_{\omega *}(\rho)= & \left.-\frac{\Gamma_{-, \omega}}{2}\left\{D_{\omega}^{*} D_{\omega}, \rho\right\}+\Gamma_{+, \omega} D_{\omega}^{*} \rho D_{\omega}\right) \\
& +\left(-\frac{\Gamma_{+, \omega}}{2}\left\{D_{\omega} D_{\omega}^{*}, \rho\right\}+\Gamma_{-, \omega} D_{\omega} \rho D_{\omega}^{*}\right)-i\left[\Delta_{\omega}, \rho\right] \\
= & \left(-\Gamma_{-, \omega}+c_{\omega} \Gamma_{+, \omega}\right) D_{\omega}^{*} D_{\omega} \rho+\left(-\Gamma_{+, \omega}+c_{\omega}^{-1} \Gamma_{-, \omega}\right) \rho D_{\omega}^{*} D_{\omega} \\
& -i \rho\left(\zeta_{-, \omega} D_{\omega}^{*} D_{\omega}+\zeta_{+, \omega} D_{\omega} D_{\omega}^{*}-c_{\omega}^{-1} c_{\omega} \zeta_{-, \omega} D_{\omega}^{*} D_{\omega}\right. \\
& \left.-\zeta_{+, \omega} c_{\omega} c_{\omega}^{-1} D_{\omega} D_{\omega}^{*}\right)=0
\end{aligned}
$$

para cada frecuencia de Bohr $\omega$. Esto prueba que $\rho$ es invariante.

Finalmente, la desigualdad $\Gamma_{-, \omega}>\Gamma_{+, \omega}$ implica que $c_{\omega} \neq 1$ para todo $\omega$, entonces la condición $\rho D_{\omega}=c_{\omega} D_{\omega} \rho$ implica que $\operatorname{tr}\left(\rho D_{\omega}\right)=c_{\omega} \operatorname{tr}\left(D_{\omega} \rho\right)=c_{\omega} \operatorname{tr}\left(\rho D_{\omega}\right)$, lo que a su vez implica que $\operatorname{tr}\left(\rho D_{\omega}\right)=0$. Consecuentemente, $\rho$ pertenece al aniquilador de todos los operadores de Kraus. Esto termina la prueba.

Definición 3.2. (i) Cualquier estado $\mathcal{L}_{*}$-invariante satisfaciendo (3.8) se llamará estado invariante de balance detallado local.

(ii) Si además, $c_{\omega}=\frac{\Gamma_{-, \omega}}{\Gamma_{+, \omega}}, \forall \omega$, el estado $\mathcal{L}_{*}$-invariante será llamado estado invariante de balance detallado.

Observación 3.7. Cualquier estado invariante de balance detallado es de balance detallado local, no obstante, existen estados invariantes de balance detallado local que no son de balance detallado (ver teorema 2.3 y las referencias [16 29]).

Proposición 3.2. La proyección ortogonal $P_{W_{D}}$ sobre $W_{D}$, pertenece al conmutante de $H$. Consecuentemente, $P_{W_{D}^{\perp}} \in\{H\}^{\prime}$.

Demostración. Del lema 3.1 en [1], la condición $\left(\epsilon_{n}, \epsilon_{m}\right)=\left(\epsilon_{n^{\prime}}, \epsilon_{m}\right) \in B_{+, \omega}$ implica que $\epsilon_{n}=\epsilon_{n^{\prime}}$. Por lo tanto, cada proyección ortogonal $P_{\epsilon_{m}}$ aparece a la izquierda de un sólo sumando de (1.2). Además, ordenando el conjunto $B_{+, \omega}:=$ $\left\{\epsilon_{m}: \epsilon_{m}+\omega \in S p(H)\right\}$ se obtiene que $B_{+, \omega}=\left\{\epsilon_{0}(\omega)<\epsilon_{1}(\omega)<\cdots\right\}$. A partir de ahora, simplemente se escribirá $\epsilon_{k}$ en lugar de $\epsilon_{k}(\omega)$ y por consiguiente, se reescribe $D_{\omega}=\sum_{\epsilon_{m} \in B_{+, \omega}} P_{\epsilon_{m}} D P_{\epsilon_{m}+\omega}$. Mediante cálculos directos se obtiene 
que

$$
\begin{aligned}
D_{\omega} D_{\omega}^{*} & =\sum_{\epsilon_{m} \in B_{+, \omega}} \sum_{\epsilon_{m^{\prime}} \in B_{+, \omega}} P_{\epsilon_{m}} D P_{\epsilon_{m}+\omega} P_{\epsilon_{m^{\prime}}+\omega} D^{*} P_{\epsilon_{m^{\prime}}} \\
& =\sum_{\epsilon_{m} \in B_{+, \omega}} P_{\epsilon_{m}} D P_{\epsilon_{m}+\omega} D^{*} P_{\epsilon_{m}} .
\end{aligned}
$$

con cada proyección ortogonal $P_{\epsilon_{k}}$ apareciendo del lado derecho y del lado izquierdo a lo más en un sumando. Análogamente,

$$
D_{\omega}^{*} D_{\omega}=\sum_{\epsilon_{m} \in B_{+, \omega}} P_{\epsilon_{m}+\omega} D^{*} P_{\epsilon_{m}} D P_{\epsilon_{m}+\omega}
$$

con cada proyección ortogonal $P_{\epsilon_{k}+\omega}$ apareciendo del lado derecho y del lado izquierdo a lo más en un sumando.

Ahora, para cada $P_{\epsilon_{k}}$ y cualquier $\omega$ se obtiene $P_{\epsilon_{k}} D_{\omega} D_{\omega}^{*}=P_{\epsilon_{k}} D P_{\epsilon_{k}+\omega} D^{*} P_{\epsilon_{k}}$ si $\epsilon_{m}=\epsilon_{k}$ para algún $\epsilon_{m} \in B_{+, \omega}$ y $P_{\epsilon_{k}} D_{\omega} D_{\omega}^{*}=0$ en otro caso. De forma similar, $D_{\omega} D_{\omega}^{*} P_{\epsilon_{k}}=P_{\epsilon_{k}} D P_{\epsilon_{k}+\omega} D^{*} P_{\epsilon_{k}}$ si $\epsilon_{m}=\epsilon_{k}$ para algún $\epsilon_{m} \in B_{+, \omega}$ y $D_{\omega} D_{\omega}^{*} P_{\epsilon_{k}}=0$ en otro caso. Entonces, cada proyección ortogonal $P_{\epsilon_{k}}$ conmuta con $D_{\omega} D_{\omega}^{*}$, para cualquier $\omega$. Análogamente, se verifica que cada proyección ortogonal $P_{\epsilon_{k}}$ conmuta con $D_{\omega}^{*} D_{\omega}$, para cada $\omega$. En consecuencia, cada proyección ortogonal $P_{\epsilon_{k}}$ conmuta con $\left(D_{\omega} D_{\omega}^{*}+D_{\omega}^{*} D_{\omega}\right)$, para cada $\omega$, implicando que $\operatorname{ker}\left(D_{\omega} D_{\omega}^{*}+D_{\omega}^{*} D_{\omega}\right)$ es invariante bajo la acción de cada proyección ortogonal $P_{\epsilon_{k}}$, para cualquier $\omega$. Consecuentemente, por el lema 3.1. $W_{D}$ es invariante bajo la acción de $P_{\epsilon_{k}}$, para cada $\epsilon_{k}$. Además, puesto que $P_{\epsilon_{k}}$ es autoadjunto, se implica que $W_{D}^{\perp}$ también es invariante bajo la acción de $P_{\epsilon_{k}}$, para cada $\epsilon_{k}$. Por lo tanto, se concluye que la proyección ortogonal $P_{W_{D}}$ conmuta con cada proyección espectral $P_{\epsilon_{k}}$, consecuentemente, $P_{W_{D}} \in\{H\}^{\prime}$. Finalmente, la identidad $P_{W_{D}}+P_{W_{D}^{\perp}}=11$ implica que $P_{W_{D}^{\perp}} \in\{H\}^{\prime}$. Esto termina la prueba.

Observación 3.8. Como consecuencia de la proposición anterior, si $\rho \in\{H\}^{\prime}$, entonces se puede agregar el término $i\left[\Delta_{1}, \rho\right]$ (con $\Delta_{1}$ cualquier función real del Hamiltoniano de referencia $H$ ) al generador de Markov de WCLT, de tal forma que el estado $P_{W_{D}} \rho P_{W_{D}}$ sea invariante.

Observación 3.9. (Balance detallado cuántico) En el caso cuando un estado $\rho$ de balance detallado es fiel, la condición (3.8) produce una representación GKSL privilegiada de $\mathcal{L}$, en el sentido de la referencia [25]. Por lo tanto, en este caso, la condición de balance detallado implica la condición de balance detallado 
cuántica

$$
\mathcal{L}-\tilde{\mathcal{L}}=2 i[\Delta, \cdot]
$$

donde $\tilde{\mathcal{L}}$ es el generador de Markov 0-dual. Además, en este caso,

- El semigrupo 0-dual $\mathcal{T}$ es un semigrupo cuántico de Markov y

- El generador de Markov $\mathcal{L}$ conmuta con $\sigma_{-i}$, donde $\left(\sigma_{t}\right)$ es el grupo modular asociado $\sigma_{t}(x)=\rho^{i t} x \rho^{-i t}(\operatorname{ver}[25])$.

Observación 3.10. Como se mostró en el teorema 3.4 la condición (3.8) implica que el estado $\rho$ pertenece al Ann $(D)$. El recíproco no es verdadero, de hecho, tomando $H=\epsilon_{0}\left|e_{0}\right\rangle\left\langle e_{0}\right|+\epsilon_{1}\left(\left|e_{1}\right\rangle\left\langle e_{1}|+| e_{2}\right\rangle\left\langle e_{2}\right|\right)$ y $D=\left|e_{0}\right\rangle\left\langle e_{1}|+| e_{1}\right\rangle\left\langle e_{2}\right|$, se verifica que el estado $\rho=\frac{1}{2}\left|e_{0}\right\rangle\left\langle e_{0}\left|+\frac{1}{4}\right| e_{1}+e^{i \theta} e_{2}\right\rangle\left\langle e_{1}+e^{i \theta} e_{2}\right|$ pertenece al Ann $(D)$, pero no existe una constante real $c_{\omega}$ tal que $\rho D_{\omega}=c_{\omega} D_{\omega} \rho$, para toda $\omega$.

\subsection{Ejemplos}

Ejemplo 3.1. (H degenerado) Considere un Hamiltoniano de referencia con la propiedad siguiente: para cada frecuencia de Bohr $\omega$, existe un único par de valores propios $\left(\epsilon_{n}, \epsilon_{m}\right)$ tal que $\omega=\epsilon_{n}-\epsilon_{m}$ (si además $H$ es no-degenerado, $\mathcal{L}$ se llama genérico). Sea $\mathcal{L}$ un generador de Markov de WCLT asociado con H, entonces $D_{\omega}=P_{m} D P_{n}$ si $\omega=\epsilon_{n}-\epsilon_{m}$, con proyecciones espectrales $P_{k}$ no necesariamente de rango uno. Sea $\rho$ un estado invariante fiel perteneciente a $\{H\}^{\prime \prime} \subset\{H\}^{\prime}$, dado que para cada $\omega, \rho D_{\omega}=\frac{\rho_{m}}{\rho_{n}} D_{\omega} \rho$, la condición de balance detallado 3.8$)$ se satisface si y sólo si $\frac{\rho_{m}}{\rho_{n}}=\frac{\Gamma_{-, \omega}}{\Gamma_{+, \omega}}$ para cada $\omega$; consecuentemente, el estado es invariante y tiene la estructura de bloques dada en el teorema 3.4 . Además, esta condición produce el estado de Gibbs a bloques

$$
\rho=\frac{1}{Z} e^{-\beta(H) H}
$$

Ejemplo 3.2. (Estados oscuros en la fotosíntesis cuántica (Modelo de KV)) Los estados estacionarios de un generador de Markov que modela el fenómeno de la fotosíntesis, propuesto por Kozyrev y Volovich [34] en el contexto del límite estocástico de sistemas cuánticos abiertos degenerados, fue estudiado en [28]. A continuación, se demuestra que los llamados estados estacionarios oscuros de 
este modelo corresponden con los estados soportados en el subespacio libre de interacción.

En este caso, el Hamiltoniano de referencia tiene la descomposición espectral

$$
H=\epsilon_{0} P_{0}+\epsilon_{1} P_{1}+\epsilon_{2} P_{2},
$$

donde $P_{0}=\left|e_{0}\right\rangle\left\langle e_{0}\left|, P_{1}=\right| e_{1}\right\rangle\left\langle e_{1}\left|, P_{2}=\sum_{j=2}^{N}\right| e_{j}\right\rangle\left\langle e_{j}\right| y \epsilon_{2}>\epsilon_{1}>\epsilon_{0}=0 . E l$ conjunto de las frecuencias de Bohr es $B_{+}=\left\{\omega_{1}=\epsilon_{2}, \omega_{2}=\epsilon_{2}-\epsilon_{1}, \omega_{3}=\epsilon_{1}\right\}$. En el segundo nivel hay dos vectores de entrelazamiento máximo linealmente dependientes: el vector fotónico brillante $\chi=\sum_{j=2}^{N} e_{j}$ y el vector fonónico brillante $\Psi=e^{i \theta} \chi, \theta \in(0,2 \pi) \backslash\{\pi\}$, de manera que los correspondientes estados puros coinciden $|\chi\rangle\langle\chi|=| \Psi\rangle\langle\Psi|$.

Salvo algunas constantes, los operadores de interacción parcial son:

$$
\begin{aligned}
& D_{\omega_{1}}=\left|e_{0}\right\rangle\langle\chi|, \\
& D_{\omega_{2}}=\left|e_{1}\right\rangle\langle\Psi|, \\
& D_{\omega_{3}}=\left|e_{0}\right\rangle\left\langle e_{1}\right| .
\end{aligned}
$$

Por lo tanto, $W_{D}=\left\{e_{0}, e_{1}, \chi\right\}^{\perp}$. Consecuentemente, cualquier estado estacionario soportado en el subespacio libre de interacción $W_{D}$ es ortogonal al estado fotónico brillante $|\chi\rangle\langle\chi|$, así como también con los estados puros $\left|e_{0}\right\rangle\left\langle e_{0}\right| y$ $\left|e_{1}\right\rangle\left\langle e_{1}\right|$. Por tal motivo, es un estado oscuro global en el sentido de la referencia [34], es decir, es oscuro con respecto al generador completo $\mathcal{L}$. Este resultado coincide con el resultado encontrado en [28], donde se demostró que cualquier estado invariante tiene la descomposición espectral

$$
\rho=\lambda\left(r_{0} P_{0}+r_{1} P_{1}+\frac{1}{N-1}|\chi\rangle\langle\chi|\right)+\left(1-\lambda\left(r_{0}+r_{1}-1\right)\right) \rho_{W_{D}},
$$

donde $\rho_{W_{D}}$ es un estado oscuro, $r_{0}$ y $r_{1}$ son funciones de los coeficientes $\Gamma$ 's $y$, $0 \leq \lambda \leq\left(1+r_{0}+r_{1}\right)^{-1}$. De hecho, asumiendo que $\rho$ es invariante y definiendo

$$
\left(1-\lambda\left(r_{0}+r_{1}-1\right)\right) \rho_{W_{D}}:=\rho-\lambda\left(r_{0} P_{0}+r_{1} P_{1}+\frac{1}{N-1}|\chi\rangle\langle\chi|\right)
$$

se verifica que $\rho_{W_{D}}$ es:

- soportado en $W_{D}=\left\{e_{0}, e_{1}, \chi\right\}^{\perp}$ (sólo basta completar la diagonalización de $\rho)$, consecuentemente es invariante $y$,

- es ortogonal (con respecto al producto Hilbert-Schmidt) con $P_{0}, P_{1} y|\chi\rangle\langle\chi|$. Por lo tanto, es oscuro global. 


\subsection{Estados estacionarios del modelo de transporte cuántico de [28]}

En [28] los estados invariantes del modelo de transporte cuántico ahí descrito, se caracterizaron en términos de algunas condiciones, incluyendo relaciones de conmutación con ciertos operadores del modelo. En esta sección, se describe completamente el conjunto de todos los estados invariantes del modelo, incluyendo su parametrización en términos de estados soportados en el rango de $|Z|^{2}=Z^{*} Z$, donde $Z$ es el llamado operador de interferencia. Resulta que $Z$ juega el papel de un "operador de creación generalizado" enviando el segundo subespacio propio del Hamiltoniano de referencia $H$ en el tercero, esta analogía permite calcular todos los estados invariantes mediante un método simple y considerablemente menos cálculos que los realizados en la referencia [28]. Además, se identifica el subespacio de recurrencia rápida, se estudia la convergencia al equilibrio, se caracteriza el dominio de atracción de cualquier estado estacionario, se estudia el transporte de estados y la ganancia de energía.

La principal motivación de estudiar este tipo de modelos es tratar de comprender el proceso de la fotosíntesis. Es bien sabido que la conversión natural de la energía solar en la fotosíntesis es uno de los procesos biológicos más importantes en los que la transferencia de energía electrónica juega un papel decisivo. El objetivo de la fotosíntesis es producir energía biológica, la cual es generada en proporción directa a las reacciones fotoinducidas de separación de carga que ocurren en complejos centros de reacción. Más concretamente, el fascinante mecanismo de la fotosíntesis se inicia con absorción de luz (fotones), continúa con el transporte de energía y culmina con su almacenamiento en un centro de reacción. Por otra parte, dicho proceso ha atraído la atención de investigadores de diversas áreas: Física, Química, Biología y Matemáticas, entre otras, quienes han producido una importante cantidad de trabajo científico experimental o teórico enfocado a comprender, describir y modelar este complejo mecanismo.

Nota. Modelos de transporte cuántico y fotosíntesis fueron introducidos en [6. 34], y posteriormente estudiados en [28]. Es relevante mencionar que el modelo de transporte cuántico desarrollado en [28] es ligeramente diferente del modelo presentado en [6]. Explícitamente, las estructuras de los generadores asociados a la frecuencia $\omega_{3}=\epsilon_{3}$ son diferentes, en particular, la parte que involucra el Hamiltoniano efectivo. 


\subsubsection{El modelo de transporte cuántico}

El correspondiente generador de Markov $\mathcal{L}$, pertenece a la clase de generadores de Markov de WCLT y el Hamiltoniano de referencia que describe el modelo de transporte cuántico de [28], está dado por

$$
H=0 P_{0}+\epsilon_{1} P_{1}+\epsilon_{2} P_{2}+\epsilon_{3} P_{3}
$$

$\operatorname{con} P_{0}=\left|e_{0}\right\rangle\left\langle e_{0}\left|, P_{1}=\right| e_{1}\right\rangle\left\langle e_{1}\left|, P_{2}=\sum_{j=2}^{N}\right| e_{j}\right\rangle\left\langle e_{j}\left|, P_{3}=\sum_{j=N+1}^{N+M}\right| e_{j}\right\rangle\left\langle e_{j}\right|$, $M \leq N-1$ y los valores propios satisfacen las desigualdades $0<\epsilon_{3}<\epsilon_{2}<\epsilon_{1}$. El operador de interacción es

$$
D=\left|T e_{N+1}\right\rangle\left\langle T e_{1}|+Z+| T e_{0}\right\rangle\left\langle T e_{N-1}\right|
$$

donde $Z$ es un operador de interferencia realizando transiciones entre los niveles $\epsilon_{2}$ y $\epsilon_{3}$,

$$
Z: P_{2}(\mathrm{~h}) \mapsto P_{3}(\mathrm{~h})
$$

definido en términos de algunos coeficientes de interferencia $g_{a b}$ (ver observación 2.2 en [28]) como

$$
Z=\frac{1}{\sqrt{N-1}} \sum_{b=N+1}^{N+M} \sum_{a=2}^{N} g_{a b}|b\rangle\langle a|
$$

y $T=P_{0}+P_{1}+Z+Z^{*}$ un operador de transporte. Los estados de entrelazamiento máximo (maximally entangled) se definen, salvo normalización, como $|\psi\rangle\langle\psi|$ con $\psi=\sum_{a=2}^{N}|a\rangle$ y $\left|\psi^{\prime}\right\rangle\left\langle\psi^{\prime}\right|$ con $\psi^{\prime}=\sum_{b=N+1}^{N+M}|b\rangle$. Escribiendo simplemente $T_{a}$ en lugar de $T|a\rangle$, se verifica que $\sqrt{N-1} Z_{N-1}=\psi^{\prime}$ y $\sqrt{N-1} Z_{N+1}^{*}=\psi$.

De las desigualdades entre los valores propios, se verifica que existen seis frecuencias de Bohr: $\omega_{1}=\epsilon_{1}-\epsilon_{2}, \omega_{2}=\epsilon_{2}-\epsilon_{3}, \omega_{3}=\epsilon_{3}, \omega_{4}=\epsilon_{1}, \omega_{5}=\epsilon_{2}$, $\omega_{6}=\epsilon_{1}-\epsilon_{3}$. No obstante, sólo tres frecuencias de Bohr tienen asociados generadores de Markov no triviales, así que

$$
\mathcal{L}=\mathcal{L}_{\omega_{1}}+\mathcal{L}_{\omega_{2}}+\mathcal{L}_{\omega_{3}}
$$

y $\mathcal{L}_{\omega_{4}}=\mathcal{L}_{\omega_{5}}=\mathcal{L}_{\omega_{6}}=0$.

Observación 3.11. Las relaciones $T=T^{*}, Z^{2}=0$ y $Z^{* 2}=0$ se verifican inmediatamente. Además, mediante cálculos directos se verifica que $|Z|$ es una proyección ortogonal, $T P_{2} T=P_{3}=Z Z^{*}$ y $T P_{3} T=|Z|=Z^{*} Z$ (ver corolario 2.1 en [28]). 
Por otra parte, se tiene que

$$
\begin{aligned}
& D_{\omega_{1}}=\frac{1}{\sqrt{N-1}}|\psi\rangle\left\langle e_{1}\right|, \\
& D_{\omega_{2}}=Z \\
& D_{\omega_{3}}=\frac{1}{\sqrt{N-1}}\left|e_{0}\right\rangle\left\langle\psi^{\prime}\right| .
\end{aligned}
$$

De manera que los operadores de Kraus son,

$$
\begin{aligned}
& L_{1}^{\omega_{1}}=\sqrt{2 \Gamma_{R e,-, \omega_{1}}}|\psi\rangle\left\langle e_{1}\right| \\
& L_{1}^{\omega_{2}}=\sqrt{2(N-1) \Gamma_{R e,-, \omega_{2}} Z} \\
& L_{1}^{\omega_{3}}=\sqrt{2 \Gamma_{R e,-, \omega_{3}}}\left|e_{0}\right\rangle\left\langle\psi^{\prime}\right| \\
& \left.L_{2}^{\omega_{1}}=\sqrt{2 \Gamma_{R e,+, \omega_{1}} \mid} e_{1}\right\rangle\langle\psi| \\
& L_{2}^{\omega_{2}}=\sqrt{2(N-1) \Gamma_{R e,+, \omega_{2}}} Z^{*} \\
& L_{2}^{\omega_{3}}=0
\end{aligned}
$$

con todos los coeficientes $\Gamma_{R e, \pm, \omega_{l}}$ positivos, para cada $1 \leq l \leq 2, \Gamma_{R e,-, \omega_{3}}>0 \mathrm{y}$ $\Gamma_{R e,+, \omega_{3}}=0(\operatorname{ver}[28])$.

El Hamiltoniano efectivo está dado por:

$$
\begin{aligned}
H_{e f f} & =\Gamma_{I m,+, \omega_{1}}|\psi\rangle\left\langle\psi\left|-(N-1) \Gamma_{\text {Im },-, \omega_{1}}\right| e_{1}\right\rangle\left\langle e_{1}\right| \\
& +(N-1) \Gamma_{I m,+, \omega_{2}} P_{3}-(N-1) \Gamma_{I m,-, \omega_{2}}|Z| \\
& -\Gamma_{I m,-, \omega_{3}}\left|\psi^{\prime}\right\rangle\left\langle\psi^{\prime}\right|
\end{aligned}
$$

con todos los coeficientes $\Gamma_{I m, \pm, \omega_{l}}$ positivos, para cada $1 \leq l \leq 3$ (ver [28]).

Mediante cálculos directos, se verifica que

$W_{D}=\left\{e_{0}, e_{1}, \psi, \psi^{\prime}\right\}^{\perp} \cap \operatorname{ker} Z \cap \operatorname{ker} Z^{*}$

$$
=\left\{e_{0}, e_{1}, \psi, \psi^{\prime}\right\}^{\perp} \cap\left\{Z^{*} e_{j}: j=N+1, \cdots, N+M\right\}^{\perp} \cap\left\{Z e_{j}: j=2, \cdots, N\right\}^{\perp} .
$$

Además, los vectores $\psi$ y $Z^{*} \psi^{\prime}$ son puntos fijos de la proyección ortogonal $|Z|$. En efecto,

$$
|Z| \psi=Z^{*} Z \sqrt{N-1} Z^{*} e_{N+1}=\sqrt{N-1} Z^{*} e_{N+1}=\psi
$$

$\mathrm{y}$

$$
|Z| Z^{*} \psi^{\prime}=Z^{*} Z Z^{*} \psi^{\prime}=Z^{*} \psi^{\prime}
$$


donde se utilizaron las identidades $Z Z^{*}=P_{3}$ y $Z^{*} Z=|Z|$. Por consiguiente, se puede escribir

$$
\operatorname{Im}|Z|=\operatorname{span}\left\{\psi, Z^{*} \psi^{\prime}\right\} \oplus \operatorname{Im}|Z| \cap\left\{\psi, Z^{*} \psi^{\prime}\right\}^{\perp} .
$$

Asimismo, la identidad $P_{2}(\mathrm{~h})=\operatorname{Im}|Z| \oplus \operatorname{ker}|Z|$ permite la descomposición

$$
P_{2}(\mathrm{~h})=\operatorname{span}\left\{\psi, Z^{*} \psi^{\prime}\right\} \oplus\left(\operatorname{Im}|Z| \cap\left\{\psi, Z^{*} \psi^{\prime}\right\}^{\perp}\right) \oplus \operatorname{ker}|Z| .
$$

Análogamente, la definición de $\psi^{\prime}$ y $|Z| \psi=\psi$ implican que $\psi^{\prime}$ y $Z \psi$ son puntos fijos de $P_{3}$. Consecuentemente, se obtiene la descomposición

$$
P_{3}(\mathrm{~h})=\operatorname{span}\left\{\psi^{\prime}, Z \psi\right\} \oplus \operatorname{Im} P_{3} \cap\left\{\psi^{\prime}, Z \psi\right\}^{\perp} .
$$

\subsubsection{Proyecciones subarmónicas y estructura de estados esta- cionarios}

Definición 3.3. Un operador positivo a es subarmónico (resp. superarmónico, resp. armónico) para un semigrupo cuántico de Markov $\mathcal{T}=\left(\mathcal{T}_{t}\right)_{t \geq 0}$ si $\mathcal{T}_{t}(a) \geq a$ (resp. $\mathcal{T}_{t}(a) \leq$ a, resp. $\mathcal{T}_{t}(a)=a$ ), para todo $t \geq 0$.

Las proyecciones subarmónicas juegan un papel fundamental en el estudio de la irreducibilidad del semigrupo [22]. En particular, las proyecciones ortogonales sobre los subespacios soporte de estados invariantes son subarmónicas. Aunque el recíproco no es cierto, el conocimiento de la proyecciones subarmónicas proporciona información relevante acerca de los estados estacionarios. A continuación, se calculan las proyecciones armónicas y subarmónicas del modelo de transporte como primer paso en la caracterización de estados estacionarios.

Proposición 3.3. Cada proyección de rango uno $|w\rangle\langle w|$ con $w \in W_{D}$ es armónica para el semigrupo del modelo de transporte cuántico. Consecuentemente, $P_{W_{D}} y$ $P_{W_{D}^{\perp}}$ son por sí mismas proyecciones armónicas.

Demostración. Basta demostrar que cada proyección de rango uno $|w\rangle\langle w|$, con $w \in W_{D}$ conmuta con el Hamiltoniano efectivo $H_{\text {eff }}=H_{\omega_{1}}+H_{\omega_{2}}+H_{\omega_{3}}$. Pero claramente $|w\rangle\langle w|$ conmuta con $H_{\omega_{1}}$ pues $w \in\left\{e_{1}, \psi\right\}^{\perp}$. También, conmuta con $H_{\omega_{3}}$ pues $w \perp \psi^{\prime}$. Ahora, para cada $v \in P_{3}(\mathrm{~h})$, la igualdad $Z^{*} v=0$ implica que $v=P_{3} v=Z Z^{*} v=0$, por lo tanto, $\operatorname{ker} Z^{*}=\operatorname{ker} P_{3}$ y por consiguiente $P_{3} w=0$. Además, $\operatorname{ker}|Z|=\operatorname{ker} Z$. En efecto, si $|Z| u=0$, entonces 
$0=Z 0=Z|Z| u=Z Z^{*} Z u=P_{3} Z u=Z u$, es decir, $\operatorname{ker}|Z| \subset \operatorname{ker} Z$, la otra inclusión es evidente. Por lo tanto, $w \in \operatorname{ker}|Z|$. Esto implica que $|w\rangle\langle w|$ conmuta con $H_{\omega_{2}}$, consecuentemente, conmuta con $H_{\text {eff }}$. Se concluye que cada $|w\rangle\langle w|$ es armónica. Al ser $P_{W_{D}}$ una combinación lineal de proyecciones de rango uno como las anteriores, se implica que $P_{W_{D}}$ es armónica por sí misma. Además, puesto que $P_{W_{D}}+P_{W_{D}^{\perp}}=\mathbb{1}$ y aplicando la conservatividad del semigrupo, se concluye que

$$
\mathcal{T}_{t}\left(P_{W_{D}^{\perp}}\right)=\mathcal{T}_{t}(\mathbb{1 l})-\mathcal{T}_{t}\left(P_{W_{D}}\right)=\mathbb{1}-P_{W_{D}}=P_{W_{D}^{\perp}} .
$$

Esto termina la prueba.

Observación 3.12. Obsérvese que en la demostración anterior se verifica que $\operatorname{ker} Z^{*}=\operatorname{ker} P_{3} y \operatorname{ker}|Z|=\operatorname{ker} Z$. Consecuentemente, de (3.13) y (3.14) se verifica que

$$
W_{D}^{\perp}=\operatorname{span}\left\{e_{0}, e_{1}\right\} \oplus \operatorname{Im}|Z| \oplus \operatorname{Im} P_{3} .
$$

Además de las proyecciones descritas previamente, el correspondiente semigrupo del modelo de transporte cuántico tiene otras proyecciones subarmónicas, como se muestra a continuación.

Proposición 3.4. La proyección ortogonal $p_{V}$ sobre el subespacio $V=\left\{e_{0}, e_{1}, \psi\right.$, $\left.\psi^{\prime}, Z \psi, Z^{*} \psi^{\prime}\right\}^{\perp}$ es subarmónica para el semigrupo cuántico de Markov del modelo de transporte cuántico.

Demostración. Por el teorema III.1 en [22], basta demostrar que la proyección ortogonal $p_{V}$ satisface que $\operatorname{Ran}\left(p_{V}\right)$ es invariante bajo la acción del semigrupo generado por el Hamiltoniano efectivo y satisface

$$
L_{k}^{\omega_{j}} u=p_{V} L_{k}^{\omega_{j}} u, \quad 1 \leq j \leq 3, \quad 1 \leq k \leq 2, \quad \forall u \in \operatorname{Ran}\left(p_{V}\right) .
$$

Estas condiciones son verdaderas si y sólo si $V=\operatorname{Ran}\left(p_{V}\right)$ es invariante bajo la acción del semigrupo generado por el Hamiltoniano efectivo $H_{e f f}=H_{\omega_{1}}+H_{\omega_{2}}+$ $H_{\omega_{3}}$ y las relaciones siguientes son válidas para todo $u \in V$ :

(i) $\left\langle e_{1}, u\right\rangle \psi=\left\langle e_{1}, u\right\rangle p_{V} \psi$,

(ii) $\langle\psi, u\rangle e_{1}=\langle\psi, u\rangle p_{V} e_{1}$,

(iii) $p_{V} Z u=Z u$,

(iv) $p_{V} Z^{*} u=Z^{*} u$, 
(v) $\left\langle\psi^{\prime}, u\right\rangle e_{0}=\left\langle\psi^{\prime}, u\right\rangle p_{V} e_{0}$.

De la forma explícita del Hamiltoniano efectivo $H_{\text {eff }}=H_{\omega_{1}}+H_{\omega_{2}}+H_{\omega_{3}}$, resulta que las condiciones $(i)-(v)$ implican que $V$ es invariante bajo la acción del grupo unitario generado por este operador. De hecho, la única parte no trivial por demostrar es que $p_{V}$ conmuta con

$$
H_{\omega_{2}}=(N-1) \Gamma_{I m,+, \omega_{2}} T P_{2} T-(N-1) \Gamma_{I m,-, \omega_{2}} T P_{3} T .
$$

Pero $T P_{2} T=P_{3}, T P_{3} T=|Z|$ y para $u \in V$ se obtiene que $P_{3} u=Z Z^{*} u=$ $Z p_{V} Z^{*} u=p_{V} Z p_{V} Z^{*} u=p_{V} Z Z^{*} u=p_{V} P_{3} u$, por consiguiente $P_{3} p_{V}=p_{V} P_{3}$. Análogamente, para $u \in V$, usando que $|Z|$ es una proyección se tiene que $|Z| u=$ $Z^{*} Z u=Z^{*} p_{V} Z u=p_{V} Z^{*} p_{V} Z u=p_{V}|Z| u$, es decir, $|Z| p_{V}=p_{V}|Z|$.

Ahora, si $p_{V}$ es la proyección ortogonal sobre el subespacio $V$, las condiciones $(i),(i i),(v)$ se verifican fácilmente para cada $u \in V$. Para demostrar que las condiciones $(i i i)-(i v)$ son válidas, basta probar que $Z u, Z^{*} u \in V$ para cada $u \in V$. En efecto, para cada $u \in V$, se sigue inmediatamente que $Z u, Z^{*} u \in\left\{e_{0}, e_{1}, \psi, \psi^{\prime}\right\}^{\perp}$. Además, note que $\langle Z u, Z \psi\rangle=\left\langle u,|Z|^{2} \psi\right\rangle=\langle u, \psi\rangle=0$ dado que $|Z|^{2}=|Z|$ es una proyección ortogonal y $\psi$ es punto fijo de $|Z|$ (es decir, $|Z| \psi=\psi$ ) y, $\left\langle Z u, Z^{*} \psi^{\prime}\right\rangle=\left\langle Z^{2} u, \psi^{\prime}\right\rangle=0$, ya que $Z^{2}=0$, por lo tanto, $Z u \in V$. Finalmente, $Z^{2}=0$ implica que $\left\langle Z^{*} u, Z \psi\right\rangle=\left\langle u, Z^{2} \psi\right\rangle=0 \mathrm{y}\left\langle Z^{*} u, Z^{*} \psi^{\prime}\right\rangle=\left\langle u, Z Z^{*} \psi^{\prime}\right\rangle=$ $\left\langle u, P_{3} \psi^{\prime}\right\rangle=\left\langle u, \psi^{\prime}\right\rangle=0$, esto muestra que $Z^{*} u \in V$ y termina la prueba.

\subsubsection{Estados invariantes}

En la presente sección se buscan estados invariantes asociados con las proyecciones subarmónicas no triviales. Como consecuencia del teorema 3.1. cada estado soportado en un subespacio de $W_{D}$ es necesariamente invariante. La existencia de estados invariantes con soporte contenido en $W_{D}^{\perp}$ es una pregunta que surge de forma natural. Para responder a esta pregunta se comienza con lo siguiente.

Lema 3.2. Sea $\rho$ un estado soportado en un subespacio de $V \cap W_{D}^{\perp}$. Entonces, $\rho$ es invariante si y sólo si $\left\{e_{1}, \psi, \psi^{\prime}\right\} \subset \operatorname{ker} \rho$, $\rho$ pertenece al conmutante del Hamiltoniano de referencia $\{H\}^{\prime}$, conmuta con $|Z|$ y satisface

$$
Z^{*} \rho Z=\frac{\Gamma_{R e,-, \omega_{2}}}{\Gamma_{R e,+, \omega_{2}}} \rho|Z|
$$

Demostración. Sea $\rho$ un estado soportado en un subespacio de $V \cap W_{D}^{\perp}$, es decir, Ran $\rho \subseteq V \cap W_{D}^{\perp} \subseteq V$, lo cual implica que $V^{\perp}=\left\{e_{0}, e_{1}, \psi, \psi^{\prime}, Z \psi, Z^{*} \psi^{\prime}\right\} \subseteq$ 
$(\operatorname{Ran} \rho)^{\perp}=\operatorname{ker} \rho$. Por lo tanto,

$$
\left\{e_{1}, \psi, \psi^{\prime}\right\} \subset \operatorname{ker} \rho
$$

Consecuentemente,

$$
\rho \in \operatorname{ker} \mathcal{L}_{\omega_{1 *}} \cap \operatorname{ker} \mathcal{L}_{\omega_{3^{*}}} .
$$

Por lo tanto, si $\rho$ es invariante, entonces

$$
\begin{aligned}
0=\mathcal{L}_{\omega_{2^{*}}}(\rho)= & -i\left(\Gamma_{\text {Im, }, \omega_{2}} P_{3} \rho-\Gamma_{I m,-, \omega_{2}}|Z| \rho\right) \\
& +i\left(\Gamma_{I m,+, \omega_{2}} \rho P_{3}-\Gamma_{I m,-, \omega_{2}} \rho|Z|\right) \\
& -\Gamma_{R e,-, \omega_{2}}\left(|Z| \rho-2 Z \rho Z^{*}+\rho|Z|\right) \\
& -\Gamma_{R e,+, \omega_{2}}\left(P_{3} \rho-2 Z^{*} \rho Z+\rho P_{3}\right) .
\end{aligned}
$$

Mediante cálculos directos, usando que $|Z|$ es una subproyección de $P_{2}$ (es decir, $\left.|Z| \leq P_{2}\right), P_{2} Z=0, Z^{*} P_{3}=0$ y $P_{2}=|Z|+\left(P_{2}-|Z|\right)$ se implica que

$$
\begin{aligned}
0=P_{2} \mathcal{L}_{\omega_{2^{*}}}(\rho) P_{3}= & -i\left(-\Gamma_{\text {Im },-, \omega_{2}}|Z| \rho P_{3}\right)-\Gamma_{R e,-, \omega_{2}}\left(|Z| \rho P_{3}\right) \\
& +i\left(\Gamma_{I m,+, \omega_{2}} P_{2} \rho P_{3}\right)-\Gamma_{R e,+, \omega_{2}}\left(P_{2} \rho P_{3}\right) \\
= & \left(-\Gamma_{R e,-, \omega_{2}}+i \Gamma_{I m,-, \omega_{2}}-\Gamma_{R e,+, \omega_{2}}+i \Gamma_{I m,+, \omega_{2}}\right)|Z| \rho P_{3} \\
& +\left(-\Gamma_{R e,+, \omega_{2}}+i \Gamma_{I m,+, \omega_{2}}\right)\left(P_{2}-|Z|\right) \rho P_{3} .
\end{aligned}
$$

La positividad de los coeficientes $\Gamma$ 's y la ortogonalidad de los rangos implican que $|Z| \rho P_{3}=0$ y $\left(P_{2}-|Z|\right) \rho P_{3}=0$. Por consiguiente, $P_{2} \rho P_{3}=0$ y tomando adjuntos se verifica que $P_{3} \rho P_{2}=0$. Posteriormente, utilizando que $\rho$ está soportado en un subespacio de $V \cap W_{D}^{\perp} \subset\left(P_{2}+P_{3}\right)($ h) se obtiene

$$
\begin{aligned}
{\left[\rho, P_{3}\right] } & =\left(P_{2}+P_{3}\right)\left[\rho, P_{3}\right]\left(P_{2}+P_{3}\right) \\
& =\left(P_{2}+P_{3}\right)\left(\rho P_{3}-P_{3} \rho\right)\left(P_{2}+P_{3}\right) \\
& =\left(P_{2} \rho P_{3}+P_{3} \rho P_{3}-P_{3} \rho\right)\left(P_{2}+P_{3}\right) \\
& =P_{2} \rho P_{3}-P_{3} \rho P_{2}=0 .
\end{aligned}
$$

Por lo tanto, $\rho$ conmuta con $P_{3}$. 
Por otra parte, usando la conmutatividad de $\rho$ con $P_{3}$, de (3.16) se obtiene que

$$
\begin{aligned}
0=\mathcal{L}_{\omega_{2^{*}}}(\rho)= & i \Gamma_{\text {Im },-, \omega_{2}}(|Z| \rho-\rho|Z|)-\Gamma_{R e,-, \omega_{2}}\left(|Z| \rho-2 Z \rho Z^{*}+\rho|Z|\right) \\
& +2 \Gamma_{R e,+, \omega_{2}}\left(Z^{*} \rho Z-P_{3} \rho\right) .
\end{aligned}
$$

Multiplicando por $P_{3}$ ambos lados de la ecuación anterior y utilizando nuevamente la conmutatividad de $\rho$ con $P_{3}$, se implica que

$$
0=P_{3} \mathcal{L}_{\omega_{2^{*}}}(\rho) P_{3}=\Gamma_{R e,-, \omega_{2}} Z \rho Z^{*}-\Gamma_{R e,+, \omega_{2}} P_{3} \rho .
$$

Por consiguiente,

$$
Z \rho Z^{*}=\frac{\Gamma_{R e,+, \omega_{2}}}{\Gamma_{R e,-, \omega_{2}}} P_{3} \rho .
$$

Multiplicando (3.18) por $Z^{*}$ a la izquierda y $Z$ a la derecha, es decir, $Z^{*} Z \rho Z^{*} Z=$ $\frac{\Gamma_{R e,+, \omega_{2}}}{\Gamma_{R e,-, \omega_{2}}} Z^{*} P_{3} \rho Z$ y usando que $|Z|=Z^{*} Z$ se obtiene que

$$
Z^{*} \rho Z=\frac{\Gamma_{R e,-, \omega_{2}}}{\Gamma_{R e,+, \omega_{2}}}|Z| \rho|Z| .
$$

Para probar (3.15) resta demostrar que $\rho$ conmuta con $|Z|$.

De (3.17) y usando (3.19) se obtiene que

$$
\begin{aligned}
0= & P_{2} \mathcal{L}_{\omega_{2^{*}}}(\rho) P_{2} \\
= & i \Gamma_{I m,-, \omega_{2}}\left(|Z| \rho P_{2}-P_{2} \rho|Z|\right)-\Gamma_{R e,-, \omega_{2}}\left(|Z| \rho P_{2}+P_{2} \rho|Z|\right)+2 \Gamma_{R e,+, \omega_{2}} Z^{*} \rho Z \\
= & i \Gamma_{I m,-, \omega_{2}}\left(|Z| \rho P_{2}-P_{2} \rho|Z|\right)-\Gamma_{R e,-, \omega_{2}}\left(|Z| \rho P_{2}+P_{2} \rho|Z|\right)+2 \Gamma_{R e,-, \omega_{2}}|Z| \rho|Z| \\
= & -\Gamma_{R e,-, \omega_{2}}|Z| \rho\left(P_{2}-|Z|\right)-\Gamma_{R e,-, \omega_{2}}\left(P_{2}-|Z|\right) \rho|Z| \\
& +i \Gamma_{\text {Im, }, \omega_{2}}|Z| \rho\left(P_{2}-|Z|\right)-i \Gamma_{I m,-\omega_{2}}\left(P_{2}-|Z|\right) \rho|Z| .
\end{aligned}
$$

Una vez más, de la positividad de los coeficientes $\Gamma$ 's y de la ortogonalidad de los rangos se obtiene que $|Z| \rho\left(P_{2}-|Z|\right)=0$ (equivalentemente, $|Z| \rho P_{2}=|Z| \rho|Z|$ ) y $\left(P_{2}-|Z|\right) \rho|Z|=0$ (equivalentemente, $P_{2} \rho|Z|=|Z| \rho|Z|$ ). Consecuentemente, $|Z| \rho P_{2}=|Z| \rho|Z|=P_{2} \rho|Z|$. Posteriormente, usando que $\left(P_{2}-|Z|\right) \leq P_{2}$ y $\left[\rho, P_{3}\right]=0$ se obtiene

$$
\begin{aligned}
{\left[\rho, P_{2}-|Z|\right] } & =\left(P_{2}+P_{3}\right)\left[\rho, P_{2}-|Z|\right]\left(P_{2}+P_{3}\right) \\
& =\left(P_{2}+P_{3}\right)\left(\rho\left(P_{2}-|Z|\right)-\left(P_{2}-|Z|\right) \rho\right)\left(P_{2}+P_{3}\right) \\
& =P_{2} \rho\left(P_{2}-|Z|\right)-\left(P_{2}-|Z|\right) \rho P_{2} \\
& =|Z| \rho P_{2}-P_{2} \rho|Z|=0 .
\end{aligned}
$$


Esto demuestra que $\rho$ conmuta con $P_{2}-|Z|$. De forma similar, usando que $\rho$ conmuta con $P_{3}$, se obtiene que

$$
\begin{aligned}
{[\rho,|Z|] } & =\left(P_{2}+P_{3}\right)[\rho,|Z|]\left(P_{2}+P_{3}\right) \\
& =\left(P_{2}+P_{3}\right)(\rho|Z|-|Z| \rho)\left(P_{2}+P_{3}\right) \\
& =P_{2} \rho|Z|-|Z| \rho P_{2}=0 .
\end{aligned}
$$

Por lo tanto, $\rho$ conmuta con $P_{2}$ y con $|Z|$. La conmutación de $\rho$ con $P_{0}$ y $P_{1}$ son inmediatas. Por lo tanto, se concluye que $\rho \in\{H\}^{\prime}$.

Recíprocamente, si $\rho$ es un estado soportado en un subespacio de $V \cap W_{D}^{\perp}$, perteneciendo a $\{H\}^{\prime}$, conmutando con $|Z|$ y satisfaciendo (3.19), entonces $\rho \in$ $\operatorname{ker} \mathcal{L}_{\omega_{1^{*}}} \cap \operatorname{ker} \mathcal{L}_{\omega_{2^{*}}} \cap \operatorname{ker} \mathcal{L}_{\omega_{3^{*}}}$. Esto termina la prueba.

En seguida, se caracteriza el conjunto de estados invariantes del modelo de transporte cuántico, soportados en un subespacio de $V \cap W_{D}^{\perp}$.

Teorema 3.5. Sea $\rho$ un estado soportado en un subespacio de $V \cap W_{D}^{\perp}$. Entonces, $\rho$ es invariante para el modelo de transporte cuántico si y sólo si tiene la estructura

$$
\rho=\frac{\Gamma_{R e,+, \omega_{2}}}{\Gamma_{R e,+, \omega_{2}}+\Gamma_{R e,-, \omega_{2}}} \sigma+\frac{\Gamma_{R e,-, \omega_{2}}}{\Gamma_{R e,+, \omega_{2}}+\Gamma_{R e,-, \omega_{2}}} Z \sigma Z^{*},
$$

donde $\sigma: \operatorname{Im}|Z| \mapsto \operatorname{Im}|Z|$ es un estado soportado en un subespacio de $V \cap$ $\operatorname{Im}|Z|$.

Demostración. Como consecuencia del lema 3.2. cualquier estado invariante $\rho$ soportado en $V \cap W_{D}^{\perp}$ pertenece a $\{H,|Z|\}^{\prime}$ y, puede escribirse en la forma

$$
\begin{aligned}
\rho & =\left(|Z|+P_{3}\right) \rho\left(|Z|+P_{3}\right)=|Z| \rho|Z|+P_{3} \rho P_{3}=|Z| \rho|Z|+P_{3} \rho \\
& =|Z| \rho|Z|+\frac{\Gamma_{R e,-, \omega_{2}}}{\Gamma_{R e,+, \omega_{2}}} Z \rho Z^{*} \\
& =|Z| \rho|Z|+\frac{\Gamma_{R e,-, \omega_{2}}}{\Gamma_{R e,+, \omega_{2}}} P_{3} Z \rho Z^{*} P_{3} \\
& =|Z| \rho|Z|+\frac{\Gamma_{R e,-, \omega_{2}}}{\Gamma_{R e,+, \omega_{2}}} Z Z^{*} Z \rho Z^{*} Z Z^{*} \\
& =|Z| \rho|Z|+\frac{\Gamma_{R e,-, \omega_{2}}}{\Gamma_{R e,+, \omega_{2}}} Z|Z| \rho|Z| Z^{*}
\end{aligned}
$$




$$
\begin{aligned}
& =|Z| \rho|Z|+\frac{\Gamma_{R e,-, \omega_{2}}}{\Gamma_{R e,+, \omega_{2}}+\Gamma_{R e,-, \omega_{2}}} Z\left(\frac{\Gamma_{R e,+, \omega_{2}}+\Gamma_{R e,-, \omega_{2}}}{\Gamma_{R e,+, \omega_{2}}}|Z| \rho|Z|\right) Z^{*} \\
& =\frac{\Gamma_{R e,+, \omega_{2}}}{\Gamma_{R e,+, \omega_{2}}+\Gamma_{R e,-, \omega_{2}}} \sigma+\frac{\Gamma_{R e,-, \omega_{2}}}{\Gamma_{R e,+, \omega_{2}}+\Gamma_{R e,-, \omega_{2}}} Z \sigma Z^{*},
\end{aligned}
$$

donde $\sigma=\frac{\Gamma_{R e,+, \omega_{2}}+\Gamma_{R e,-, \omega_{2}}}{\Gamma_{R e,+, \omega_{2}}}|Z| \rho|Z|, Z \rho Z^{*}=P_{3} Z \rho Z^{*} P_{3}$ y se ha utilizado 3.18 . Claramente, salvo normalización, $\sigma: \operatorname{Im}|Z| \mapsto \operatorname{Im}|Z|$ es un estado soportado en un subespacio de $V \cap \operatorname{Im}|Z|$, puesto que $\rho$ está soportado en $V \cap W_{D}^{\perp}$.

Recíprocamente, si $\rho$ es de la forma (3.20), entonces $e_{1} \in \operatorname{ker} \rho$ y $\rho \psi=$ $\frac{\Gamma_{R e,+, \omega_{2}}}{\Gamma_{R e,+, \omega_{2}}+\Gamma_{R e,-, \omega_{2}}} \sigma \psi$, entonces para cualquier $u \in \mathrm{h}$ se implica que $\langle u, \sigma \psi\rangle=$ $\langle\sigma u, \psi\rangle=0$ dado que $\sigma u \in V \cap \operatorname{Im}|Z|$, por lo tanto $\sigma \psi=0$, probando que $\psi \in \operatorname{ker} \rho$. De forma similar, se prueba que $\psi^{\prime} \in \operatorname{ker} \rho$. Consecuentemente, $\rho \in \operatorname{ker} \mathcal{L}_{\omega_{1 *}} \cap \operatorname{ker} \mathcal{L}_{\omega_{3^{*}}}$. Por otra parte, mediante cálculos directos se verifica que $\rho$ conmuta con $H$ y con $|Z|$. En efecto, $|Z| \leq P_{2}$ implica que $\sigma$ conmuta con $P_{2}$ y usando $P_{2} Z=0=Z^{*} P_{2}, P_{3} Z \sigma Z^{*}=Z \sigma Z^{*}=Z \sigma Z^{*} P_{3}$, entonces

$$
\begin{aligned}
& P_{2} \rho=\frac{\Gamma_{R e,+, \omega_{2}}}{\Gamma_{R e,+, \omega_{2}}+\Gamma_{R e,-, \omega_{2}}} P_{2} \sigma=\rho P_{2}, \quad y \\
& P_{3} \rho=\frac{\Gamma_{R e,-, \omega_{2}}}{\Gamma_{R e,+, \omega_{2}}+\Gamma_{R e,-, \omega_{2}}} Z \sigma Z^{*}=\rho P_{3} .
\end{aligned}
$$

La conmutación de $\rho$ con $P_{0}$ y $P_{1}$ son inmediatas. La conmutación de $\rho$ con $|Z|$ se sigue de

$$
|Z| \rho=\frac{\Gamma_{R e,+, \omega_{2}}}{\Gamma_{R e,+, \omega_{2}}+\Gamma_{R e,-, \omega_{2}}} \sigma=\rho|Z| .
$$

Resta verificar (3.15), de $[\rho,|Z|]=0$ y $Z^{*}|Z|=0=|Z| Z$, se implica que

$$
\begin{aligned}
Z^{*} \rho Z & =Z^{*}\left(\frac{\Gamma_{R e,+, \omega_{2}}}{\Gamma_{R e,+, \omega_{2}}+\Gamma_{R e,-, \omega_{2}}} \sigma+\frac{\Gamma_{R e,-, \omega_{2}}}{\Gamma_{R e,+, \omega_{2}}+\Gamma_{R e,-, \omega_{2}}} Z Z^{*}\right) Z \\
& =\frac{\Gamma_{R e,-, \omega_{2}}}{\Gamma_{R e,+, \omega_{2}}+\Gamma_{R e,-, \omega_{2}}} Z^{*} Z \sigma Z^{*} Z \\
& =\frac{\Gamma_{R e,-, \omega_{2}}}{\Gamma_{R e,+, \omega_{2}}+\Gamma_{R e,-, \omega_{2}}}|Z|\left(\frac{\Gamma_{R e,+, \omega_{2}}+\Gamma_{R e,-, \omega_{2}}}{\Gamma_{R e,+, \omega_{2}}}|Z| \rho|Z|\right)|Z| \\
& =\frac{\Gamma_{R e,-, \omega_{2}}}{\Gamma_{R e,+, \omega_{2}}} \rho|Z| .
\end{aligned}
$$


Por lo tanto, el lema 3.2 implica que $\rho$ es invariante. Además, $\rho$ está soportado en $V \cap W_{D}^{\perp}$. Esto termina la prueba.

Observación 3.13. Recordemos que cualquier estado $\rho$ soportado en un subespacio de $W_{D}$ es invariante. Además, la dinámica de los estados soportados en $W_{D}$ es intrascendente o trivial. Entonces, como consecuencia del teorema anterior, los estados que se escriben como una combinación convexa de dos estados, explícitamente,

$$
\tau=\lambda \tau_{W_{D}}+(1-\lambda) \tau_{V \cap W_{D}^{\perp}}, \quad 0 \leq \lambda \leq 1,
$$

$\operatorname{con} \tau_{W_{D}}$ un estado soportado en $W_{D} y$

$$
\tau_{V \cap W_{D}^{\perp}}=\frac{\Gamma_{R e,+, \omega_{2}}}{\Gamma_{R e,+, \omega_{2}}+\Gamma_{R e,-, \omega_{2}}} \sigma+\frac{\Gamma_{R e,-, \omega_{2}}}{\Gamma_{R e,+, \omega_{2}}+\Gamma_{R e,-, \omega_{2}}} Z \sigma Z^{*},
$$

son invariantes para el modelo de transporte cuántico. Además, el soporte de los estados $\tau$ descritos por la ecuación 3.21] es $V$.

El resultado siguiente describe los puntos extremos del subconjunto de estados invariantes que tienen la estructura (3.21).

Teorema 3.6. Un estado invariante $\rho$ de la forma (3.21) es extremo para el modelo de transporte cuántico si y sólo si tiene una de las estructuras siguientes:

(i) $\rho=|u\rangle\langle u|$ con $u \in W_{D}=\left\{e_{0}, e_{1}, \psi, \psi^{\prime}\right\}^{\perp} \cap \operatorname{ker} Z \cap \operatorname{ker} Z^{*}$,

(ii)

$$
\rho=\frac{\Gamma_{R e,+, \omega_{2}}}{\Gamma_{R e,+, \omega_{2}}+\Gamma_{R e,-, \omega_{2}}}|u\rangle\left\langle u\left|+\frac{\Gamma_{R e,-, \omega_{2}}}{\Gamma_{R e,+, \omega_{2}}+\Gamma_{R e,-, \omega_{2}}} Z\right| u\right\rangle\langle u| Z^{*}
$$

con $u \in V \cap \operatorname{Im}|Z|$.

Demostración. Del teorema 3.1. cualquier estado de la forma $|u\rangle\langle u| \operatorname{con} u \in W_{D}$ es invariante y extremo. Recíprocamente, si $\rho$ es un estado invariante extremo soportado en $W_{D}$, entonces es necesariamente un estado puro, es decir, $\rho=|u\rangle\langle u|$ para algún $u \in W_{D}$.

Por otra parte, se verifica inmediatamente que cualquier estado $\rho$ de la forma (3.22) está soportado en $V \cap W_{D}^{\perp}$. Por consiguiente, del teorema 3.5, $\rho$ es invariante ya que tiene la estructura (3.20) con $\lambda=0$ y $\sigma=|u\rangle\langle u|$. Ahora, sea

$$
\rho=\lambda \rho_{1}+(1-\lambda) \rho_{2}
$$


con $\rho_{i}, i=1,2$ estados invariantes soportados en $V \cap W_{D}^{\perp}$, digamos

$$
\rho_{i}=\frac{\Gamma_{R e,+, \omega_{2}}}{\Gamma_{R e,+, \omega_{2}}+\Gamma_{R e,-, \omega_{2}}} \sigma_{i}+\frac{\Gamma_{R e,-, \omega_{2}}}{\Gamma_{R e,+, \omega_{2}}+\Gamma_{R e,-, \omega_{2}}} Z \sigma_{i} Z^{*}, \quad i=1,2 .
$$

con $\sigma_{i}=\left|u_{i}\right\rangle\left\langle u_{i}\right|$ soportado en $V \cap \operatorname{Im}|Z|, i=1,2$.

Posteriormente, multiplicando por $|Z|$ la ecuación (3.23) se obtiene

$$
|u\rangle\langle u|=\lambda \sigma_{1}+(1-\lambda) \sigma_{2} .
$$

Por lo tanto, $\lambda=1$ y $\sigma_{1}=|u\rangle\langle u|$ o $\lambda=0$ y $\sigma_{2}=|u\rangle\langle u|$, pues $|u\rangle\langle u|$ es un estado puro. Esto prueba que $\rho$ es extremo. Recíprocamente, si $\rho$ es un estado invariante extremo soportado en $V \cap W_{D}^{\perp}$, entonces tiene la estructura 3.20 $\operatorname{con} \lambda=0$. Por lo tanto, necesariamente

$$
\rho=\frac{\Gamma_{R e,+, \omega_{2}}}{\Gamma_{R e,+, \omega_{2}}+\Gamma_{R e,-, \omega_{2}}} \sigma+\frac{\Gamma_{R e,-, \omega_{2}}}{\Gamma_{R e,+, \omega_{2}}+\Gamma_{R e,-, \omega_{2}}} Z \sigma Z^{*}
$$

con $\sigma$ un estado soportado en $V \cap \operatorname{Im}|Z|$, digamos $\sigma=\sum_{j} \sigma_{j}\left|u_{j}\right\rangle\left\langle u_{j}\right| \operatorname{con}\left(u_{j}\right)_{j} \subset$ $V \cap \operatorname{Im}|Z|$ la base ortonormal de $\sigma \mathrm{y} \sum_{j} \sigma_{j}=1$. Consecuentemente,

$$
\rho=\sum_{j} \sigma_{j}\left(\frac{\Gamma_{R e,+, \omega_{2}}}{\Gamma_{R e,+, \omega_{2}}+\Gamma_{R e,-, \omega_{2}}}\left|u_{j}\right\rangle\left\langle u_{j}\left|+\frac{\Gamma_{R e,-, \omega_{2}}}{\Gamma_{R e,+, \omega_{2}}+\Gamma_{R e,-, \omega_{2}}}\right| Z u_{j}\right\rangle\left\langle Z u_{j}\right|\right)
$$

Pero $\rho$ es extremo, entonces sólo hay un sumando no trivial en (3.25). Esto termina la prueba.

Hasta ahora hemos encontrado y estudiado estados soportados en $W_{D} \oplus W_{D}^{\perp} \cap$ $V=\mathrm{h} \cap V=V$. La pregunta acerca de la existencia de estados soportados en $V^{\perp}=\left\{e_{0}, e_{1}, \psi, \psi^{\prime}, Z \psi, Z^{*} \psi^{\prime}\right\}$ surge de manera natural. Comenzaremos con el resultado siguiente.

Proposición 3.5. El estado $\left|e_{0}\right\rangle\left\langle e_{0}\right|$ es invariante para el modelo de transporte cuántico. Además, $\left|e_{0}\right\rangle\left\langle e_{0}\right|$ es extremo.

Demostración. Claramente, el soporte de $\left|e_{0}\right\rangle\left\langle e_{0}\right|$ es $\digamma_{0}:=\operatorname{span}\left\{e_{0}\right\}$. La estructura del Hamiltoniano efectivo (3.12) implica que $\left[H_{e f f},\left|e_{0}\right\rangle\left\langle e_{0}\right|\right]=0$. Por otra parte, la estructura de los operadores de Kraus implican que

$$
\begin{aligned}
& L_{k}^{\omega_{j *}} L_{k}^{\omega_{j}}\left|e_{0}\right\rangle\left\langle e_{0}\right|=0, \quad \forall 1 \leq k \leq 2, \quad \forall 1 \leq j \leq 3 . \\
& L_{k}^{\omega_{j}}\left|e_{0}\right\rangle\left\langle e_{0}\right| L_{k}^{\omega_{j} *}=0, \quad \forall 1 \leq k \leq 2, \quad \forall 1 \leq j \leq 3 . \\
& \left|e_{0}\right\rangle\left\langle e_{0}\right| L_{k}^{\omega_{j} *} L_{k}^{\omega_{j}}=0, \quad \forall 1 \leq k \leq 2, \quad \forall 1 \leq j \leq 3 .
\end{aligned}
$$


Consecuentemente, $\left|e_{0}\right\rangle\left\langle e_{0}\right|$ es invariante. Además, $\left|e_{0}\right\rangle\left\langle e_{0}\right|$ es un estado invariante puro. Por lo tanto, $\left|e_{0}\right\rangle\left\langle e_{0}\right|$ es un estado invariante extremo. Esto termina la prueba.

Proposición 3.6. No existen estados invariantes para el modelo de transporte cuántico soportados en $\digamma_{u}:=\operatorname{span}\{u\}$, para cada $u \in\left\{e_{1}, \psi, \psi^{\prime}, Z \psi, Z^{*} \psi^{\prime}\right\}$.

Demostración. Fijemos $u=e_{1}$ y sea $\rho=\rho_{11} P_{1}$ un estado soportado en $\digamma_{1}:=$ $\operatorname{span}\left\{e_{1}\right\}$. La estructura del Hamiltoniano efectivo 3.12 implica que $\left[H_{e f f}, \rho\right]=$ 0. Consecuentemente,

$$
\begin{aligned}
\mathcal{L}_{*}(\rho) & =2 \Gamma_{R e,-, \omega_{1}} \rho_{11}|\psi\rangle\langle\psi|-2 \Gamma_{R e,-, \omega_{1}} \rho_{11}\langle\psi, \psi\rangle P_{1} \\
& =2 \Gamma_{R e,-, \omega_{1}} \rho_{11}|\psi\rangle\langle\psi|-2(N-1) \Gamma_{R e,-, \omega_{1}} \rho_{11} P_{1}
\end{aligned}
$$

Dado que $|\psi\rangle\langle\psi|$ y $P_{1}$ tienen rangos mutuamente ortogonales y la positividad del coeficiente $\Gamma_{R e,-, \omega_{1}}$, implican que $\mathcal{L}_{*}(\rho)=0 \Longleftrightarrow \rho_{11}=0$. Consecuentemente, $\rho=0$, lo cual es imposible puesto que $\rho$ es un estado. Por lo tanto, no existen estados invariantes soportados en $\digamma_{1}:=\operatorname{span}\left\{e_{1}\right\}$. Ahora, fijemos $u=\psi$ y sea $\rho=\rho_{\psi}|\psi\rangle\langle\psi|$ un estado soportado en $\digamma_{\psi}:=\operatorname{span}\{\psi\}$. La estructura del Hamiltoniano efectivo 3.12 implica que $-i\left[H_{e f f}, \rho\right]=0$. En efecto,

$$
\begin{aligned}
-i\left[H_{e f f}, \rho\right] & =i(N-1) \Gamma_{I m,-, \omega_{2}} \rho_{\psi}|\psi\rangle\left\langle\psi\left|-i(N-1) \Gamma_{I m,+, \omega_{1}} \rho_{\psi}\right| \psi\right\rangle\langle\psi| \\
& +i(N-1) \Gamma_{I m,+, \omega_{1}} \rho_{\psi}|\psi\rangle\left\langle\psi\left|-i(N-1) \Gamma_{I m,-, \omega_{2}} \rho_{\psi}\right| \psi\right\rangle\langle\psi|=0 .
\end{aligned}
$$

Consecuentemente,

$$
\begin{aligned}
\mathcal{L}_{*}(\rho) & =2(N-1)^{2} \Gamma_{R e,+, \omega_{1}} \rho_{\psi}\left|e_{1}\right\rangle\left\langle e_{1}\left|-2(N-1) \Gamma_{R e,+, \omega_{1}} \rho_{\psi}\right| \psi\right\rangle\langle\psi| \\
& +2(N-1) \Gamma_{R e,-, \omega_{2}} \rho_{\psi}|Z \psi\rangle\left\langle Z \psi\left|-2(N-1) \Gamma_{R e,-, \omega_{2}} \rho_{\psi}\right| \psi\right\rangle\langle\psi| \\
& =2(N-1)^{2} \Gamma_{R e,+, \omega_{1}} \rho_{\psi}\left|e_{1}\right\rangle\left\langle e_{1}\left|+2(N-1) \Gamma_{R e,-, \omega_{2}} \rho_{\psi}\right| Z \psi\right\rangle\langle Z \psi| \\
& -2(N-1)\left(\Gamma_{R e,+, \omega_{1}}+\Gamma_{R e,-, \omega_{2}}\right) \rho_{\psi}|\psi\rangle\langle\psi|
\end{aligned}
$$

La positividad de los coeficientes $\Gamma$ 's y la ortogonalidad de los rangos implican que

$$
\mathcal{L}_{*}(\rho)=0 \Longleftrightarrow \rho_{\psi}=0 .
$$

Entonces, $\rho=0$, lo cual es imposible, puesto que $\rho$ es un estado, más aún, $\rho_{\psi}=$ $1 /(N-1)$. Consecuentemente, no hay estados invariantes soportados en $\digamma_{\psi}:=$ $\operatorname{span}\{\psi\}$. Análogamente, se verifica que no existen estados soportados en $\digamma_{\psi^{\prime}}:=$ $\operatorname{span}\left\{\psi^{\prime}\right\}, \digamma_{Z \psi}:=\operatorname{span}\{Z \psi\}$ y $\digamma_{Z^{*} \psi^{\prime}}:=\operatorname{span}\left\{Z^{*} \psi^{\prime}\right\}$. Esto termina la prueba. 
Proposición 3.7. No existen estados invariantes para el modelo de transporte cuántico soportados en $\digamma:=\operatorname{span}\left\{e_{1}, \psi, \psi^{\prime}, Z \psi, Z^{*} \psi^{\prime}\right\}$.

Demostración. Fijemos $u=a e_{1}+v \operatorname{con} v=b \psi+c \psi^{\prime}+d Z \psi+f Z^{*} \psi^{\prime}$ y $a, b, c, d, f \in \mathbb{C}$. Consideremos el estado $\rho_{u}=|u\rangle\left\langle\left. u|=| a\right|^{2} \mid e_{1}\right\rangle\left\langle e_{1}|+a| e_{1}\right\rangle\langle v|+$ $\bar{a}|v\rangle\left\langle e_{1}|+| v\right\rangle\langle v|$. Cálculos directos verifican que $\mathcal{L}_{*}\left(\rho_{u}\right)=0$ si y sólo si

i) $\left\langle\psi^{\prime}, v\right\rangle=0$.

ii) $\Gamma_{R e,+, \omega_{1}}|\langle\psi, v\rangle|^{2}=|a|^{2}(N-1) \Gamma_{R e,-, \omega_{1}}$.

iii) $\mathcal{L}_{*}^{R}\left(\rho_{u}\right)=0$, donde

$$
\begin{aligned}
\mathcal{L}_{*}^{R}\left(\rho_{u}\right) & =-a i\left[-(N-1) \Gamma_{I m,-, \omega_{1}}\left|e_{1}\right\rangle\left\langle v\left|-\Gamma_{I m,+, \omega_{1}}\langle v, \psi\rangle\right| e_{1}\right\rangle\langle\psi|\right. \\
& -(N-1) \Gamma_{I m,+, \omega_{2}}\left|e_{1}\right\rangle\left\langle P_{3} v\left|+(N-1) \Gamma_{I m,-, \omega_{2}}\right| e_{1}\right\rangle\langle Z|v| \\
& \left.+\Gamma_{I m,-, \omega_{3}}\left\langle v, \psi^{\prime}\right\rangle\left|e_{1}\right\rangle\left\langle\psi^{\prime}\right|\right] \\
& -\frac{a}{2}\left[2(N-1) \Gamma_{R e,-, \omega_{1}}\left|e_{1}\right\rangle\left\langle v\left|+2 \Gamma_{R e,+, \omega_{1}}\langle v, \psi\rangle\right| e_{1}\right\rangle\langle\psi|\right. \\
& +2(N-1) \Gamma_{R e,-, \omega_{2}}\left|e_{1}\right\rangle\left\langle Z|v|+2(N-1) \Gamma_{R e,+, \omega_{2}} \mid e_{1}\right\rangle\left\langle P_{3} v\right| \\
& \left.+2 \Gamma_{R e,-, \omega_{3}}\left\langle v, \psi^{\prime}\right\rangle\left|e_{1}\right\rangle\left\langle\psi^{\prime}\right|\right] \\
& -\bar{a}\left[\left[\Gamma_{I m,+, \omega_{1}}\langle\psi, v\rangle|\psi\rangle\left\langle e_{1}\left|+(N-1) \Gamma_{I m,+, \omega_{2}}\right| P_{3} v\right\rangle\left\langle e_{1}\right|\right.\right. \\
& -(N-1) \Gamma_{I m,-, \omega_{2}}|| Z|v\rangle\left\langle e_{1}\left|+(N-1) \Gamma_{I m,-, \omega_{1}}\right| v\right\rangle\left\langle e_{1}\right| \\
& \left.-\Gamma_{I m,-, \omega_{3}}\left\langle\psi^{\prime}, v\right\rangle\left|\psi^{\prime}\right\rangle\left\langle e_{1}\right|\right] \\
& -\frac{\bar{a}}{2}\left[2(N-1) \Gamma_{R e,-, \omega_{1}}|v\rangle\left\langle e_{1}\left|+2 \Gamma_{R e,+, \omega_{1}}\langle\psi, v\rangle\right| \psi\right\rangle\left\langle e_{1}\right|\right. \\
& +2(N-1) \Gamma_{R e,-, \omega_{2}}|| Z|v\rangle\left\langle e_{1}\left|+2(N-1) \Gamma_{R e,+, \omega_{2}}\right| P_{3} v\right\rangle\left\langle e_{1}\right| \\
& \left.+2 \Gamma_{R e,-, \omega_{3}}\left\langle\psi^{\prime}, v\right\rangle\left|\psi^{\prime}\right\rangle\left\langle e_{1}\right|\right] \\
& -i\left[\Gamma_{I m,+, \omega_{1}}\langle\psi, v\rangle|\psi\rangle\left\langle v\left|+(N-1) \Gamma_{I m,+, \omega_{2}}\right| P_{3} v\right\rangle\langle v|\right. \\
& -(N-1) \Gamma_{I m,-, \omega_{2}}|| Z|v\rangle\left\langle v\left|-\Gamma_{I m,-, \omega_{3}}\left\langle\psi^{\prime}, v\right\rangle\right| \psi^{\prime}\right\rangle\langle v| \\
& -\Gamma_{I m,+, \omega_{1}}\langle v, \psi\rangle|v\rangle\left\langle\psi\left|-(N-1) \Gamma_{I m,+, \omega_{2}}\right| v\right\rangle\left\langle P_{3} v\right| \\
& \left.+(N-1) \Gamma_{I m,-, \omega_{2}}|v\rangle\left\langle Z|v|+\Gamma_{I m,-, \omega_{3}}\left\langle v, \psi^{\prime}\right\rangle \mid v\right\rangle\left\langle\psi^{\prime}\right|\right] \\
& -\frac{1}{2}\left[2 \Gamma_{R e,+, \omega_{1}}\langle\psi, v\rangle|\psi\rangle\left\langle v\left|+2 \Gamma_{R e,+, \omega_{1}}\langle v, \psi\rangle\right| v\right\rangle\langle\psi|\right. \\
& +2(N-1) \Gamma_{R e,-, \omega_{2}}|| Z|v\rangle\left\langle v\left|-4(N-1) \Gamma_{R e,-, \omega_{2}}\right| Z v\right\rangle\langle Z v| \\
& +2(N-1) \Gamma_{R e,-, \omega_{2}}|v\rangle\left\langle|Z| v\left|+2(N-1) \Gamma_{R e,+, \omega_{2}}\right| P_{3} v\right\rangle\langle v| \\
& -4(N-1) \Gamma_{R e,+, \omega^{\prime}}\left|Z^{*} v\right\rangle\left\langle Z^{*} v\left|+2(N-1) \Gamma_{R e,+, \omega_{2}}\right| v\right\rangle\left\langle P_{3} v\right| \\
& \left.+2 \Gamma_{R e,-, \omega_{3}}\left(\left\langle\psi^{\prime}, v\right\rangle\left|\psi^{\prime}\right\rangle\left\langle v\left|+\left\langle v, \psi^{\prime}\right\rangle\right| v\right\rangle\left\langle\psi^{\prime}\right|\right)-4|a|^{2} \Gamma_{R e,-, \omega_{1}}|\psi\rangle\langle\psi|\right] . \\
&
\end{aligned}
$$


Supongamos $a \neq 0$. Obsérvese que $\mathcal{L}_{*}^{R}\left(\rho_{u}\right)=\left(P_{1}+P_{2}+P_{3}\right) \mathcal{L}_{*}^{R}\left(\rho_{u}\right)\left(P_{1}+P_{2}+\right.$ $\left.P_{3}\right)=P_{1} \mathcal{L}_{*}^{R}\left(\rho_{u}\right) P_{1}+P_{1} \mathcal{L}_{*}^{R}\left(\rho_{u}\right) P_{2}+P_{1} \mathcal{L}_{*}^{R}\left(\rho_{u}\right) P_{3}+P_{2} \mathcal{L}_{*}^{R}\left(\rho_{u}\right) P_{1}+P_{2} \mathcal{L}_{*}^{R}\left(\rho_{u}\right) P_{2}+$ $P_{2} \mathcal{L}_{*}^{R}\left(\rho_{u}\right) P_{3}+P_{3} \mathcal{L}_{*}^{R}\left(\rho_{u}\right) P_{1}+P_{3} \mathcal{L}_{*}^{R}\left(\rho_{u}\right) P_{2}+P_{3} \mathcal{L}_{*}\left(\rho_{u}\right) P_{3}$. Por lo tanto, $\mathcal{L}_{*}^{R}\left(\rho_{u}\right)=$ 0 si y sólo si $P_{l} \mathcal{L}_{*}^{R}\left(\rho_{u}\right) P_{k}=0, \forall 1 \leq l, k \leq 3$. Mediante cálculos directos se obtiene que

$$
P_{1} \mathcal{L}_{*}^{R}\left(\rho_{u}\right) P_{3}=a(N-1)\left[i\left(\Gamma_{I m,-, \omega_{1}}+\Gamma_{I m,+, \omega_{1}}\right)-\left(\Gamma_{R e,-, \omega_{1}}+\Gamma_{R e,+, \omega_{1}}\right)\right]\left|e_{1}\right\rangle\left\langle P_{3} v\right| .
$$

Consecuentemente, $P_{1} \mathcal{L}_{*}^{R}\left(\rho_{u}\right) P_{3}=0$ si y sólo si $P_{3} v=0$. Entonces, $P_{3} v=c \psi^{\prime}+$ $d Z \psi=0$, además, $\psi^{\prime}$ y $Z \psi$ son linealmente independientes, entonces $c=d=0$. Consecuentemente, $v=b \psi+f Z^{*} \psi^{\prime}$.

Por otra parte, recordemos que $Z^{*} \psi^{\prime}=\sqrt{N-1} e_{N-1}$ y reescribiendo a $\psi=$ $\xi+\left|e_{N-1}\right\rangle$, donde $\xi=\sum_{k=2, k \neq N-1}^{N}\left|e_{k}\right\rangle$, entonces $v=b \psi+f Z^{*} \psi^{\prime}=b \xi+(b+$ $f \sqrt{N-1})\left|e_{N-1}\right\rangle$. Consecuentemente,

$$
\begin{aligned}
P_{1} \mathcal{L}_{*}^{R}\left(\rho_{u}\right) P_{2} & =a\left[i(N-1)\left(\Gamma_{I m,-, \omega_{1}}-\Gamma_{I m,-, \omega_{2}}\right) \bar{b}+i \Gamma_{I m,+, \omega_{1}}\langle v, \psi\rangle\right. \\
& \left.-(N-1)\left(\Gamma_{R e,-, \omega_{1}}+\Gamma_{R e,-, \omega_{2}}\right) \bar{b}-\Gamma_{R e,+, \omega_{1}}\langle v, \psi\rangle\right]\left|e_{1}\right\rangle\langle\xi| \\
& +a\left[i(N-1)\left(\Gamma_{I m,-, \omega_{1}}-\Gamma_{I m,-, \omega_{2}}\right)(\bar{b}+\bar{f} \sqrt{N-1})+i \Gamma_{I m,+, \omega_{1}}\langle v, \psi\rangle\right. \\
& \left.-(N-1)\left(\Gamma_{R e,-, \omega_{1}}+\Gamma_{R e,-, \omega_{2}}\right)(\bar{b}+\bar{f} \sqrt{N-1})-\Gamma_{R e,+, \omega_{1}}\langle v, \psi\rangle\right]\left|e_{1}\right\rangle\left\langle e_{N-1}\right| .
\end{aligned}
$$

Dado que $\xi$ y $\left|e_{N-1}\right\rangle$ son ortogonales, se verifica que $P_{1} \mathcal{L}_{*}^{R}\left(\rho_{u}\right) P_{2}=0$ si y sólo si

i) $i(N-1)\left(\Gamma_{I m,-, \omega_{1}}-\Gamma_{I m,-, \omega_{2}}\right) \bar{b}+i \Gamma_{I m,+, \omega_{1}}\langle v, \psi\rangle-(N-1)\left(\Gamma_{R e,-, \omega_{1}}+\right.$ $\left.\Gamma_{R e,-, \omega_{2}}\right) \bar{b}-\Gamma_{R e,+, \omega_{1}}\langle v, \psi\rangle=0$.

ii) $i(N-1)\left(\Gamma_{I m,-, \omega_{1}}-\Gamma_{I m,-, \omega_{2}}\right)(\bar{b}+\bar{f} \sqrt{N-1})+i \Gamma_{I m,+, \omega_{1}}\langle v, \psi\rangle-(N-$ 1) $\left(\Gamma_{R e,-, \omega_{1}}+\Gamma_{R e,-, \omega_{2}}\right)(\bar{b}+\bar{f} \sqrt{N-1})-\Gamma_{R e,+, \omega_{1}}\langle v, \psi\rangle=0$.

Entonces, mediante cálculos directos se verifica que

$$
(N-1)\left[i\left(\Gamma_{I m,-, \omega_{1}}-\Gamma_{I m,-, \omega_{2}}\right)-\left(\Gamma_{R e,-, \omega_{1}}+\Gamma_{R e,-, \omega_{2}}\right)\right] \bar{f} \sqrt{N-1}=0 .
$$

La positividad de los coeficientes $\Gamma$ 's, implica que $f=0$, Consecuentemente, $v=b \psi$. Finalmente, dado que $Z \psi=\sqrt{N-1} e_{N+1}$ y $|Z| \psi=P_{2} \psi=\psi$, entonces

$$
\begin{aligned}
P_{2} \mathcal{L}_{*}^{R}\left(\rho_{u}\right) P_{2} & =\left[2|a|^{2} \Gamma_{R e,-, \omega_{1}}-2(N-1)|b|^{2}\left(\Gamma_{R e,+, \omega_{1}}+\Gamma_{R e,-, \omega_{2}}\right)\right]|\psi\rangle\langle\psi| \\
& +2(N-1)^{2}|b|^{2} \Gamma_{R e,-, \omega_{2}}\left|e_{N+1}\right\rangle\left\langle e_{N+1}\right| .
\end{aligned}
$$


Por lo tanto, la positividad de los coeficientes $\Gamma$ 's y la ortogonalidad de los rangos de $|\psi\rangle\langle\psi|$ y $\left|e_{N+1}\right\rangle\left\langle e_{N+1}\right|$ implican que $P_{2} \mathcal{L}_{*}^{R}\left(\rho_{u}\right) P_{2}=0$ si y sólo si $b=0$ y $a=0$. Lo cual es una contradicción. Por lo tanto, $a=0$. Fijemos $v=b \psi+c \psi^{\prime}+$ $d Z \psi+f Z^{*} \psi^{\prime} \operatorname{con} b, c, d, f \in \mathbb{C}$. Consideremos el estado $\rho_{v}=|v\rangle\langle v|$. Entonces,

$$
\begin{aligned}
\mathcal{L}_{*}\left(\rho_{v}\right) & =2 \Gamma_{R e,-, \omega_{3}}\left|\left\langle\psi^{\prime}, v\right\rangle\right|^{2} P_{0} \\
& +2 \Gamma_{R e,+, \omega_{1}}|\langle\psi, v\rangle|^{2} P_{1} \\
& -i\left[\Gamma_{I m,+, \omega_{1}}\langle\psi, v\rangle|\psi\rangle\left\langle v\left|+(N-1) \Gamma_{I m,+, \omega_{2}}\right| P_{3} v\right\rangle\langle v|\right. \\
& -(N-1) \Gamma_{I m,-, \omega_{2}}|| Z|v\rangle\left\langle v\left|-\Gamma_{I m,-, \omega_{3}}\left\langle\psi^{\prime}, v\right\rangle\right| \psi^{\prime}\right\rangle\langle v| \\
& -\Gamma_{I m,+, \omega_{1}}\langle v, \psi\rangle|v\rangle\left\langle\psi\left|-(N-1) \Gamma_{I m,+, \omega_{2}}\right| v\right\rangle\left\langle P_{3} v\right| \\
& \left.+(N-1) \Gamma_{I m,-, \omega_{2}}|v\rangle\left\langle|Z| v\left|+\Gamma_{I m,-, \omega_{3}}\left\langle v, \psi^{\prime}\right\rangle\right| v\right\rangle\left\langle\psi^{\prime}\right|\right] \\
& -\frac{1}{2}\left[2 \Gamma_{R e,+, \omega_{1}}\langle\psi, v\rangle|\psi\rangle\left\langle v\left|+2 \Gamma_{R e,+, \omega_{1}}\langle v, \psi\rangle\right| v\right\rangle\langle\psi|\right. \\
& +2(N-1) \Gamma_{R e,-, \omega_{2}}|| Z|v\rangle\left\langle v\left|-4(N-1) \Gamma_{R e,-, \omega_{2}}\right| Z v\right\rangle\langle Z v| \\
& +2(N-1) \Gamma_{R e,-, \omega_{2}}|v\rangle\left\langle|Z| v\left|+2(N-1) \Gamma_{R e,+, \omega_{2}}\right| P_{3} v\right\rangle\langle v| \\
& -4(N-1) \Gamma_{R e,+, \omega_{2}}\left|Z^{*} v\right\rangle\left\langle Z^{*} v\left|+2(N-1) \Gamma_{R e,+, \omega_{2}}\right| v\right\rangle\left\langle P_{3} v\right| \\
& \left.+2 \Gamma_{R e,-, \omega_{3}}\left\langle\psi^{\prime}, v\right\rangle\left|\psi^{\prime}\right\rangle\left\langle\left. v|-4| a\right|^{2} \Gamma_{R e,-, \omega_{1}} \mid \psi\right\rangle\left\langle\psi\left|+2 \Gamma_{R e,-, \omega_{3}}\left\langle v, \psi^{\prime}\right\rangle\right| v\right\rangle\left\langle\psi^{\prime}\right|\right] .
\end{aligned}
$$

Consecuentemente, $\mathcal{L}_{*}\left(\rho_{v}\right)=0$ si y sólo si

i) $\langle\psi, v\rangle=0$.

ii) $\left\langle\psi^{\prime}, v\right\rangle=0$.

iii) $\mathcal{L}_{*}^{R}\left(\rho_{v}\right)=0$, donde

$$
\begin{aligned}
\mathcal{L}_{*}^{R}\left(\rho_{v}\right) & =-i\left[(N-1) \Gamma_{I m,+, \omega_{2}}\left|P_{3} v\right\rangle\left\langle v\left|-(N-1) \Gamma_{I m,-, \omega_{2}}\right||Z| v\right\rangle\langle v|\right. \\
& \left.-(N-1) \Gamma_{I m,+, \omega_{2}}|v\rangle\left\langle P_{3} v\left|+(N-1) \Gamma_{I m,-, \omega_{2}}\right| v\right\rangle\langle|Z| v|\right] \\
& -\frac{1}{2}\left[2(N-1) \Gamma_{R e,-, \omega_{2}}|| Z|v\rangle\left\langle v\left|-4(N-1) \Gamma_{R e,-, \omega_{2}}\right| Z v\right\rangle\langle Z v|\right. \\
& +2(N-1) \Gamma_{R e,-, \omega_{2}}|v\rangle\left\langle|Z| v\left|+2(N-1) \Gamma_{R e,+, \omega_{2}}\right| P_{3} v\right\rangle\langle v| \\
& \left.-4(N-1) \Gamma_{R e,+, \omega_{2}}\left|Z^{*} v\right\rangle\left\langle Z^{*} v\left|+2(N-1) \Gamma_{R e,+, \omega_{2}}\right| v\right\rangle\left\langle P_{3} v\right|\right] .
\end{aligned}
$$


Por otra parte, $\mathcal{L}_{*}^{R}\left(\rho_{v}\right)=\left(P_{2}+P_{3}\right) \mathcal{L}_{*}^{R}\left(\rho_{v}\right)\left(P_{2}+P_{3}\right)=P_{2} \mathcal{L}_{*}^{R}\left(\rho_{v}\right) P_{2}+P_{2} \mathcal{L}_{*}^{R}\left(\rho_{v}\right) P_{3}$ $+P_{3} \mathcal{L}_{*}^{R}\left(\rho_{v}\right) P_{2}+P_{3} \mathcal{L}_{*}^{R}\left(\rho_{v}\right) P_{3}$. De los rangos mutuamente ortogonales, $\mathcal{L}_{*}^{R}\left(\rho_{v}\right)=$ 0 si y sólo si cada bloque $P_{l} \mathcal{L}_{*}\left(\rho_{v}\right) P_{k}=0, \forall 2 \leq l, k \leq 3$. Entonces,

$$
\begin{aligned}
& P_{2} \mathcal{L}_{*}\left(\rho_{v}\right) P_{2}=2(N-1)\left[\Gamma_{R e,+, \omega_{2}}\left|P_{2} Z^{*} v\right\rangle\left\langle P_{2} Z^{*} v\left|-\Gamma_{R e,-, \omega_{2}}\right||Z| v\right\rangle\langle|Z| v|\right] \\
& P_{3} \mathcal{L}_{*}\left(\rho_{v}\right) P_{3}=2(N-1)\left[\Gamma_{R e,-, \omega_{2}}|Z v\rangle\left\langle Z v\left|-\Gamma_{R e,+, \omega_{2}}\right| P_{3} v\right\rangle\left\langle P_{3} v\right|\right] \\
& P_{2} \mathcal{L}_{*}\left(\rho_{v}\right) P_{3}=(N-1)\left[i\left(\Gamma_{I m,-, \omega_{2}}+\Gamma_{I m,+, \omega_{2}}\right)-\left(\Gamma_{R e,-, \omega_{2}}+\Gamma_{R e,+, \omega_{2}}\right)\right]\left|P_{2} v\right\rangle\left\langle P_{3} v\right| \\
& P_{3} \mathcal{L}_{*}\left(\rho_{v}\right) P_{2}=(N-1)\left[-i\left(\Gamma_{I m,-, \omega_{2}}+\Gamma_{I m,+, \omega_{2}}\right)-\left(\Gamma_{R e,-, \omega_{2}}+\Gamma_{R e,+, \omega_{2}}\right)\right]\left|P_{3} v\right\rangle\left\langle P_{2} v\right| .
\end{aligned}
$$

La positividad de los coeficientes $\Gamma$ 's y las últimas dos ecuaciones anteriores implican que $P_{2} v=0$ o $P_{3} v=0$. Si $P_{3} v=0$, entonces de la segunda ecuación en (3.26) se obtiene que $Z v=0$. Consecuentemente, $|Z| v=Z^{*} Z v=0$. Además, $P_{2} v=|Z| v$, la primer ecuación en (3.26) implica que $P_{2} Z^{*} v=Z^{*} v=0$. Por lo tanto, $v=\left(P_{2}+P_{3}\right) v=P_{2} v+P_{3} v=|Z| v+P_{3} v=0$. Análogamente, si $P_{2} v=0$ y dado que $|Z| v=P_{2} v=0$, la primer ecuación en 3.26 implica que $P_{2} Z^{*} v=Z^{*} v=0$, consecuentemente, $P_{3} v=Z Z^{*} v=0$, la segunda ecuación en (3.26) implica que $Z v=0$. Por lo tanto, $v=\left(P_{2}+P_{3}\right) v=P_{2} v+P_{3} v=$ $|Z| v+P_{3} v=0$. Consecuentemente, no hay estados invariantes soportados en $\digamma:=\operatorname{span}\left\{e_{1}, \psi, \psi^{\prime}, Z \psi, Z^{*} \psi^{\prime}\right\}$. Esto termina la prueba.

Corolario 3.1. Todos los estados invariantes conocidos para el modelo de transporte cuántico son $\left|e_{0}\right\rangle\left\langle e_{0}\right|$ o tienen la estructura (3.21).

Corolario 3.2. Todos los estados invariantes conocidos del modelo de transporte cuántico son de balance detallado (de acuerdo a la definición 3.2).

Demostración. Si $\rho=\left|e_{0}\right\rangle\left\langle e_{0}\right|$ el resultado se sigue inmediatamente. Sea $\rho$ un estado invariante de la forma (3.21). Claramente la parte de $\rho$ soportada en $W_{D}$ satisface una condición de balance detallado, por lo que se puede suponer que $\rho$ está soportado en $V \cap W_{D}^{\perp}$. Por otra parte, recordemos que $\left\{e_{0}, e_{1}, \psi, \psi^{\prime}\right\} \subset \operatorname{ker} \rho$ si $\rho$ es un estado invariante soportado en $V \cap W_{D}^{\perp}$ y $|Z| \psi=\psi$. Mediante cálculos directos se verifica que $\rho D_{\omega_{1}}=0=D_{\omega_{1}} \rho$ y $\rho D_{\omega_{3}}=0=D_{\omega_{3}} \rho$. Además,

$$
\rho D_{\omega_{2}}=\frac{\Gamma_{R e,-, \omega_{2}}}{\Gamma_{R e,+, \omega_{2}}} Z \rho
$$

$\mathrm{y}$

$$
D_{\omega_{2}} \rho=Z \rho .
$$

Por lo tanto, $\rho D_{\omega_{2}}=\frac{\Gamma_{R e,-, \omega_{2}}}{\Gamma_{R e,+, \omega_{2}}} D_{\omega_{2}} \rho$. Como consecuencia de la definición 3.2, se concluye que $\rho$ es un estado de balance detallado. 


\subsubsection{Subespacio de recurrencia rápida}

El subespacio de recurrencia rápida de un semigrupo cuántico de Markov se define como

$$
\mathcal{R}:=\sup \{s(\rho): s(\rho) \text { es el soporte de un estado invariante }\}
$$

En esta sección se caracteriza el subespacio de recurrencia rápida del modelo de transporte cuántico de [28].

Lema 3.3. Con las mismas notaciones de antes, se verifica que:

(i) $P_{W_{D}}=P_{2}-|Z|$.

(ii) $P_{W_{D}^{\perp}}=P_{0}+P_{1}+|Z|+P_{3}$.

(iii) $W_{D} \subset V$.

(iv) $V \cap W_{D}^{\perp}=\operatorname{Im}|Z| \cap\left\{\psi, Z^{*} \psi^{\prime}\right\}^{\perp} \oplus \operatorname{Im} P_{3} \cap\left\{\psi^{\prime}, Z \psi\right\}^{\perp}$.

Demostración. Para probar $(i)$ basta probar la identidad $W_{D}=\left\{\left(P_{2}-|Z|\right) v: v \in\right.$ h\}. En efecto, si $u=\left(P_{2}-|Z|\right) v$ con $v \in \mathrm{h}$ claramente $u \perp\left\{e_{0}, e_{1}\right\}$ y $u \perp \psi^{\prime}$, dado que $\psi^{\prime} \in \operatorname{ImP}_{3}$. Cálculos simples verifican que

$$
\langle\psi, u\rangle=\left\langle\psi,\left(P_{2}-|Z|\right) v\right\rangle=\left\langle\left(P_{2}-|Z|\right) \psi, u\right\rangle=0,
$$

puesto que $P_{2} \psi=\psi=|Z| \psi$, consecuentemente $u \perp \psi$. Además, $|Z| u=$ $|Z|\left(P_{2}-|Z|\right) v=0$ implica que $u \in \operatorname{ker}|Z|$ y claramente $Z^{*} u=0$. Esto prueba que $\left\{\left(P_{2}-|Z|\right) v: v \in \mathrm{h}\right\} \subset W_{D}$. Para probar la otra inclusión, observe que cualquier $u \in \mathrm{h}$ puede escribirse en la forma $u=P_{0} u+P_{1} u+P_{2} u+P_{3} u$, pero además, si $u \in W_{D}$, entonces $P_{0} u=0$ y $P_{1} u=0$. La condición $u \in \operatorname{ker}|Z|$ y la igualdad $P_{2}=\operatorname{Im}|Z| \oplus \operatorname{ker}|Z|$, implican que $u=\left(P_{2}-|Z|\right) v$ para algún $v \in \mathrm{h}$. Por lo tanto, $P_{3} u=0$. Esto prueba $(i)$.

(ii) es consecuencia inmediata de $(i)$. Ahora, para demostrar $($ iii $)$, sea $u \in W_{D}$, digamos $u=\left(P_{2}-|Z|\right) v, v \in \mathrm{h}$, entonces claramente $u \perp\left\{e_{0}, e_{1}\right\}$ y usando que $P_{2} \psi=\psi=|Z| \psi$, se obtiene que

$$
\langle\psi, u\rangle=\left\langle\psi,\left(P_{2}-|Z|\right) v\right\rangle=\left\langle\left(P_{2}-|Z|\right) \psi, u\right\rangle=0,
$$

es decir, $u \perp \psi$. Análogamente, las identidades $P_{2} Z^{*} \psi^{\prime}=Z^{*} \psi^{\prime}=|Z| Z^{*} \psi^{\prime}$ implican que $\left\langle Z^{*} \psi^{\prime}, u\right\rangle=\left\langle Z^{*} \psi^{\prime},\left(P_{2}-|Z|\right) v\right\rangle=\left\langle\left(P_{2}-|Z|\right) Z^{*} \psi^{\prime}, u\right\rangle=0$, es 
decir, $u \perp Z^{*} \psi^{\prime}$. Además, $u \in\left\{\psi^{\prime}, Z \psi\right\}^{\perp}$, pues $\psi^{\prime}, Z \psi \in \operatorname{Im} P_{3}$. Esto prueba que $W_{D} \subset V$.

Finalmente, usando (ii) se obtiene que

$$
\begin{aligned}
V \cap W_{D}^{\perp} & =\left\{e_{0}, e_{1}, \psi, \psi^{\prime}, Z \psi, Z^{*} \psi^{\prime}\right\}^{\perp} \cap\left(P_{1}+|Z|+P_{3}\right)(\mathrm{h}) \\
& =\left\{\psi, \psi^{\prime}, Z \psi, Z^{*} \psi^{\prime}\right\}^{\perp} \cap\left(|Z|+P_{3}\right)(\mathrm{h}) \\
& =I m|Z| \cap\left\{\psi, Z^{*} \psi^{\prime}\right\}^{\perp} \oplus \operatorname{Im} P_{3} \cap\left\{\psi^{\prime}, Z \psi\right\}^{\perp} .
\end{aligned}
$$

Esto prueba $(i v)$ y termina la prueba del lema.

Observación 3.14. Si $M=N-1$, equivalentemente $P_{2}=|Z|$, entonces $P_{W_{D}}=0$ y $P_{W_{D}^{\perp}}=\mathbb{1}$ (equivalentemente, $W_{D}=\{0\}$ y $W_{D}^{\perp}=\mathrm{h}$ ). Consecuentemente, todos los estados invariantes del modelo de transporte cuántico pertenecen a $\{H\}^{\prime}$.

Corolario 3.3. Si $M<N-1$, existen estados invariantes en el modelo de transporte cuántico que pertenecen a $A n n(D) \backslash\{H\}^{\prime}$.

Demostración. Sea $u=\left(P_{2}-|Z|\right) v \in W_{D} \operatorname{con} v \in \mathrm{h}$. Entonces,

$$
\begin{aligned}
P_{2}|u\rangle\langle u| P_{2} & =|u\rangle\langle u| \\
& =\left|\left(P_{2}-|Z|\right) v\right\rangle\left\langle\left(P_{2}-|Z|\right) v\right| \\
& =\left|P_{2} v-\right| Z|v\rangle\left\langle P_{2} v-|Z| v\right| \\
& =\left|P_{2} v-\right| Z|v\rangle\left\langle P_{2} v|-| P_{2} v-|Z| v\right\rangle\langle|Z| v| \\
& =\left|P_{2} v\right\rangle\left\langle P_{2} v|-||Z| v\right\rangle\left\langle P_{2} v|-| P_{2} v\right\rangle\langle|Z| v|+||Z| v\rangle\langle|Z| v|
\end{aligned}
$$

Por lo tanto, el estado invariante $|u\rangle\langle u|$ no pertenece a $\{H\}^{\prime}$ si $v \neq 0$ y $P_{2} v \neq|Z| v$, es decir, $|Z|<P_{2}$ (equivalentemente $M<N-1$, ver proposición 2.2 en [28]). No obstante, del teorema 3.2 y corolario 3.2, se sigue que todos los estados invariantes del modelo de transporte cuántico pertenecen a $\operatorname{Ann}(D)$.

Observación 3.15. Note que en contraposición con la referencia [28], no se asume que cualquier estado invariante $\tau$ pertenece a $\{H\}^{\prime}$. De hecho, existen estados invariantes $\tau$ fuera de $\{H\}^{\prime}$ cuando $|Z|<P_{2}$ (equivalentemente, $M<N-1$ ), pero pertenecen al Ann $(D)$ como se mostró previamente.

\section{Proposición 3.8.}

(i) Las restricciones de los operadores (sobre los dominios correspondientes) $Z: \operatorname{Im}|Z| \cap\left\{\psi, Z^{*} \psi^{\prime}\right\}^{\perp} \mapsto \operatorname{Im} P_{3} \cap\left\{\psi^{\prime}, Z \psi\right\}^{\perp} y Z^{*}: \operatorname{Im} P_{3} \cap\left\{\psi^{\prime}, Z \psi\right\}^{\perp} \mapsto$ $\operatorname{Im}|Z| \cap\left\{\psi, Z^{*} \psi^{\prime}\right\}^{\perp}$ son isometrías. Consecuentemente,

$$
\operatorname{dim}\left(\operatorname{Im}|Z| \cap\left\{\psi, Z^{*} \psi^{\prime}\right\}^{\perp}\right)=\operatorname{dim}\left(\operatorname{Im} P_{3} \cap\left\{\psi^{\prime}, Z \psi\right\}^{\perp}\right) .
$$


(ii) El estado

$$
\rho=\frac{1}{\operatorname{tr}(q)}\left(\frac{\Gamma_{R e,+, \omega_{2}}}{\Gamma_{R e,+, \omega_{2}}+\Gamma_{R e,-, \omega_{2}}} q+\frac{\Gamma_{R e,-, \omega_{2}}}{\Gamma_{R e,+, \omega_{2}}+\Gamma_{R e,-, \omega_{2}}} Z q Z^{*}\right)
$$

es invariante para el modelo de transporte cuántico, donde $q=|Z| P_{\left\{\psi, Z^{*} \psi^{\prime}\right\}^{\perp}}$ es la proyección ortogonal sobre $V \cap \operatorname{Im}|Z|$, con proyección soporte $P_{V \cap W_{D}^{\perp}}$ $=q+Z q Z^{*}$. Por lo tanto, $\rho$ es un estado fiel en la subálgebra hereditaria $P_{W_{D}^{\perp} \cap V} B(\mathrm{~h}) P_{W_{D}^{\perp} \cap V}$.

Demostración. Nótese que $Z$ envía $\operatorname{Im}|Z| \cap\left\{\psi, Z^{*} \psi^{\prime}\right\}^{\perp}$ en $\operatorname{Im} P_{3} \cap\left\{\psi^{\prime}, Z \psi\right\}^{\perp}$. En efecto, sea $u \in \operatorname{Im}|Z| \cap\left\{\psi, Z^{*} \psi^{\prime}\right\}^{\perp}$ entonces $P_{3} Z u=Z u,\left\langle\psi^{\prime}, Z u\right\rangle=\left\langle Z^{*} \psi^{\prime}, u\right\rangle$ $=0 \mathrm{y}\langle Z \psi, Z u\rangle=\langle|Z| \psi, u\rangle=\langle\psi, u\rangle=0$. Por lo tanto, $Z u \in \operatorname{ImP}_{3} \cap\left\{\psi^{\prime}, Z \psi\right\}^{\perp}$. Además, $\langle Z u, Z v\rangle=\langle|Z| u, v\rangle=\langle u, v\rangle$, significando que $Z$ es una isometría. Análogamente se prueba que $Z^{*}$ envía $\operatorname{Im} P_{3} \cap\left\{\psi^{\prime}, Z \psi\right\}^{\perp}$ en $\operatorname{Im}|Z| \cap\left\{\psi, Z^{*} \psi^{\prime}\right\}^{\perp}$. En efecto, sea $v \in \operatorname{Im} P_{3} \cap\left\{\psi^{\prime}, Z \psi\right\}^{\perp}$ entonces $|Z| Z^{*} v=Z^{*} v,\left\langle\psi, Z^{*} v\right\rangle=$ $\langle Z \psi, v\rangle=0$ y $\left\langle Z^{*} \psi^{\prime}, Z^{*} v\right\rangle=\left\langle P_{3} \psi^{\prime}, v\right\rangle=\left\langle\psi^{\prime}, v\right\rangle=0$. Por lo tanto, $Z^{*} v \in$ $\operatorname{Im}|Z| \cap\left\{\psi, Z^{*} \psi^{\prime}\right\}^{\perp}$. Además, $\left\langle Z^{*}, Z^{*} v\right\rangle=\left\langle u, P_{3} v\right\rangle=\langle u, v\rangle$, es decir, $Z^{*}$ es una isometría. Esto prueba $(i)$.

Sea $q$ la proyección ortogonal sobre el subespacio $V \cap \operatorname{Im}|Z|$. Observe que $q=|Z|$ sobre $\operatorname{Im}|Z| \cap\left\{\psi, Z^{*} \psi^{\prime}\right\}^{\perp}$. De hecho, dado que $\psi$ y $Z^{*} \psi^{\prime}$ son puntos fijos de $|Z|$, este operador $|Z|$ envía $\operatorname{Im}|Z| \cap\left\{\psi, Z^{*} \psi^{\prime}\right\}^{\perp}$ en sí mismo y la condición $|Z| u=0, u \in \operatorname{Im}|Z| \cap\left\{\psi, Z^{*} \psi^{\prime}\right\}^{\perp}$, implican que $u=|Z| u=0$, por consiguiente es inyectivo. Consecuentemente, el operador $Z q Z^{*}$ envía $\operatorname{Im} P_{3} \cap\left\{\psi^{\prime}, Z \psi\right\}^{\perp}$ en sí mismo y es inyectivo, pues las condiciones $Z q Z^{*} u=0$ y $u \in \operatorname{Im} P_{3} \cap\left\{\psi^{\prime}, Z \psi\right\}^{\perp}$, implican que $0=Z q Z^{*} u=Z|Z| Z^{*} u=Z Z^{*} Z Z^{*} u=P_{3} u=u$. Además, $Z q Z^{*}$ es una proyección ya que $Z q Z^{*} Z q Z^{*}=Z q|Z| q Z^{*}=Z q Z^{*}$ y para cualquier $u \in P_{3}(\mathrm{~h})=Z Z^{*}(\mathrm{~h})$ se tiene que $Z q Z^{*} u=Z q Z^{*} Z Z^{*} u=Z q|Z| Z^{*} u=$ $Z Z^{*} Z Z^{*} u=u$. Por lo tanto, coincide con la proyección ortogonal sobre $\operatorname{ImP}_{3} \cap$ $\left\{\psi^{\prime}, Z \psi\right\}^{\perp}$. Consecuentemente, por el inciso $(i v)$ del lema 3.3 se concluye que $P_{V \cap W_{D}^{\perp}}=q+Z q Z^{*}$. Finalmente, el resultado se sigue del teorema 3.5. Esto termina la prueba.

Corolario 3.4. El subespacio de recurrencia rápida del modelo de transporte cuántico contiene a $V \oplus \operatorname{span}\left\{e_{0}\right\}$, explícitamente,

$$
\mathcal{R} \supset\left\{e_{1}, \psi, \psi^{\prime}, Z \psi, Z^{*} \psi^{\prime}\right\}^{\perp}=V \oplus \operatorname{span}\left\{e_{0}\right\} .
$$

Demostración. Por el teorema 3.5, cada estado invariante con la estructura (3.21) está soportado en un subespacio de $\mathcal{R}$. El mismo teorema y el inciso (ii) en la proposición 3.8 implican que existe un estado invariante con soporte igual a $V$, pues $\rho$ 
es fiel en la subálgebra hereditaria $P_{W_{D}^{\perp} \cap V} B(\mathrm{~h}) P_{W_{D}^{\perp} \cap V}$. De las proposiciones 3.5 . 3.6, 3.7 y el corolario 3.1 se sigue el resultado. Esto termina la prueba.

Observación 3.16. Sean $\mathbf{k}_{1}=\operatorname{Im}|Z| \cap\left\{\psi, Z^{*} \psi^{\prime}\right\}^{\perp} y \mathbf{k}_{2}=\operatorname{Im} P_{3} \cap\left\{\psi^{\prime}, Z \psi\right\}^{\perp}$. Dado que la restricción del operador de inferencia $Z: \mathrm{k}_{1} \mapsto \mathrm{k}_{2}$ es un isomorfismo isométrico entre dos espacios de Hilbert, la transformación $\sigma \mapsto Z \sigma Z^{*}$ es una parametrización de todos los estados en $\mathcal{B}\left(\mathrm{k}_{2}\right)$ en términos de estados $\sigma \in \mathcal{B}\left(\mathrm{k}_{1}\right)$. Por este hecho, la estructura del estado invariante $\tau_{W_{D}^{\perp}}$ esta profundamente relacionada con el operador de interferencia $Z$ y con el operador de transporte T. Sin embargo, la estructura de $\tau_{W_{D}^{\perp}}$ coincide con la estructura general de los estados invariantes de semigrupos cuänticos de Markov uniformemente continuos descritos en el teorema 4.3 en [17]. En efecto, definiendo isomorfismos $U_{i}: \mathrm{k}_{i} \mapsto \mathrm{k}_{i} \otimes \mathbb{C}$, $i=1,2$ mediante $U_{i} u_{i}=u_{i} \otimes$ e con e el vector base de $\mathbb{C}$, se obtiene que el isomorfismo $U=U_{1} \oplus U_{2}, U: \mathrm{k}_{1} \oplus \mathrm{k}_{2} \mapsto\left(\mathrm{k}_{1} \otimes \mathbb{C}\right) \oplus\left(\mathrm{k}_{2} \otimes \mathbb{C}\right)$ satisface

$$
U \tau_{W_{D}^{\perp}} U^{*}=\operatorname{tr}\left(q \tau_{W_{D}^{\perp}}\right) \sigma \otimes \mathbb{1}+\operatorname{tr}\left(Z q Z^{*} \tau_{W_{D}^{\perp}}\right) Z \sigma Z^{*} \otimes \mathbb{1},
$$

donde

$$
\begin{aligned}
\operatorname{tr}\left(q \tau_{W_{D}^{\perp}}\right) & =\frac{\Gamma_{R e,+, \omega_{2}}}{\Gamma_{R e,+, \omega_{2}}+\Gamma_{R e,-, \omega_{2}}} \operatorname{tr}(\sigma q)=\frac{\Gamma_{R e,+, \omega_{2}}}{\Gamma_{R e,+, \omega_{2}}+\Gamma_{R e,-, \omega_{2}}}, \\
\operatorname{tr}\left(Z q Z^{*} \tau_{W_{D}}\right) & =\frac{\Gamma_{R e,-, \omega_{2}}}{\Gamma_{R e,+, \omega_{2}}+\Gamma_{R e,-, \omega_{2}}} \operatorname{tr}\left(Z \sigma Z^{*} Z q Z^{*}\right)=\frac{\Gamma_{R e,-, \omega_{2}}}{\Gamma_{R e,+, \omega_{2}}+\Gamma_{R e,-\omega_{2}}} .
\end{aligned}
$$

Las relaciones anteriores son válidas debido al hecho de que $\sigma$ es un estado soportado en $V \cap \operatorname{Im}|Z|$ y $P_{V \cap W_{D}^{\perp}}=q+Z q Z^{*}$.

\subsubsection{Aproximación al equilibrio y dominios de atracción}

La subálgebra libre de decoherencia $\mathcal{N}(\mathcal{T})$ del QMS, $\mathcal{T}$, del modelo de transporte cuántico fue estudiada y caracterizada en [5]. Se demostró que $\mathcal{N}(\mathcal{T}) \subset$ $\mathcal{F}(\mathcal{T})$, donde $\mathcal{F}(\mathcal{T})$ es el subconjunto de puntos fijos de $\mathcal{T}$, consecuentemente, $\mathcal{N}(\mathcal{T})=\mathcal{F}(\mathcal{T})$, dado que la inclusión opuesta siempre es verdadera. Si existiera un estado invariante fiel en $\mathcal{B}(\mathrm{h})$, de un resultado de Frigerio y Verri [26, 27] se podría concluir que para cualquier estado normal $\eta \in \mathcal{B}(\mathrm{h})$ existe el límite $\tau=\lim _{t \rightarrow \infty} \mathcal{T}_{* t}(\eta)$. Desafortunadamente, como consecuencia del teorema $3.5 \mathrm{y}$ observación 3.1, cualquier estado invariante $\tau$ del modelo de transporte cuántico es singular. De hecho, $V^{\perp} \backslash\left\{e_{0}\right\}=\operatorname{span}\left\{e_{1}, \psi, \psi^{\prime}, Z \psi, Z^{*} \psi^{\prime}\right\} \subset \operatorname{ker}(\tau)$. Por consiguiente, para analizar la propiedad de aproximación al equilibrio y dominios de atracción, es necesario restringir el análisis a la evolución en las subálgebras hereditarias $\mathcal{A}_{0}=P_{0} \mathcal{B}(\mathrm{h}) P_{0}, \mathcal{A}_{W_{D}}=P_{W_{D}} \mathcal{B}(\mathrm{h}) P_{W_{D}}$ y $\mathcal{A}_{W_{D}^{\perp} \cap V}=P_{W_{D}^{\perp} \cap V} \mathcal{B}(\mathrm{h}) P_{W_{D}^{\perp} \cap V}$. 
Observación 3.17. La dinámica en las subálgebras hereditarias $\mathcal{A}_{0}=P_{0} \mathcal{B}(\mathrm{h}) P_{0}$ y $\mathcal{A}_{W_{D}}=P_{W_{D}} \mathcal{B}(\mathrm{h}) P_{W_{D}}$ es irrelevante o trivial. La dinámica en la subálgebra hereditaria $\mathcal{A}_{W_{\bar{D}}^{\perp} \cap V}=P_{W_{\bar{D}}^{\perp} \cap V} \mathcal{B}(\mathrm{h}) P_{W_{D}^{\perp} \cap V}$ es más interesante y significativa para analizar la propiedad de aproximación al equilibrio y dominios de atracción. Por lo tanto, nos restringiremos a estudiar únicamente esta subálgebra hereditaria y comenzamos con el resultado siguiente.

Lema 3.4. Sea $\mathcal{T}_{W_{D}^{\perp} \cap V, t}=P_{W_{D}^{\perp} \cap V} \mathcal{T}_{t} P_{W_{D}^{\perp} \cap V}$ el semigrupo hereditario actuando sobre la subálgebra $\mathcal{A}_{W_{D}^{\perp} \cap V}$. Entonces $\mathcal{N}\left(\mathcal{T}_{W_{D}^{\perp} \cap V}\right) \subset \mathcal{F}\left(\mathcal{T}_{W_{D}^{\perp} \cap V}\right)$.

Demostración. Sea $a \in \mathcal{A}_{W_{D}^{\perp} \cap V}$ un elemento autoadjunto, entonces $a$ esta soportado en un subespacio de $V$, significando que $\left\{e_{0}, e_{1}, \psi, \psi^{\prime}, Z \psi, Z^{*} \psi^{\prime}\right\} \subset \operatorname{ker}(a)$. Entonces $a$ conmuta con $P_{0}$ y con $P_{1}$. Además, si $a \in \mathcal{N}\left(\mathcal{T}_{W_{D}^{\perp} \cap V}\right)$ implica que $a \in \mathcal{C}_{0}^{\prime}$, dado que $\mathcal{N}\left(\mathcal{T}_{W_{D}^{\perp} \cap V}\right) \subset \mathcal{C}_{0}^{\prime}$ (ver teorema 3.2 en [18]), donde

$$
\begin{aligned}
\mathcal{C}_{0} & =\left\{D_{\omega_{l}}, D_{\omega_{l}}^{*}: 1 \leq l \leq 3\right\} \\
& =\left\{|\psi\rangle\left\langle e_{1}|,| e_{1}\right\rangle\left\langle\psi\left|, Z, Z^{*},\right| \psi^{\prime}\right\rangle\left\langle e_{0}|,| e_{0}\right\rangle\left\langle\psi^{\prime}\right|\right\}
\end{aligned}
$$

consiste de los operadores de Kraus del modelo junto con sus adjuntos, restringidos a la subálgebra $\mathcal{A}_{W_{D}^{\perp} \cap V}$. En particular, $a$ conmuta con $Z$ y con $Z^{*}$, por lo tanto $a(\operatorname{ker}|Z|) \subset \operatorname{ker}|Z|$ y $a(\operatorname{Im}|Z|) \subset \operatorname{Im}|Z|$. Entonces, $a$ conmuta con $P_{2}=$ $\operatorname{Im}|Z| \oplus \operatorname{ker}|Z|$, y consecuentemente, conmuta con $P_{3}=I-P_{0}-P_{1}-P_{2}$. En conclusión, $a$ conmuta con $H$.

Por otra parte, la condición $\left\{e_{0}, e_{1}, \psi, \psi^{\prime}, Z \psi, Z^{*} \psi^{\prime}\right\} \subset \operatorname{ker}(a)$ implica que $a \in \operatorname{ker} \mathcal{L}_{\omega_{1}} \cap \operatorname{ker} \mathcal{L}_{\omega_{3}}$. Además, dado que $a$ conmuta con $P_{3}$ y con $|Z|$ se obtiene que $\left[H_{\omega_{2}}, a\right]=0$. La conmutación de $a \operatorname{con} Z$ y $Z^{*}$ implica que $a \in \operatorname{ker} \mathcal{L}_{\omega_{2}}$. Por lo tanto, $a$ es un punto fijo de $\mathcal{T}_{W_{D}^{\perp} \cap V}$. Considerando que cualquier operador es una combinación lineal de cuatro elementos autoadjuntos, el mismo resultado es válido para cualquier elemento $a \in \mathcal{A}_{W_{D}^{\frac{1}{D}} \cap V}$. Esto termina la prueba.

Corolario 3.5. Para cualquier estado inicial $\eta \in \mathcal{A}_{W_{D}^{\perp} \cap V}$ existe un estado invariante $\eta_{\infty}=\lim _{t \rightarrow \infty} \mathcal{T}_{W_{D}^{\perp} \cap V, t}(\eta) \in \mathcal{A}_{W_{D}^{\perp} \cap V}$.

Demostración. Es consecuencia inmediata del lema 3.4 el inciso (ii) en la proposición 3.8 y del resultado de Frigerio y Verri previamente citado [26, 27].

A continuación, se da una descripción completa de la evolución de cualquier estado soportado en $W_{D}^{\perp} \cap V$. Para hacerlo, se comienza por describir explícitamente la evolución de los estados soportados ya sea en $\operatorname{Im}|Z| \cap\left\{\psi, Z^{*} \psi^{\prime}\right\}^{\perp}$ o en $\operatorname{Im} P_{3} \cap\left\{\psi^{\prime}, Z \psi\right\}^{\perp}$. 


\section{Lema 3.5.}

(i) Sea $\sigma$ un estado soportado en $\operatorname{Im}|Z| \cap\left\{\psi, Z^{*} \psi^{\prime}\right\}^{\perp}$. Entonces $\mathcal{T}_{* t}(\sigma)$ converge a

$$
\frac{\Gamma_{R e,+, \omega_{2}}}{\Gamma_{R e,+, \omega_{2}}+\Gamma_{R e,-, \omega_{2}}} \sigma+\frac{\Gamma_{R e,-, \omega_{2}}}{\Gamma_{R e,+, \omega_{2}}+\Gamma_{R e,-, \omega_{2}}} Z \sigma Z^{*}
$$

cuando $t \rightarrow \infty$.

(ii) Sea $\eta$ un estado soportado en $\operatorname{Im} P_{3} \cap\left\{\psi^{\prime}, Z \psi\right\}^{\perp}$. Entonces $\mathcal{T}_{* t}(\eta)$ converge a

$$
\frac{\Gamma_{R e,+, \omega_{2}}}{\Gamma_{R e,+, \omega_{2}}+\Gamma_{R e,-, \omega_{2}}} Z^{*} \eta Z+\frac{\Gamma_{R e,-, \omega_{2}}}{\Gamma_{R e,+, \omega_{2}}+\Gamma_{R e,-, \omega_{2}}} \eta
$$

cuando $t \rightarrow \infty$.

Demostración. Sea $\sigma$ un estado soportado en $\operatorname{Im}|Z| \cap\left\{\psi, Z^{*} \psi^{\prime}\right\}^{\perp}$. Mediante cálculos directos se verifica que

$$
\begin{aligned}
\mathcal{L}_{*}(\sigma) & =-a \sigma+a Z \sigma Z^{*} ; & a & =2(N-1) \Gamma_{R e,-, \omega_{2}}, \\
\mathcal{L}_{*}\left(Z \sigma Z^{*}\right) & =b \sigma-b Z \sigma Z^{*} ; & b & =2(N-1) \Gamma_{R e,+, \omega_{2}} .
\end{aligned}
$$

Ahora, para cualquier observable $x \in \mathcal{B}(\mathrm{h})$ se definen $x_{t}=\operatorname{tr}\left(x \mathcal{T}_{* t}(\sigma)\right) \mathrm{y}$ $y_{t}=\operatorname{tr}\left(x \mathcal{T}_{* t}\left(Z \sigma Z^{*}\right)\right)$. Posteriormente, derivando $x_{t}$ con respecto a $t$ y utilizando (3.27), se obtiene

$$
\begin{aligned}
\dot{x}_{t} & =\operatorname{tr}\left(\mathcal{T}_{t}(x) \mathcal{L}_{*}(\sigma)\right) \\
& =\operatorname{tr}\left(\mathcal{T}_{t}(x)\left(-a \sigma+a Z \sigma Z^{*}\right)\right) \\
& =-a \operatorname{tr}\left(\mathcal{T}_{t}(x) \sigma\right)+a \operatorname{tr}\left(\mathcal{T}_{t}(x) Z \sigma Z^{*}\right) \\
& =-a \operatorname{tr}\left(x \mathcal{T}_{* t}(\sigma)\right)+a \operatorname{tr}\left(x \mathcal{T}_{* t}\left(Z \sigma Z^{*}\right)\right) \\
& =-a x_{t}+a y_{t} .
\end{aligned}
$$

De forma similar, se calcula la derivada de $y_{t}$ con respecto a $t$. Por consiguiente,

$$
\begin{aligned}
& \dot{x}_{t}=-a x_{t}+a y_{t} \\
& \dot{y}_{t}=b x_{t}-b y_{t}
\end{aligned}
$$

con $a=2(N-1) \Gamma_{R e,-, \omega_{2}}$ y $b=2(N-1) \Gamma_{R e,+, \omega_{2}}$. Por lo tanto, $\left(x_{t}, y_{t}\right)$ es una caminata aleatoria clásica con la matriz $Q$,

$$
Q=\left(\begin{array}{cc}
-a & a \\
b & -b
\end{array}\right)
$$


y distribución de probabilidad estacionaria $\pi=\left(\frac{b}{a+b}, \frac{a}{a+b}\right)$. Resolviendo el sistema (3.28) se obtiene,

$$
x_{t}=\operatorname{tr}\left(x\left(\frac{b+a e^{-t(a+b)}}{a+b} \sigma+\frac{a-a e^{-t(a+b)}}{a+b} Z \sigma Z^{*}\right)\right)
$$

lo cual implica que

$$
\mathcal{T}_{* t}(\sigma)=\frac{b+a e^{-t(a+b)}}{a+b} \sigma+\frac{a-a e^{-t(a+b)}}{a+b} Z \sigma Z^{*} \rightarrow \frac{b}{a+b} \sigma+\frac{a}{a+b} Z \sigma Z^{*}
$$

cuando $t \rightarrow \infty$.

Análogamente,

$$
y_{t}=\operatorname{tr}\left(x\left(\frac{b-b e^{-t(a+b)}}{a+b} \sigma+\frac{a+b e^{-t(a+b)}}{a+b} Z \sigma Z^{*}\right)\right),
$$

consecuentemente

$\mathcal{T}_{* t}\left(Z \sigma Z^{*}\right)=\frac{b-b e^{-t(a+b)}}{a+b} \sigma+\frac{a+b e^{-t(a+b)}}{a+b} Z \sigma Z^{*} \rightarrow \frac{b}{a+b} \sigma+\frac{a}{a+b} Z \sigma Z^{*}$

cuando $t \rightarrow \infty$. Esto prueba $(i)$.

Para probar $(i i)$ basta tomar $\sigma=Z^{*} \eta Z$ y aplicar $(i)$. Esto termina la prueba.

Teorema 3.7. Sea $\eta$ un estado inicial soportado en $W_{D}^{\perp} \cap V$. Entonces

$$
\lim _{t \rightarrow \infty} \mathcal{T}_{* t}(\eta)=\frac{\Gamma_{R e,+, \omega_{2}}}{\Gamma_{R e,+, \omega_{2}}+\Gamma_{R e,-, \omega_{2}}} \sigma+\frac{\Gamma_{R e,-, \omega_{2}}}{\Gamma_{R e,+, \omega_{2}}+\Gamma_{R e,-, \omega_{2}}} Z \sigma Z^{*}
$$

$\operatorname{con} \sigma=\frac{1}{\operatorname{tr}(q \eta)} q \eta q+\frac{1}{\operatorname{tr}\left(P_{3} \eta\right)} Z^{*} \eta Z$.

Demostración. Por el corolario 3.5. existe un estado invariante

$$
\eta_{\infty}=\frac{\Gamma_{R e,+, \omega_{2}}}{\Gamma_{R e,+, \omega_{2}}+\Gamma_{R e,-, \omega_{2}}} \sigma+\frac{\Gamma_{R e,-, \omega_{2}}}{\Gamma_{R e,+, \omega_{2}}+\Gamma_{R e,-, \omega_{2}}} Z \sigma Z^{*}
$$

donde $\sigma$ es un estado soportado en $\operatorname{Im}|Z| \cap\left\{\psi, Z^{*} \psi^{\prime}\right\}^{\perp}$, tal que $\lim _{t \rightarrow \infty} \mathcal{T}_{* t}(\eta)=$ $\eta_{\infty}$.

Ahora, del inciso $(i i)$ en la proposición 3.8 , cada estado inicial $\eta$ soportado en $W_{D}^{\perp} \cap V$ puede escribirse en la forma

$$
\eta=\left(q+Z q Z^{*}\right) \eta\left(q+Z q Z^{*}\right)=q \eta q+Z q Z^{*} \eta Z q Z^{*}+Z q Z^{*} \eta q+q \eta Z q Z^{*}
$$


El estado $\frac{1}{t r(q \eta)} q \eta q$ esta soportado en $\operatorname{Im}|Z| \cap\left\{\psi, Z^{*} \psi^{\prime}\right\}^{\perp}$ y por el lema 3.5 , es conducido por el semigrupo al estado límite

$$
\frac{1}{\operatorname{tr}(q \eta)}\left(\frac{\Gamma_{R e,+, \omega_{2}}}{\Gamma_{R e,+, \omega_{2}}+\Gamma_{R e,-, \omega_{2}}} q \eta q+\frac{\Gamma_{R e,-, \omega_{2}}}{\Gamma_{R e,+, \omega_{2}}+\Gamma_{R e,-, \omega_{2}}} Z q \eta q Z^{*}\right)
$$

cuando $t \rightarrow \infty$. Análogamente, del mismo lema 3.5 , el estado $\frac{1}{\operatorname{tr}\left(P_{3} \eta\right)} Z q Z^{*} \eta Z q Z^{*}=$ $\frac{1}{\operatorname{tr}\left(P_{3} \eta\right)} P_{3} \eta P_{3}$ esta soportado en $\operatorname{Im} P_{3} \cap\left\{\psi^{\prime}, Z \psi\right\}^{\perp}$ y

$$
\begin{aligned}
\lim _{t \rightarrow \infty} \mathcal{T}_{* t}\left(\frac{1}{\operatorname{tr}\left(P_{3} \eta\right)} P_{3} \eta P_{3}\right) & =\frac{1}{\operatorname{tr}\left(P_{3} \eta\right)}\left(\frac{\Gamma_{R e,+, \omega_{2}}}{\Gamma_{R e,+, \omega_{2}}+\Gamma_{R e,-, \omega_{2}}} Z^{*} P_{3} \eta P_{3} Z\right. \\
& \left.+\frac{\Gamma_{R e,-, \omega_{2}}}{\Gamma_{R e,+, \omega_{2}}+\Gamma_{R e,-, \omega_{2}}} P_{3} \eta P_{3}\right) .
\end{aligned}
$$

Comparando 3.29) con (3.30) y 3.31, se concluye que

$$
\lim _{t \rightarrow \infty} \mathcal{T}_{* t}\left(q \eta Z q Z^{*}+Z q Z^{*} \eta q\right)=0,
$$

y

$$
\sigma=\frac{1}{\operatorname{tr}(q \eta)} q \eta q+\frac{1}{\operatorname{tr}\left(P_{3} \eta\right)} Z^{*} P_{3} \eta P_{3} Z
$$

consecuentemente,

$$
Z \sigma Z^{*}=\frac{1}{\operatorname{tr}(q \eta)} Z q \eta q Z^{*}+\frac{1}{\operatorname{tr}\left(P_{3} \eta\right)} P_{3} \eta P_{3} .
$$

Esto termina la prueba.

Corolario 3.6. El dominio de atracción del estado invariante

$$
\rho=\frac{\Gamma_{R e,+, \omega_{2}}}{\Gamma_{R e,+, \omega_{2}}+\Gamma_{R e,-, \omega_{2}}} \sigma+\frac{\Gamma_{R e,-, \omega_{2}}}{\Gamma_{R e,+, \omega_{2}}+\Gamma_{R e,-, \omega_{2}}} Z \sigma Z^{*},
$$

donde $\sigma$ es un estado soportado en $\operatorname{Im}|Z| \cap\left\{\psi, Z^{*} \psi^{\prime}\right\}^{\perp}$, consiste de todos aquellos estados iniciales $\eta$ tal que

$$
\sigma=\frac{1}{\operatorname{tr}(q \eta)} q \eta q+\frac{1}{\operatorname{tr}\left(P_{3} \eta\right)} Z^{*} P_{3} \eta P_{3} Z .
$$

Demostración. Es consecuencia inmediata del teorema 3.7 
Observación 3.18. (Transporte de estados) Del resultado del teorema 3.7 la probabilidad total de un estado inicial $\eta$ es redistribuida en el límite cuando $t \rightarrow \infty$; así que la probabilidad en la porción del estado final $\eta_{\infty}$ soportado en $\operatorname{Im}|Z| \cap$ $\left\{\psi, Z^{*} \psi^{\prime}\right\}^{\perp}$ es

$$
\operatorname{tr}\left(q \eta_{\infty}\right)=\frac{\Gamma_{R e,+, \omega_{2}}}{\Gamma_{R e,+, \omega_{2}}+\Gamma_{R e,-, \omega_{2}}}
$$

y la probabilidad en la porción del estado final $\eta_{\infty}$ soportado en $\operatorname{Im} P_{3} \cap\left\{\psi^{\prime}, Z \psi\right\}^{\perp}$ es

$$
\operatorname{tr}\left(P_{3} \eta_{\infty}\right)=\frac{\Gamma_{R e,-, \omega_{2}}}{\Gamma_{R e,+, \omega_{2}}+\Gamma_{R e,-, \omega_{2}}} .
$$

Sin embargo, ajustando los valores de los coeficientes $\Gamma$ 's, de modo que $\frac{\Gamma_{R e,+, \omega_{2}}}{\Gamma_{R e,-, \omega_{2}}}=$ $e^{-\beta\left(\omega_{2}\right) \omega_{2}} \rightarrow 0$ (equivalentemente $\beta\left(\omega_{2}\right) \rightarrow \infty$ ), se puede "transportar" cualquier estado inicial $\eta$ a un estado límite $\eta_{\infty}$ concentrado (pero no necesariamente soportado) en el subespacio $\operatorname{Im} P_{3} \cap\left\{\psi^{\prime}, Z \psi\right\}^{\perp}$. En particular, cualquier estado inicial soportado en $\operatorname{Im}|Z| \cap\left\{\psi, Z^{*} \psi^{\prime}\right\}^{\perp}$ puede ser transportado, cuando $t \rightarrow \infty$, a un estado límite concentrado en el subespacio $\operatorname{Im} P_{3} \cap\left\{\psi^{\prime}, Z \psi\right\}^{\perp}$ (ver figura 3.1). En este sentido, el modelo de transporte cuántico permite "transportar" la masa de probabilidad de cualquier estado inicial a un estado concentrado en $\operatorname{Im} P_{3} \cap\left\{\psi^{\prime}, Z \psi\right\}^{\perp}$.

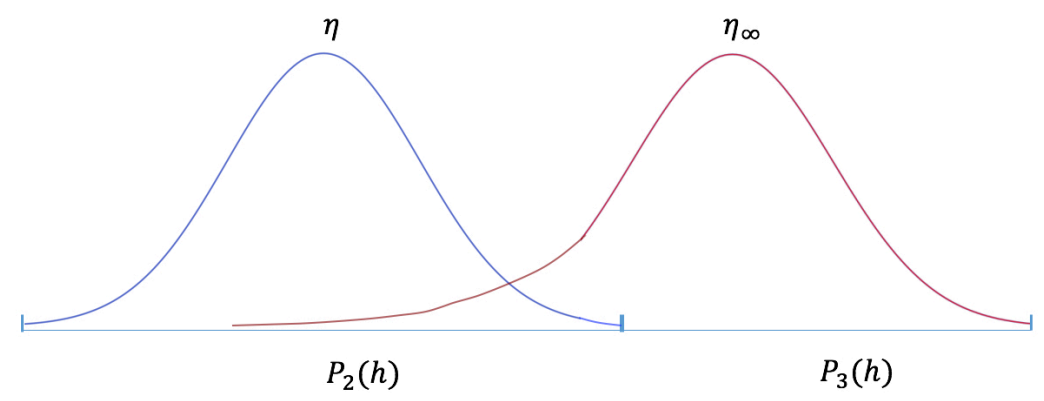

Figura 3.1: Transporte de masa de probabilidad

Definición 3.4. Sea $\rho$ un estado, la energía en el estado $\rho$ se define como $\operatorname{tr}\left(\rho H_{\text {eff }}\right)$.

Observación 3.19. (Ganancia de energía) A continuación, se verifica que si en $t=0$, el sistema se encuentra en cualquier estado inicial $\rho$ soportado en un subespacio de $\operatorname{Im}|Z| \cap\left\{\psi, Z^{*} \psi^{\prime}\right\}^{\perp}$, entonces, cuando $t \rightarrow \infty$ la energía del sistema 
crece y la ganancia de energía durante el proceso está dada por

$$
\operatorname{tr}\left(\rho_{\infty} H_{e f f}\right)-\operatorname{tr}\left(\rho H_{e f f}\right)=(N-1) \frac{\Gamma_{R e,-, \omega_{2}}}{\Gamma_{R e,+, \omega_{2}}+\Gamma_{R e,-, \omega_{2}}}\left(\Gamma_{I m,+, \omega_{2}}+\Gamma_{I m,-, \omega_{2}}\right),
$$

la cual es independiente de $\rho$. En efecto, sea $\rho$ un estado inicial soportado en $\operatorname{Im}|Z| \cap\left\{\psi, Z^{*} \psi^{\prime}\right\}^{\perp}$. El lema 3.5 implica que

$$
\rho_{\infty}=\frac{\Gamma_{R e,+, \omega_{2}}}{\Gamma_{R e,+, \omega_{2}}+\Gamma_{R e,-, \omega_{2}}} \rho+\frac{\Gamma_{R e,-, \omega_{2}}}{\Gamma_{R e,+, \omega_{2}}+\Gamma_{R e,-, \omega_{2}}} Z \rho Z^{*} .
$$

De la forma explícita del Hamiltoniano efectivo (3.12) se verifica que

$$
\rho_{\infty} H_{e f f}=(N-1)\left(\frac{\Gamma_{R e,-, \omega_{2}} \cdot \Gamma_{I m,+, \omega_{2}}}{\Gamma_{R e,+, \omega_{2}}+\Gamma_{R e,-, \omega_{2}}} Z \rho Z^{*}-\frac{\Gamma_{R e,+, \omega_{2}} \cdot \Gamma_{I m,-, \omega_{2}}}{\Gamma_{R e,+, \omega_{2}}+\Gamma_{R e,-, \omega_{2}}} \rho|Z|\right)
$$

$y$

$$
\rho H_{e f f}=-(N-1) \Gamma_{I m,-, \omega_{2}} \rho|Z| .
$$

Consecuentemente,

$\rho_{\infty} H_{e f f}-\rho H_{e f f}=(N-1) \frac{\Gamma_{R e,-, \omega_{2}}}{\Gamma_{R e,+, \omega_{2}}+\Gamma_{R e,-, \omega_{2}}}\left(\Gamma_{I m,+, \omega_{2}} Z \rho Z^{*}+\Gamma_{I m,-, \omega_{2}} \rho|Z|\right)$,

calculando la traza se obtiene el resultado. Obsérvese que este comportamiento es una indicación de que los sistemas abiertos degenerados (con un Hamiltoniano de referencia degenerado), parecen ser apropiados para modelar efectivamente la transferencia de energía cuántica en la fotosíntesis (ver [43] y sus referencias).

Observación 3.20. Nótese que los resultados en el transporte de estados y en la ganancia de energía están en dependencia de los coeficientes $\Gamma_{R e, \pm, \omega_{l}}$ 's, lo cual es consecuencia de que el sistema es abierto. Asimismo, los coeficientes $\Gamma_{I m, \pm, \omega_{l}}$ 's, $\Gamma_{R e, \pm, \omega_{l}}$ 's en la ganancia de energía, muestran la interacción entre el sistema cerrado (descrito por el Hamiltoniano efectivo $H_{\text {eff }}$ ) y su entorno o medio ambiente.

Ejemplo 3.3. (Un estado invariante $\rho \notin\{H\}^{\prime}$ ) Fijemos $N=4$ y $M=2$ en el modelo de transporte cuántico. Entonces,

$$
\begin{aligned}
& D_{\omega_{1}}=\sqrt{6 \Gamma_{R e,-, \omega_{1}}}\left|T e_{5}\right\rangle\left\langle e_{1}\right|, \quad D_{\omega_{2}}=\sqrt{6 \Gamma_{R e,-\omega_{2}}} Z, \\
& D_{\omega_{3}}=\sqrt{6 \Gamma_{R e,-, \omega_{3}}}\left|e_{0}\right\rangle\left\langle T e_{3}\right| .
\end{aligned}
$$


Considere el Hamiltoniano efectivo $\Delta=\Delta_{\omega_{1}}+\Delta_{\omega_{2}}+\Delta_{\omega_{3}}$, donde

$$
\begin{aligned}
\Delta_{\omega_{1}} & =-3 \Gamma_{R e,-, \omega_{1}} P_{1} \\
\Delta_{\omega_{2}} & =3 \Gamma_{I m,+, \omega_{2}} P_{3} \\
\Delta_{\omega_{3}} & =-3 \Gamma_{I m,-, \omega_{3}}\left|T e_{3}\right\rangle\left\langle T e_{3}\right| .
\end{aligned}
$$

Mediante cálculos directos se verifica que

$$
\begin{aligned}
W_{D} & =\operatorname{span}\left\{e_{0}, e_{1}, \psi, \psi^{\prime}\right\}^{\perp} \cap \operatorname{ker} Z \cap \operatorname{ker} Z^{*} \\
& =\left\{u_{2}(0,0,1, \kappa,-(\kappa+1), 0,0): u_{2} \in \mathbb{C}\right\}
\end{aligned}
$$

con $\kappa=\frac{\exp \left(\frac{4 \pi i}{3}\right)-1}{1-\exp \left(\frac{2 \pi i}{3}\right)} \neq 0$. Además, el estado $\rho_{u}=|u\rangle\langle u|$ con $u \in W_{D}, \quad\|u\|=$ 1 , conmuta con $P_{1}, P_{3}$ y con $\left|T e_{3}\right\rangle\left\langle T e_{3}\right|$. Consecuentemente, $\left[\Delta, \rho_{u}\right]=0$. Esto demuestra que $\rho_{u}$ es invariante. Además, $W_{D}=\left(P_{2}-|Z|\right)(\mathrm{h})$ con $|Z|<P_{2}$, entonces $|Z| u \neq P_{2} u$. Finalmente,

$$
\begin{aligned}
P_{2}|u\rangle\langle u| P_{2} & =|u\rangle\langle u| \\
& =\left|\left(P_{2}-|Z|\right) v\right\rangle\left\langle\left(P_{2}-|Z|\right) v\right| \\
& =\left|P_{2} v-\right| Z|v\rangle\left\langle P_{2} v-|Z| v\right| \\
& =\left|P_{2} v-\right| Z|v\rangle\left\langle P_{2} v|-| P_{2} v-|Z| v\right\rangle\langle|Z| v| \\
& =\left|P_{2} v\right\rangle\left\langle P_{2} v|-||Z| v\right\rangle\left\langle P_{2} v|-| P_{2} v\right\rangle\langle|Z| v|+||Z| v\rangle\langle|Z| v|
\end{aligned}
$$

Consecuentemente, $\rho_{u}$ no conmuta con $H$, i.e., $\rho_{u} \notin\{H\}^{\prime}$. Por lo tanto, en general, $\{H\}^{\prime} \subsetneq \operatorname{Ann}(D)$. No obstante, dado que $\rho_{u}=|u\rangle\langle u|$ está soportado en $W_{D}$, del teorema 3.2 se implica que $\rho_{u} \in A n n(D)$. Obsérvese que $M=2<3=N-1$, mostrando un caso particular del corolario 3.3 



\section{Conclusiones y perspectivas}

La primera parte de este trabajo contribuye a completar el estudio de la estructura de los estados estacionarios de generadores de Markov del límite de acoplamiento débil en dimensiones bajas. En [1], los autores introdujeron esta clase de generadores de Markov y obtuvieron estados estacionarios con estructura matricial a bloques que dependen de los coeficientes $\Gamma_{ \pm, \omega}$ 's. Por otra parte, los estados estacionarios descritos en el teorema 3.11 de la sección 3.2 tienen forma matricial a bloques, pero estos estados no dependen de los coeficientes $\Gamma_{ \pm, \omega}$ 's. A partir de lo anterior y de los dos casos estudiados en el capítulo 2, donde se calculan explícitamente este tipo de estados en dimensiones bajas, se concluye que entre los estados estacionarios de generadores de Markov de WCLT tenemos: estados de Gibbs, estados localmente de Gibbs y estados en forma matricial a bloques dependientes (o independientes) de los coeficientes $\Gamma_{ \pm, \omega}$ 's (ver tabla A.1 en apéndice A).

Tomando en cuenta la información obtenida en dimensiones bajas, en el capítulo 3, teoremas 3.3 y 3.4, logramos describir la estructura de cualquier estado estacionario que conmuta con la proyección ortogonal sobre el subespacio libre de interacción $P_{W_{D}}$, como una combinación lineal convexa de un estado soportado en $W_{D}$ y otro soportado en $W_{D}^{\perp}$, y que pertenece al aniquilador de todos los operadores de Kraus, $\operatorname{Ann}(D)$. Además se demostró que $P_{W_{D}} \in\{H\}^{\prime}$. Mediante la aplicación de los resultados previos, se caracterizó la estructura de estados estacionarios del modelo de transporte cuántico estudiado en [28] en términos del operador de transición o transporte $Z$, concluyendo que los estados estacionarios son de balance detallado y por consiguiente pertenecen al $A n n(D)$, aunque no necesariamente pertenecen a $\{H\}^{\prime}$. Además, se estudiaron las proyecciones armónicas y subarmónicas, el subespacio de recurrencia rápida, la convergencia al equilibrio y los dominios de atracción de los estados estacionarios de este modelo, mostrando en qué sentido la evolución reducida del sistema pequeño exhibe el fenómeno de transporte cuántico y ganancia de energía . 
Profundizar en el estudio del modelo de transporte cuántico de [28], fue esencial y de suma importancia para comprender más acerca de los generadores de Markov de WCLT. Además, el análisis del modelo presenta un hilo conductor de cómo abordar la búsqueda de estados estacionarios. No obstante, aún queda trabajo por desarrollar, tanto para los generadores de Markov de WCLT así como para el modelo de transporte cuántico.

A continuación se enuncian algunas sugerencias de estudio y perspectivas:

(I) Con relación a generadores de Markov de WCLT

I.1.- Investigar si todos los estados estacionarios que conmutan con $P_{W_{D}}$ (equivalentemente, tienen la estructura (3.7)) pertenecen a $\operatorname{Ann}(D)$.

I.2.- Investigar si existen estados estacionarios que no conmuten con $P_{W_{D}} \mathrm{o}$ equivalentemente, existencia de estados estacionarios cuya estructura difiera de (3.7). En caso de existir estos estados estacionarios, es natural preguntarse si pertenecen o no a $\operatorname{Ann}(D)$.

I.3.- Investigar la relación del subespacio libre de interacción $W_{D}$ con la noción de decoherencia para los semigrupos cuánticos de Markov [7, 8, 11]. En particular, con el subespacio libre de decoherencia [4, 36, 37].

(II) Con relación al modelo de transporte cuántico.

II.1.- Profundizar en el estudio de la transferencia y ganancia de energía.

II.2.- Como consecuencia del teorema 3.7, estudiar la velocidad de convergencia hacia el estado invariante $\eta_{\infty}$.

II.3.- Investigar la posibilidad de realizar una generalización del modelo de transporte, en términos del operador de transporte $T$. Esto último en analogía con las notables propiedades que poseen el operador de interferencia $Z$ y su adjunto $Z^{*}$. 
Apéndice A

\section{Apéndice A}

\section{Clasificación de estados estacionarios en dimensiones bajas}

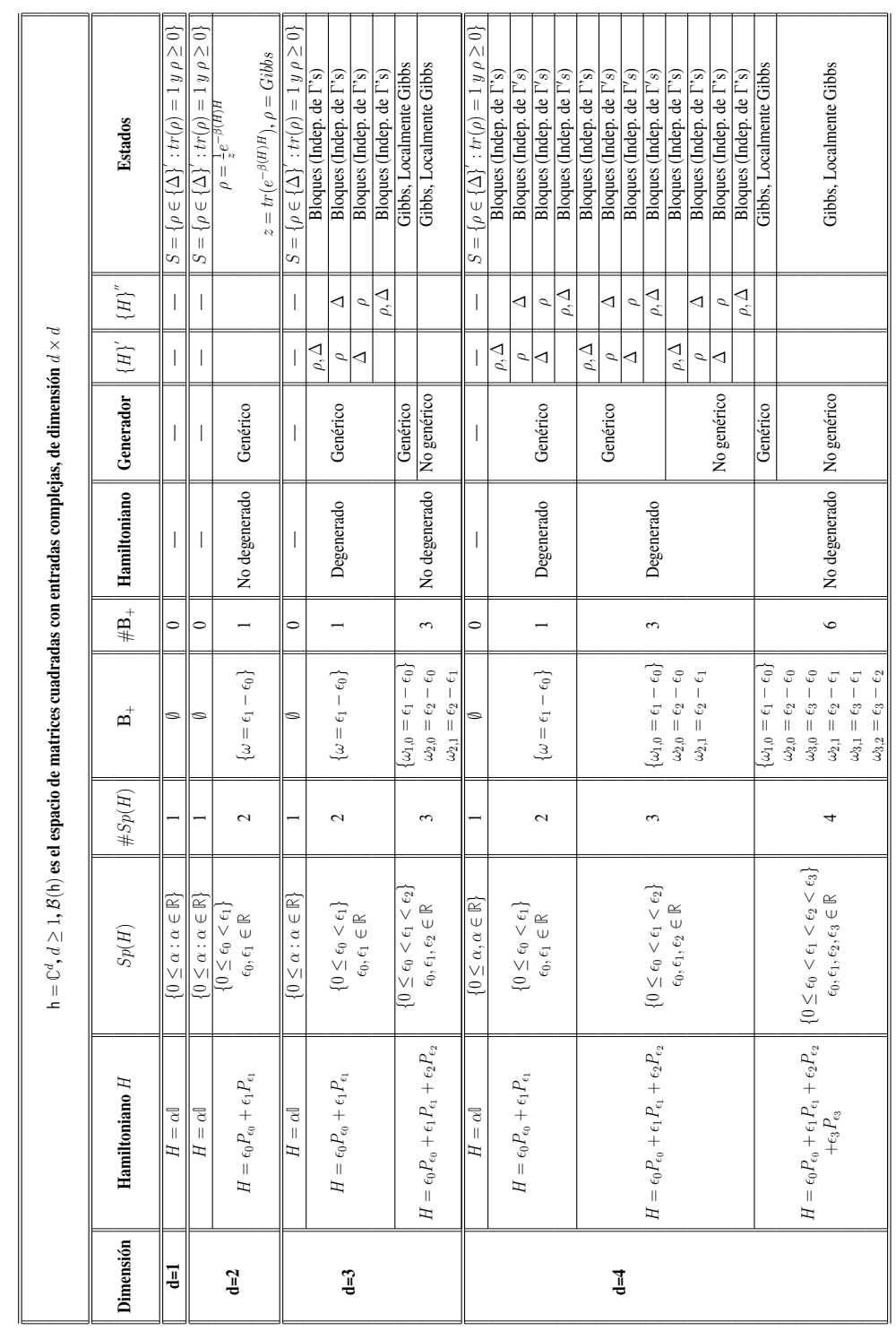

Tabla A.1: Clasificación de estados estacionarios en dimensiones bajas. 



\section{Referencias}

[1] Accardi, L., Fagnola, F. and Quezada, R. On three new principles in nonequilibrium statistical mechanics and Markov semigroups of weak coupling limit type, Infinite Dimensional Analysis, Quantum Probability and Related Topics 19, 02, (2016), 1650009.

[2] Accardi, L.,Kozyrev, S.V. and Pechen, A.N. Coherent quantum control of $\lambda$-atoms through the stochastic limit, Quantum Information and Computing. World Scientific, 2006, pp. 1-7.

[3] Accardi, L., Lu, Y. G. and Volovich, I. Quantum theory and its stochastic limit, Springer Science \& Business Media, 2013.

[4] Agredo, J., Fagnola, F. and Rebolledo, R. Decoherence free subspaces of a quantum Markov semigroup. Journal of Mathematical Physics 55,11 (2014), 112201.

[5] Agredo, J., García, J. C. and Quezada, R. Decoherence in an excitation energy transport model. Submitted to Proceedings of the 38th International Conference on Quantum Probability, Tokyo University of Science (2019).

[6] Aref'eva, I Y., Volovich, I. V. and Kozyrev, S. V. Stochastic limit method and interference in quantum many-particle systems. Theoretical and Mathematical Physics 183, 3 (2015), 782-799.

[7] Blanchard, P. and Olkiewicz, R. Decoherence induced transition from quantum to classical dynamics. Reviews in Mathematical Physics 15, 03 (2003), 217-243.

[8] Blanchard, P. and Olkiewicz, R. Decoherence as irreversible dynamical process in open quantum systems. In Open Quantum Systems III. Springer, 2006, pp. 117-159 
Referencias

[9] Bolaños-Servin, J.R. and Fagnola, F. On the structure of quantum Markov semigroups of weak coupling limit type. Journal of Physics: Conference Series 819, 1 (2017), 012003.

[10] Carbone, R., Fagnola, F., García, J.C. and Quezada, R. Spectral properties of the two-photon absorption and emission process, Journal of Mathematical Physics 49, 3 (2008), 032106.

[11] Carbone, R., Sasso, E. and Umanità, V. Decoherence for quantum Markov semi-groups on matrix algebras. In Annales Henri Poincaré (2013), vol. 14, Springer, pp. 681-697.

[12] Carbone, R., Sasso, E. and Umanità, V. Structure of generic quantum Markov semigroup. Infinite Dimensional Analysis, Quantum Probability and Related Topics 20, 02 (2017), 1750012.

[13] Carlen, E. A. and MaAs, J. Gradient flow and entropy inequalities for quantum Markov semigroups with detailed balance. Journal of Functional Analysis 273, 5 (2017) 1810-1869.

[14] Chebotarev, A. M. Lectures on quantum probability, vol. 4. Sociedad Matemática Mexicana México, 2000.

[15] Crismale, V. and Fidaleo, F. Exchangeable stochastic processes and symmetric states in quantum probability. Annali di Matematica Pura ed Applicata (1923-) 194, 4 (2015), 969-993.

[16] Cruz-de La Rosa, M. A. and Quezada, R. Non-equilibrium steady states of a Markov generator of weak coupling limit type modeling absorptionemission of $\mathrm{m}$ and $\mathrm{n}$ photons. Infinite Dimensional Analysis, Quantum Probability and Related Topics 19, 04 (2016), 1650023.

[17] Deschamps, J., Fagnola, F., Sasso, E. and Umanita, V. Structure of uniformly continuous quantum Markov semigroups. Reviews in Mathematical Physics 28, 01 (2016), 165003.

[18] Dhahri, A., Fagnola, F. and Rebolledo, R. The decoherence-free subalgebra of a quantum Markov semigroup with unbounded generator. Infinite Dimensional Analysis, Quantum Probability and Related Topics 13, 03 (2010), 413-433. 
Referencias

[19] Fagnola, F. Quantum Markov semigroups and quantum flows. Proyecciones 18, 3 (1999), 1-144

[20] Fagnola, F. and Quezada, R. A characterization of quantum Markov semigroups of weak coupling limit type, Infinite dimensional Analysis, Quantum probability and Related topics, 22, 2 (2019) 1950008 (10 pages), DOI: 10.1142/S0219025719500085

[21] Fagnola, F. and Quezada, R. Two-photon absorption and emission process. Infinite dimensional Analysis, Quantum probability and Related topics 8, 04 (2005), 573-591.

[22] Fagnola, F. and Rebolledo, R. Subharmonic projections for a quantum Markov semigroup.Journal of Mathematical Physics 43, 2 (2002), 10741082.

[23] Fagnola, F. and Rebolledo, R. Entropy production and detailed balance for a class of quantum Markov semigroups.Open Systems \& Information Dynamics 22, 03 (2015), 1550013.

[24] Fagnola, F., and Rebolledo, R. Entropy production for quantum Markov semigroups. Communications in Mathematical Physics 335, 2 (2015), 547570 .

[25] Fagnola, F., and Umanita, V. Generators of detailed balance quantum Markov semigroups. Infinite Dimensional Analysis, Quantum Probability and Related Topics 10, 03 (2007), 335-363.

[26] Frigerio, A. Quantum dynamical semigroups and approach to equilibrium. Letters in Mathematical Physics 2, 2 (1977), 79-87.

[27] Frigerio, A., and Verri, M. Long-time asymptotic properties of dynamical semigroups on $\mathrm{W}^{*}$-algebras. Mathematische Zeitschrift 180, 3 (1982), 275286.

[28] Garcia, J., Gliouez, S., Guerrero-Poblete, F., and Quezada, R. Entangled and dark stationary states of excitation energy transport models in quantum many-particle systems and photosynthesis. Infinite Dimensional Analysis, Quantum Probability and Related Topics 21 (2018). 
Referencias

[29] García, J. C., and Guerrero-Poblete, F. Some non-equilibrium invariant states for the asymmetric exclusion qms at level one. Infinite Dimensional Analysis, Quantum Probability and Related Topics 18, 01 (2015), 1550007.

[30] Goldstein, J. A. Semigroups of linear operators and applications. Courier Dover Publications, 2017.

[31] Gorini, V., Kossakowski, A., and Sudarshan, E. C. G. Completely positive dynamical semigroups of n-level systems. Journal of Mathematical Physics 17, 5 (1976), 821-825.

[32] Horowitz, J. M., and Parrondo, J. M. Entropy production along nonequilibrium quantum jump trajectories. New Journal of Physics 15, 8 (2013), 085028.

[33] Jakšić, V., Pillet, C.-A., and Westrich, M. Entropic fluctuations of quantum dynamical semigroups. Journal of Statistical Physics 154, 1-2 (2014), $153-187$.

[34] Kozyrev, S., and Volovich, I. Dark states in quantum photosynthesis. In Trends in Biomathematics: Modeling, Optimization and Computational Problems. Springer, 2018, pp. 13-26.

[35] Kraus, K. General state changes in quantum theory. Annals of Physics 64, 2 (1971), 311-335.

[36] LidAr, D. A. Review of decoherence free subspaces, noiseless subsystems, and dynamical decoupling. arXiv preprint arXiv:1208.5791 (2012).

[37] Lidar, D. A., Chuang, I. L., and Whaley, K. B. Decoherence-free subspaces for quantum computation. Physical Review Letters 81, 12 (1998), 2594.

[38] Lindblad, G. On the generators of quantum dynamical semigroups. Communications in Mathematical Physics 48, 2 (1976), 119-130.

[39] Masanori, O., Takeyuki, H., and Yun, C. L. H. White noise analysis and quantum information, vol. 34. World Scientific, 2017.

[40] Parthasarathy, K. R. An introduction to quantum stochastic calculus, vol. 85. Birkhäuser, 2012. 
Referencias

[41] PAzy, A. Semigroups of linear operators and applications to partial differential equations, vol. 44. Springer Science \& Business Media, 2012.

[42] ReEd, M., and Simon B. Methods of modern mathematical physics, IV: Analysis of operators, 1978.

[43] Scholes, G. D., Fleming, G. R., Olaya-Castro, A., and Van Grondelle, R. Lessons from nature about solar light harvesting. Nature chemistry 3, 10 (2011), 763.

[44] Stevens, M. The Kadison-Singer Property. Springer, 2016. 
Estados estacionarios de generadores de Markov del tipo limite de acoplamiento débil y modelos de

transporte cuántico.

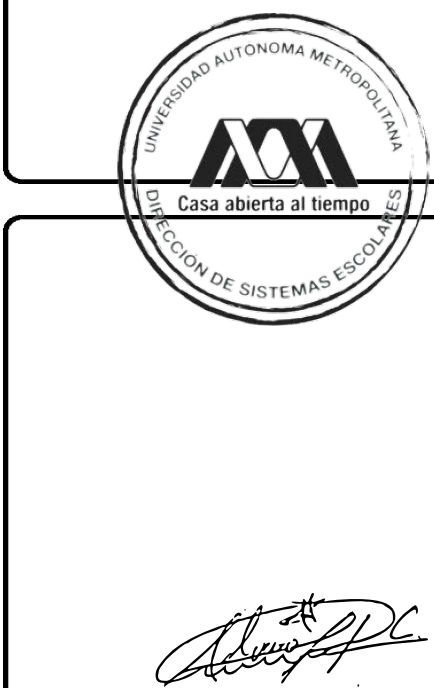

ALVARO HERNANDEZ CERVANTES ALUMNO

REVISO

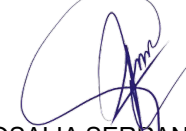

MTRA. ROSALIA SERIKANO DE LA PAZ DIRECTORA DE SISTENAS ESCOLARES

DIRECTOR DE LA DIVISIÓN DE CBI

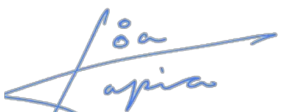

DR. JESUS ALBERTO OCHOA TAPIA
Con base en la Legislación de la Universidad Autónoma Metropolitana, en la Ciudad de México se presentaron a las 10:00 horas del día 28 del mes de enero del año 2021 POR VÍA REMOTA ELECTRÓNICA, los suscritos miembros del jurado designado por la Comisión del Posgrado:

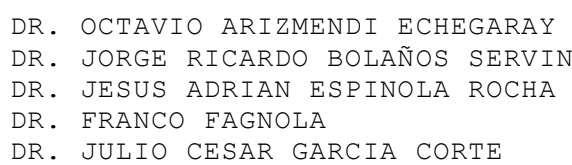

Bajo la presidencia del primero y con carácter de Secretario el último, se reunieron a la presentación de la Disertación Pública cuya denominación aparece al margen, para la obtención del grado de:

DOCTOR EN CIENCIAS (MATEMATICAS)

DE: ALVARo heRnAndeZ CERVAntes

y de acuerdo con el artículo 78 fracción IV del Reglamento de Estudios Superiores de la Universidad Autónoma Metropolitana, los miembros del jurado resolvieron:

$$
\text { APROBAR }
$$

Acto continuo, el presidente del jurado comunicó al interesado el resultado de la evaluación $y$, en caso aprobatorio, le fue tomada la protesta. 